\title{
Metalloradical Reactivity, Charge Transfer and Atom Abstractions in a T-shaped Iron(I) Complex
}

\author{
Jonas C. Ott, Hubert Wadepohl, and Lutz, H. Gade*
}

\begin{abstract}
Anorganisch-Chemisches Institut, Universität Heidelberg, Im Neuenheimer Feld 276, 69120 Heidelberg
*Correspondence to (L.H.G.) Fax: (+49) 6221-545609. E-mail: lutz.gade@uni-heidelberg.de
\end{abstract}

\section{Supporting Information}

S1 Experimental Procedures .............................................................................................2

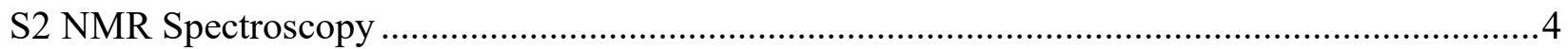

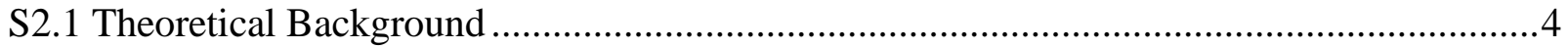

S2.2 Assignment of Paramagnetic NMR Spectra ..........................................................5

S2.3 General Acquisition Procedure .......................................................................... 9

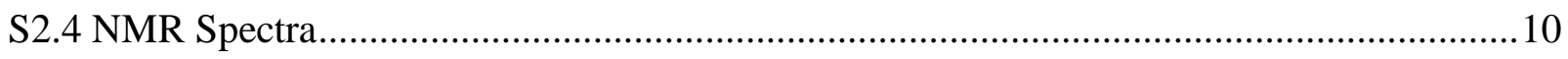

S2.5 Variable Temperature NMR Spectra ...............................................................22

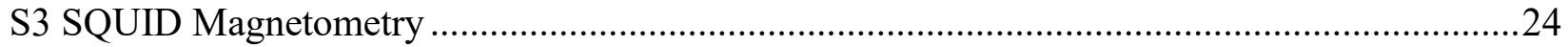

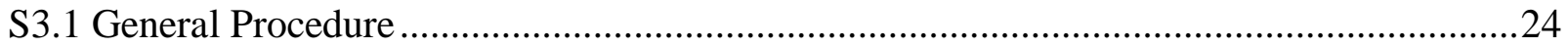

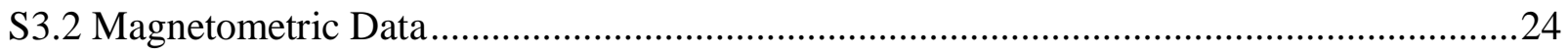

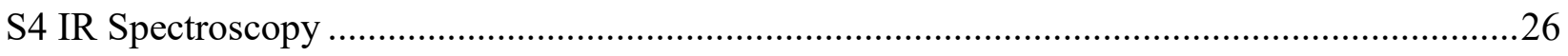

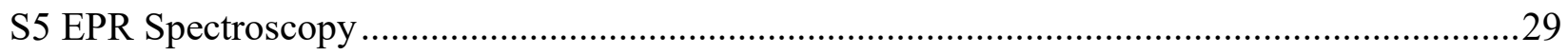

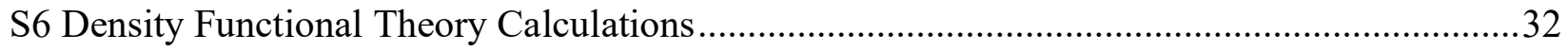

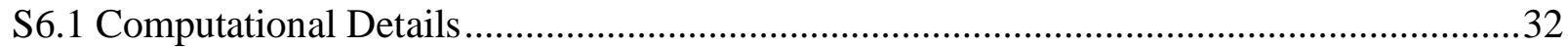

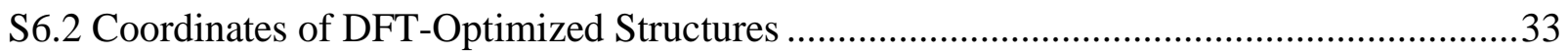

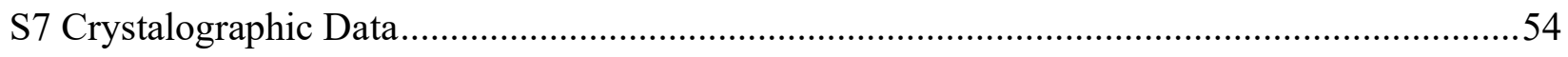

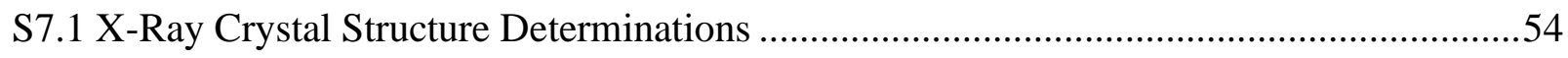

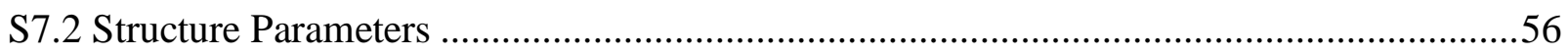

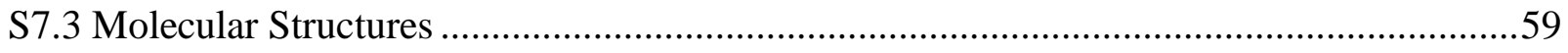

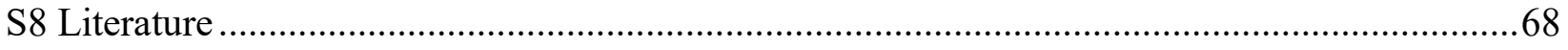




\section{S1 Experimental Procedures}

\section{Synthesis of $\left[{ }^{t \mathrm{Bu}}(\mathbf{P N P}) \mathrm{Fe}(\mathrm{CCPh})\right]$}

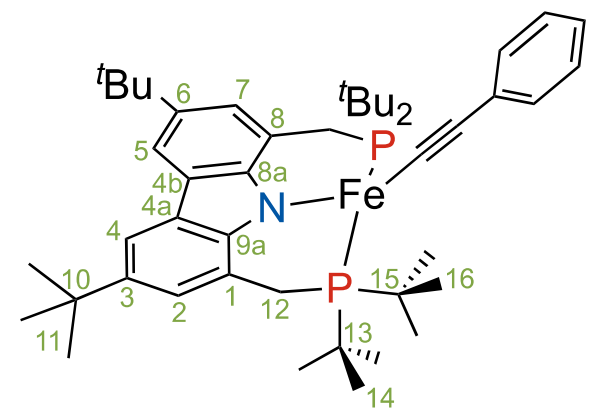

To a stirring solution of $\left[{ }^{\mathrm{tBu}}(\mathrm{PNP}) \mathrm{FeCl}\right]^{1}(50.0 \mathrm{mg}, 72.9 \mu \mathrm{mol}, 1.0$ eq. $)$ in THF $(1 \mathrm{ml})$ was dropwise added a solution of lithium phenylacetylide $(8.3 \mathrm{mg}, 80.2 \mu \mathrm{mol}, 1.1 \mathrm{eq}$.$) in THF (0.5 \mathrm{ml})$ and the reaction mixture was allowed to stir at ambient temperature overnight. The solvent was removed under reduced pressure, taken up in toluene and filtered through a pad of diatomaceous earth. All volatiles were removed in vacuo to yield $\left[{ }^{t \mathrm{Bu}}(\mathrm{PNP}) \mathrm{Fe}(\mathrm{CCPh})\right]$ as a dark red solid $(38.5 \mathrm{mg}$, $51.2 \mu \mathrm{mol}, 71 \%)$. Single crystals of the compound suitable for X-ray crystallography were obtained as dark red crystals by recrystallization of a saturated solution in $n$-pentane to $-40{ }^{\circ} \mathrm{C}$ overnight.

${ }^{1} \mathbf{H}$ NMR (600.13 MHz, $\left.\mathbf{C}_{6} \mathbf{D}_{6}, 295 \mathrm{~K}\right): \delta[\mathrm{ppm}]=40.8\left(\mathrm{~s}, 2 \mathrm{H}, H^{\mathrm{Carb} 4 / 5}\right), 31.5\left(\mathrm{~s}, 2 \mathrm{H}, H^{m-\mathrm{Ph}}\right), 8.9(\mathrm{~s}$, $\left.2 \mathrm{H}, H^{\mathrm{Carb} 2 / 7}\right), 4.8$ (bs, 36H, PC(CH3)3), 2.4 (s, $\left.18 \mathrm{H}, \mathrm{C}\left(\mathrm{CH}_{3}\right)_{3}\right),-14.5$ (s, 2H, $\left.H^{o-\mathrm{Ph}}\right),-19.6(\mathrm{~s}, 1 \mathrm{H}$, $H^{p-\mathrm{Ph}}$.

$\left\{{ }^{1} \mathbf{H}\right\}-{ }^{13} \mathbf{C}$ NMR (150.92 MHz, $\left.\mathbf{C}_{6} \mathbf{D}_{\mathbf{6}}, \mathbf{2 9 5} \mathbf{K}\right): \delta[\mathrm{ppm}]=929.1$ (s, 2C), 649.9 (s, 2C), 486.5 (s, 2C), 355.4 (s, 2C), 324.5 (s, 2C), 309.5 (s, 2C), 220.5 (s, 2C), 183.3 (s, 12C, $\left.\mathrm{PC}\left(\mathrm{CH}_{3}\right)_{3}\right), 96.9$ (s, 6C, $\left.\mathrm{C}\left(\mathrm{CH}_{3}\right)_{3}\right), 60.1$ (s, 2C), 58.6 (s, 2C), 8.1 (s, 2C), -37.1 (s, 2C).

Elemental Analysis: Calcd. for $\mathrm{C}_{46} \mathrm{H}_{67} \mathrm{FeNP}_{2}$ : C, 73.49; H, 8.98; N, 1.86. Found: C, 73.20; H, $8.88 ; \mathrm{N}, 1.71$.

Magnetic Susceptibility: $\mu_{\text {eff }}=4.6(2) \mu_{\text {в }}\left(\right.$ Evans, $\left.\mathrm{C}_{6} \mathrm{D}_{6}\right)$. 


\section{Synthesis of $\left[{ }^{t \mathrm{Bu}}(\mathrm{PNP}) \mathrm{Fe}(\mathrm{DCCPh})\right](4-\mathrm{D})$}

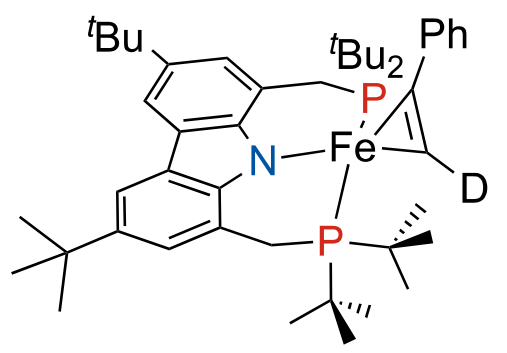

To a solution of 1 (10.0 mg, $15.4 \mu \mathrm{mol}, 1.0$ eq.) in toluene $(0.5 \mathrm{ml})$ was added phenylacetylene- $d_{1}(1.7 \mu$, $15.4 \mu \mathrm{mol}, 1.0$ eq.), followed by an immediate color change from yellow to dark red. The solution was allowed to stir for $30 \mathrm{~min}$ at ambient temperature, after which the solvent was removed under reduced pressure. The dark red residue was taken up in $n$-pentane and filtered through a pad of diatomaceous earth. All volatiles were removed in vacuo to yield $4-D$ as a dark red solid (6.3 mg, $8.4 \mu \mathrm{mol}, 55 \%)$.

${ }^{1}$ H NMR (399.89 MHz, C6D6, 295 K): $\delta[\mathrm{ppm}]=151.8$ (s, 2H, CHH), 134.6 (s, 2H, CHH), 24.4 $\left(\mathrm{s}, 2 \mathrm{H}, H^{\mathrm{Carb} 4 / 5}\right), 14.7\left(\mathrm{~s}, 18 \mathrm{H}, \mathrm{PC}\left(\mathrm{CH}_{3}\right)_{3}\right), 8.4\left(\mathrm{~s}, 2 \mathrm{H}, H^{\mathrm{Carb} 2 / 7}\right), 5.2\left(\mathrm{~s}, 2 \mathrm{H}, H^{m-\mathrm{Ph}}\right), 0.5(\mathrm{~s}, 18 \mathrm{H}$, $\left.\mathrm{C}\left(\mathrm{CH}_{3}\right)_{3}\right),-1.7\left(\mathrm{~s}, 18 \mathrm{H}, \mathrm{PC}\left(\mathrm{CH}_{3}\right)_{3}\right),-17.1\left(\mathrm{~s}, 2 \mathrm{H}, H^{o-\mathrm{Ph}}\right),-19.0\left(\mathrm{~s}, 1 \mathrm{H}, H^{p-\mathrm{Ph}}\right)$.

${ }^{2} \mathrm{H}$ NMR (61.31 MHz, $\left.\mathbf{C}_{6} \mathbf{H}_{6}, 295 \mathrm{~K}\right): \delta[\mathrm{ppm}]=-1250.7(\mathrm{~s}, 1 \mathrm{H}, D C C P h)$. 


\section{S2 NMR SPECTROSCOPY}

\section{S2.1 THEORETICAL BACKGROUND}

The observed chemical shift of a paramagnetic nucleus is equal to the sum of the orbital shift (which is equal to the diamagnetic contribution of the NMR shift) and the hyperfine shift (which is equal to the paramagnetic contribution of the NMR shift):

$$
\delta_{o b s}=\delta_{o r b}+\delta_{h f}
$$

The orbital shift may be calculated as the difference between the shielding constant of a reference nucleus (in most cases tetramethyl silane) and the shielding constant of the nucleus of interest, which can be calculated with the aid of DFT. As the orbital shift is a measure for the diamagnetic contribution, an alternative approach is to use a diamagnetic model compound, which can easily be assigned in the respective NMR spectra, in order to get sufficiently well approximated values for the diamagnetic shift of the related paramagnetic compound. On the other hand, the paramagnetic hyperfine contribution may be calculated by Eq. S2, where $S$ is the total electron spin of the system, $\mu_{\mathrm{B}}$ is the Bohr magneton, $k$ is the Boltzmann constant, $T$ is the temperature, $\gamma_{\mathrm{N}}$ is the gyromagnetic ratio of the nucleus of interest and $g$ and $\mathbf{A}^{\dagger}$ represent the matrices of the $g$ - and A-hyperfine tensors: ${ }^{2-4}$

$$
\delta_{h f}=\frac{S(S+1) \mu_{B}}{3 k T \gamma_{N}} \boldsymbol{g} \cdot \boldsymbol{A}^{\dagger}
$$

Eq. S2 is non-trivial as the matrix product of $\boldsymbol{g}$ and the transpose of $\boldsymbol{A}$ gives rise to a variety of nine terms, some of which are isotropic, some of which are anisotropic and one of them is both. However, for most compounds, the hyperfine shift can be simplified as the sum of the two most dominant contributions, which are the Fermi-contact $\left(\delta_{\text {fc }}\right)$ and the pseudocontact shift ( $\delta_{\text {psc }}$, Eq. S3): 4

$$
\delta_{h f} \approx \delta_{f c}+\delta_{p s c}
$$

While the pseudocontact shift is a result of the dipolar interaction of the nucleus of interest with the magnetic moment of the electrons (most often localized at the metal center) through space, the Fermi-contact shift is a result of the delocalization of unpaired spin density through covalent bonds from the metal center to the ligand atoms. This Fermi-contact contribution is therefore directly proportional to the residual spin density $\left(\rho_{\alpha \beta}\right)$ located at the nucleus of interest, which can be calculated with the aid of DFT, and is calculated by Eq. S4: ${ }^{4}$

$$
\delta_{f c S}=\frac{\mu_{0} \mu_{B}^{2} g_{e}^{2}}{9 k} \frac{(S+1)}{T} \rho_{\alpha \beta}
$$


For most $3 d$ metal complexes, the pseudocontact shift contribution is negligibly small when compared to the Fermi-contact contribution, which allows a determination of theoretical shift values $\left(\delta_{\text {calc }}\right)$ by Eq. S5 with sufficient accuracy:

$$
\delta_{\text {calc }}=\delta_{\text {orb }}+\delta_{f c}
$$

\section{S2.2 Assignment of PARAmagnetic NMR Spectra}

The calculation of the theoretical shifts was conducted with the aid of Equation S5. The unpaired spin densities $\rho_{\alpha \beta}$ were extracted from DFT calculations (vide infra) and together with Equation $\mathrm{S} 2$ exploited to calculate the Fermi-contact contributions. The orbital shifts were approximated by the experimentally obtained chemical shifts of the lithiated ligand ${ }^{t \mathrm{Bu}}(\mathrm{PNP}) \mathrm{Li} .{ }^{1}$ The numbering of the pincer ligand was conducted according to Scheme S1. A comparison of the calculated and experimentally observed chemical shifts is displayed in Tables S1 and S2.

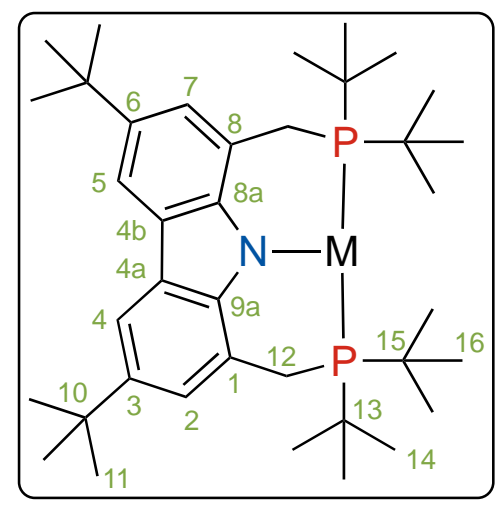

Scheme S1. Numbering scheme of the PNP pincer ligand.
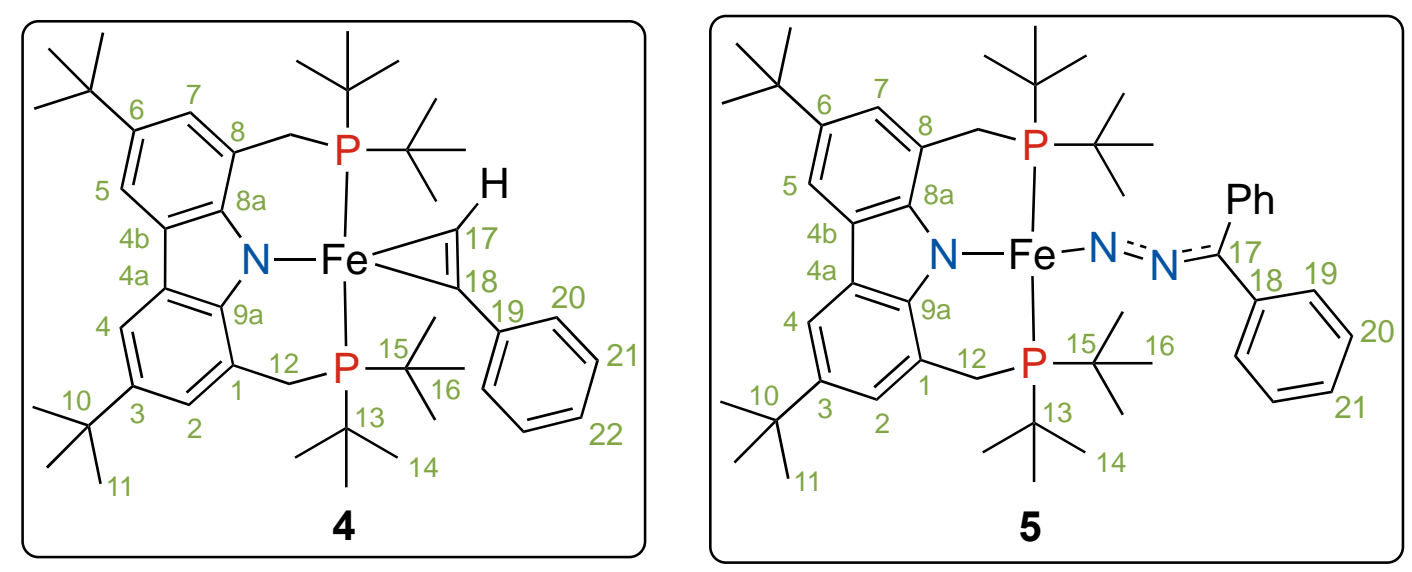

Scheme S2. Numbering scheme of complexes 4 and 5. 
Table S1. Assignment of ${ }^{1} \mathrm{H}$ and ${ }^{13} \mathrm{C}$ NMR Resonances of Complexes 4 and 5.

\begin{tabular}{|c|c|c|c|c|c|c|c|c|c|}
\hline \multirow{2}{*}{ \# } & \multicolumn{2}{|c|}{4} & \multicolumn{2}{|c|}{5} & \multirow{2}{*}{ \# } & \multicolumn{2}{|l|}{4} & \multicolumn{2}{|c|}{5} \\
\hline & $\delta_{\text {calc }}$ & $\delta_{\exp }^{[\mathrm{a}]}$ & $\delta_{\text {calc }}$ & $\delta_{\exp }^{\text {[a] }}$ & & $\delta_{\text {calc }}$ & $\delta_{\exp }^{[b]}$ & $\delta_{\text {calc }}$ & $\delta_{\exp }^{[b]}$ \\
\hline$H^{2 / 7}$ & 11.5 & 8.3 & 13.5 & 7.4 & $C^{1 / 8}$ & 246.3 & 234.2 & 283.2 & 266.6 \\
\hline$H^{4 / 5}$ & 29.1 & 24.5 & 37.1 & 28.9 & $C^{2 / 7}$ & 42.9 & 4.7 & 25.0 & 15.2 \\
\hline$H^{11}$ & 2.7 & 0.5 & 2.6 & 1.5 & $C^{3 / 6}$ & 288.4 & 329.0 & 309.3 & 301.3 \\
\hline$H^{12}$ & 93.8 & 135.0 & 116.7 & 135.7 & $C^{4 / 5}$ & 36.6 & -14.5 & 40.6 & -23.0 \\
\hline$H^{12^{\prime}}$ & 134.7 & 153.0 & 193.4 & 188.9 & $C^{4 \mathrm{a} / 4 \mathrm{~b}}$ & 413.1 & 421.5 & 595.3 & 459.4 \\
\hline$H^{14}$ & 8.0 & -1.7 & 9.8 & 3.9 & $C^{8 \mathrm{a} / 9 \mathrm{a}}$ & 1017.5 & n.o. & 888.1 & n.o. \\
\hline$H^{16}$ & 9.9 & 14.9 & 11.8 & 11.9 & $C^{10}$ & -12.0 & -28.8 & -20.0 & -36.3 \\
\hline$H^{17}$ & -1950.7 & -1267.9 & - & - & $C^{11}$ & 91.8 & 86.6 & 96.5 & 95.3 \\
\hline$H^{19}$ & - & - & -140.9 & -66.9 & $C^{12}$ & -61.9 & n.o. & 22.7 & n.o. \\
\hline$H^{20}$ & -16.9 & -17.1 & 44.8 & 31.0 & $C^{13}$ & 342.9 & n.o. & 742.2 & 510.5 \\
\hline$H^{21}$ & 5.0 & 5.1 & -99.5 & -60.1 & $C^{14}$ & 86.8 & 142.4 & 118.7 & 133.7 \\
\hline \multirow[t]{8}{*}{$H^{22}$} & -24.9 & -19.1 & - & - & $C^{15}$ & 431.6 & 445.6 & 719.3 & 484.4 \\
\hline & & & & & $C^{16}$ & 173.1 & 182.6 & 139.9 & 178.7 \\
\hline & & & & & $C^{17}$ & -14151.5 & n.o. & -5936.5 & n.o. \\
\hline & & & & & $C^{18}$ & -3355.1 & n.o. & -3423.8 & n.o. \\
\hline & & & & & $C^{19}$ & -3022.5 & n.o. & 715.6 & 706.5 \\
\hline & & & & & $C^{20}$ & 247.5 & n.o. & -410.7 & -186.6 \\
\hline & & & & & $C^{21}$ & -57.2 & 23.0 & 629.9 & 354.5 \\
\hline & & & & & $C^{22}$ & 281.4 & 268.9 & - & - \\
\hline
\end{tabular}

[a] $399.89 \mathrm{MHz}, \mathrm{C}_{6} \mathrm{D}_{6}, 295 \mathrm{~K}$. [b] 100.57 MHz, C6 $\mathrm{D}_{6}, 295 \mathrm{~K}$. 
Table S2. Assignment of ${ }^{1} \mathrm{H}$ and ${ }^{13} \mathrm{C}$ NMR Resonances of Complex 7.

\begin{tabular}{|c|c|c|c|c|c|}
\hline \multirow{2}{*}{ \# } & \multicolumn{2}{|c|}{7} & \multirow{2}{*}{ \# } & \multicolumn{2}{|c|}{7} \\
\hline & $\delta_{\text {calc }}$ & $\delta_{\exp }^{[a]}$ & & $\delta_{\text {calc }}$ & $\delta_{\exp }^{[b]}$ \\
\hline$H^{2 / 7}$ & 14.7 & 3.0 & $C^{1 / 8}$ & 330.9 & 277.4 \\
\hline$H^{4 / 5}$ & 44.6 & 35.6 & $C^{2 / 7}$ & -3.7 & 16.4 \\
\hline$H^{11}$ & 2.3 & -0.3 & $C^{3 / 6}$ & 324.8 & 315.6 \\
\hline$H^{12}$ & 170.5 & 155.1 & $C^{4 / 5}$ & 40.4 & 45.8 \\
\hline$H^{12}$ & 128.1 & 90.0 & $C^{4 \mathrm{a} / 4 \mathrm{~b}}$ & 833.5 & 764.6 \\
\hline \multirow[t]{7}{*}{$H^{14 / 16}$} & 15.6 & 19.9 & $C^{8 \mathrm{a} / 9 \mathrm{a}}$ & 644.3 & 642.8 \\
\hline & & & $C^{10}$ & -32.3 & -27.4 \\
\hline & & & $C^{11}$ & 97.3 & 84.0 \\
\hline & & & $C^{12}$ & 55.2 & -2.3 \\
\hline & & & $C^{13 / 15}$ & 861.8 & n.o. \\
\hline & & & $C^{14 / 16}$ & 189.5 & 179.8 \\
\hline & & & $C^{17}$ & 2602.5 & n.o. \\
\hline
\end{tabular}

[a] 600.13 MHz, $\mathrm{C}_{6} \mathrm{D}_{6}, 295 \mathrm{~K}$. [b] $150.92 \mathrm{MHz}, \mathrm{C}_{6} \mathrm{D}_{6}, 295 \mathrm{~K}$.

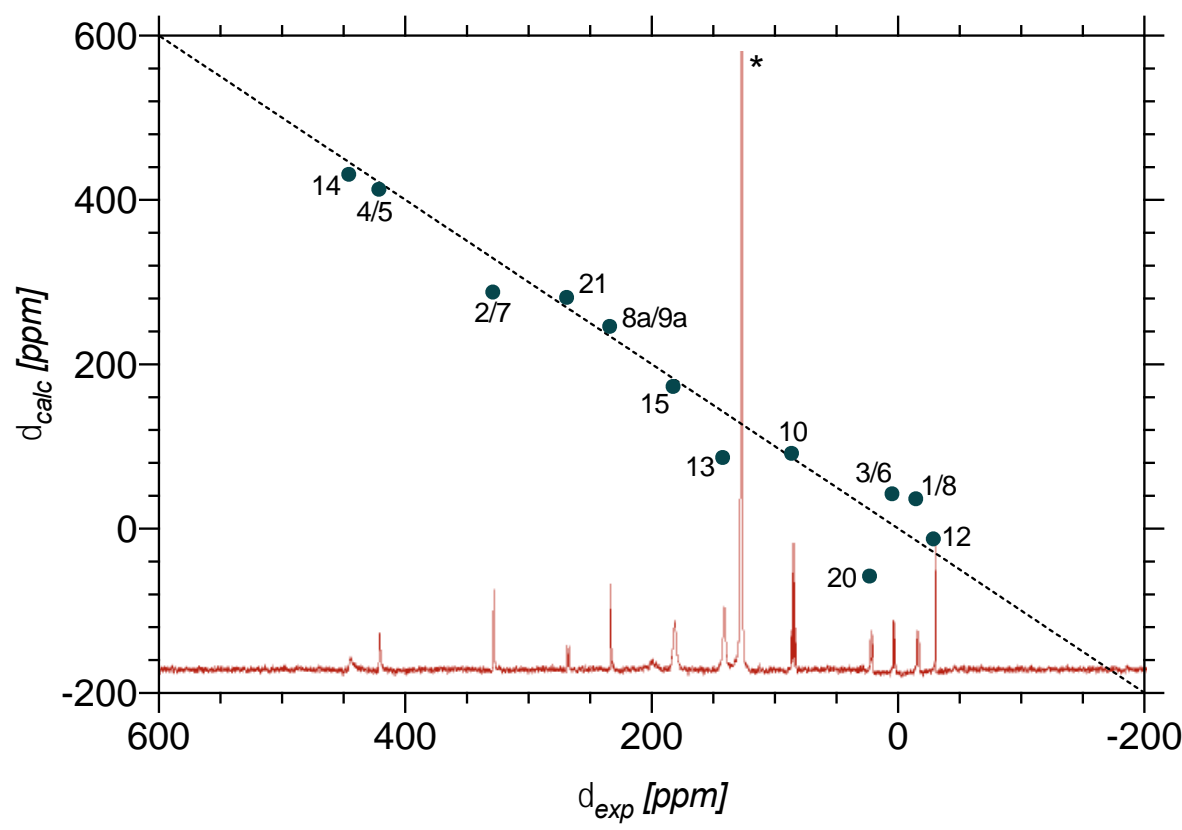

Figure S1. Plot of the correlation between experimental $\left(295 \mathrm{~K}, 100.57 \mathrm{MHz}, \mathrm{C}_{6} \mathrm{D}_{6}\right)$ and calculated $[\mathrm{B} 3 \mathrm{LYP} / \mathrm{def} 2 \mathrm{tzvp}]{ }^{13} \mathrm{C}$ NMR resonances of complex 4 . The dotted line represents a perfect correlation $(\mathrm{m}=$ $1.0, \mathrm{y}=0.0)$. The solvent resonance is indicated by an asterisk. 


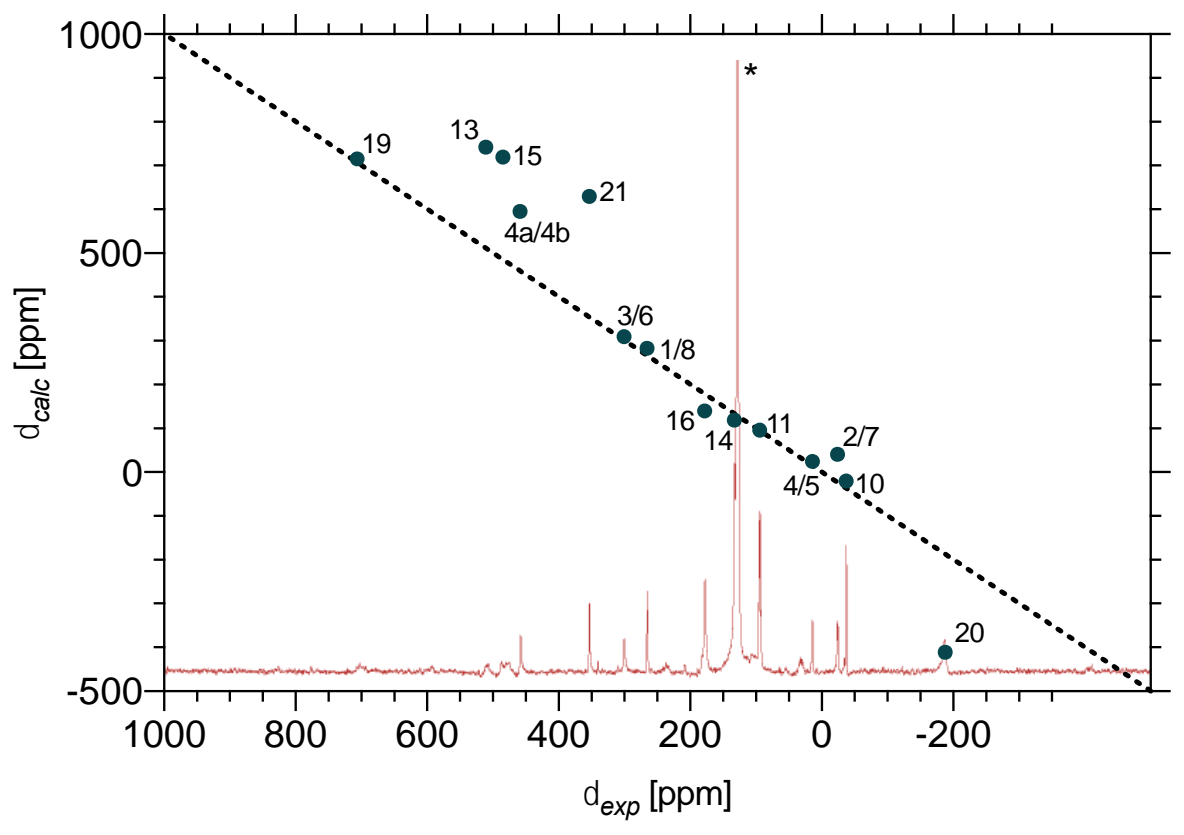

Figure S2. Plot of the correlation between experimental $\left(295 \mathrm{~K}, 100.57 \mathrm{MHz}, \mathrm{C}_{6} \mathrm{D}_{6}\right)$ and calculated $[\mathrm{B} 3 \mathrm{LYP} / 6-311 \mathrm{G}(\mathrm{d}, \mathrm{p})+\operatorname{def} 2 \operatorname{tzvp}(\mathrm{Fe}$ only $)]{ }^{13} \mathrm{C}$ NMR resonances of complex 5. The dotted line represents a perfect correlation $(\mathrm{m}=1.0, \mathrm{y}=0.0)$. The solvent resonance is indicated by an asterisk. 


\section{S2.3 General ACQuisition Procedure}

Unless stated otherwise, all spectra were recorded at a probe temperature of $295 \mathrm{~K}$. The used data acquisition parameters of the paramagnetic ${ }^{1} \mathrm{H}$ and ${ }^{13} \mathrm{C}$ NMR experiments are displayed in Tables S3 and S4. The NMR spectra were either recorded on a Bruker Avance III $600 \mathrm{MHz}$ spectrometer, equipped with a He-cooled Cryo probe, or on a Bruker Avance II $400 \mathrm{MHz}$ spectrometer. Furthermore, all temperature dependent data was recorded on the latter. The spectra were processed and analyzed with the Bruker NMR software Topspin 4.1.0 and printed without further manipulation.

Table S3. Acquisition Parameters for Paramagnetic ${ }^{1} \mathrm{H}$ and ${ }^{13} \mathrm{C}$ NMR Experiments at Bruker AVIII 600.

\begin{tabular}{lccc}
\hline Parameter & ${ }^{1} \mathrm{H}$ & ${ }^{13} \mathrm{C}$ & HETCOR \\
\hline Pulse program $($ Bruker $)$ & $\mathrm{zg}$ & $\mathrm{zg}$ & hxcoqf \\
$90^{\circ}$ pulse width $p 1[\mu \mathrm{s}]$ & 14.0 & 12.0 & $12.0\left({ }^{13} \mathrm{C}\right)$ \\
Pulse width used $\left[{ }^{\circ}\right] /[\mu \mathrm{s}]$ & $9 / 1.4$ & $60 / 8.0$ & $90 / 12.0\left({ }^{13} \mathrm{C}\right)$ \\
Sweep width $F 1[\mathrm{ppm}]$ & 1500 & 2000 & 300 \\
Sweep width $F 2[\mathrm{ppm}]$ & & & 200 \\
Acquisition data points $t d$ & $64 \mathrm{k}$ & $98 \mathrm{k}$ & $8 \mathrm{k} \times 128$ \\
Resulting acquisition time $a q[\mathrm{~s}]$ & 0.055 & 0.157 & $0.09 \mid 0.0005$ \\
Relaxation delay $d 1[\mathrm{~s}]$ & 0.01 & 0.05 & 0.05 \\
Preacquisition delay $d e[\mu \mathrm{s}]$ & 6.5 & 18.0 & 18.0 \\
Number of scans $n s$ & $4 \mathrm{k}$ & $64 \mathrm{k}$ & 128 \\
\hline
\end{tabular}

Table S4. Acquisition Parameters for Paramagnetic NMR Experiments at Bruker AVII 400.

\begin{tabular}{lcccc}
\hline Parameter & ${ }^{1} \mathrm{H}$ & ${ }^{2} \mathrm{H}$ & ${ }^{13} \mathrm{C}$ & HETCOR \\
\hline Pulse program $($ Bruker $)$ & $\mathrm{zg}$ & $\mathrm{zg} 2 \mathrm{H}$ & $\mathrm{zg}$ & hxcoqf \\
$90^{\circ}$ pulse width $p 1[\mu \mathrm{s}]$ & 14.0 & 76.0 & 10.0 & $10.0\left({ }^{13} \mathrm{C}\right)$ \\
Pulse width used $\left[{ }^{\circ}\right] /[\mu \mathrm{s}]$ & $30 / 4.7$ & $90 / 76.0$ & $60 / 6.7$ & $90 / 10.0\left({ }^{13} \mathrm{C}\right)$ \\
Sweep width $F 1[\mathrm{ppm}]$ & 800 & 600 & 2000 & 800 \\
Sweep width $F 2[\mathrm{ppm}]$ & & & & 150 \\
Acquisition data points $t d$ & $64 \mathrm{k}$ & $8 \mathrm{k}$ & $64 \mathrm{k}$ & $8 \mathrm{k} \times 64$ \\
Resulting acquisition time $a q[\mathrm{~s}]$ & 0.1 & 0.1 & 0.1 & $0.05 / 0.001$ \\
Relaxation delay $d 1[\mathrm{~s}]$ & 0.05 & 0.05 & 0.1 & 0.1 \\
Preacquisition delay $d e[\mu \mathrm{s}]$ & 6.5 & 20.0 & 6.5 & 6.5 \\
Number of scans $n s$ & $2 \mathrm{k}$ & $2 \mathrm{k}$ & $64 \mathrm{k}$ & 64 \\
\hline
\end{tabular}




\section{S2.4 NMR SPECTRA}

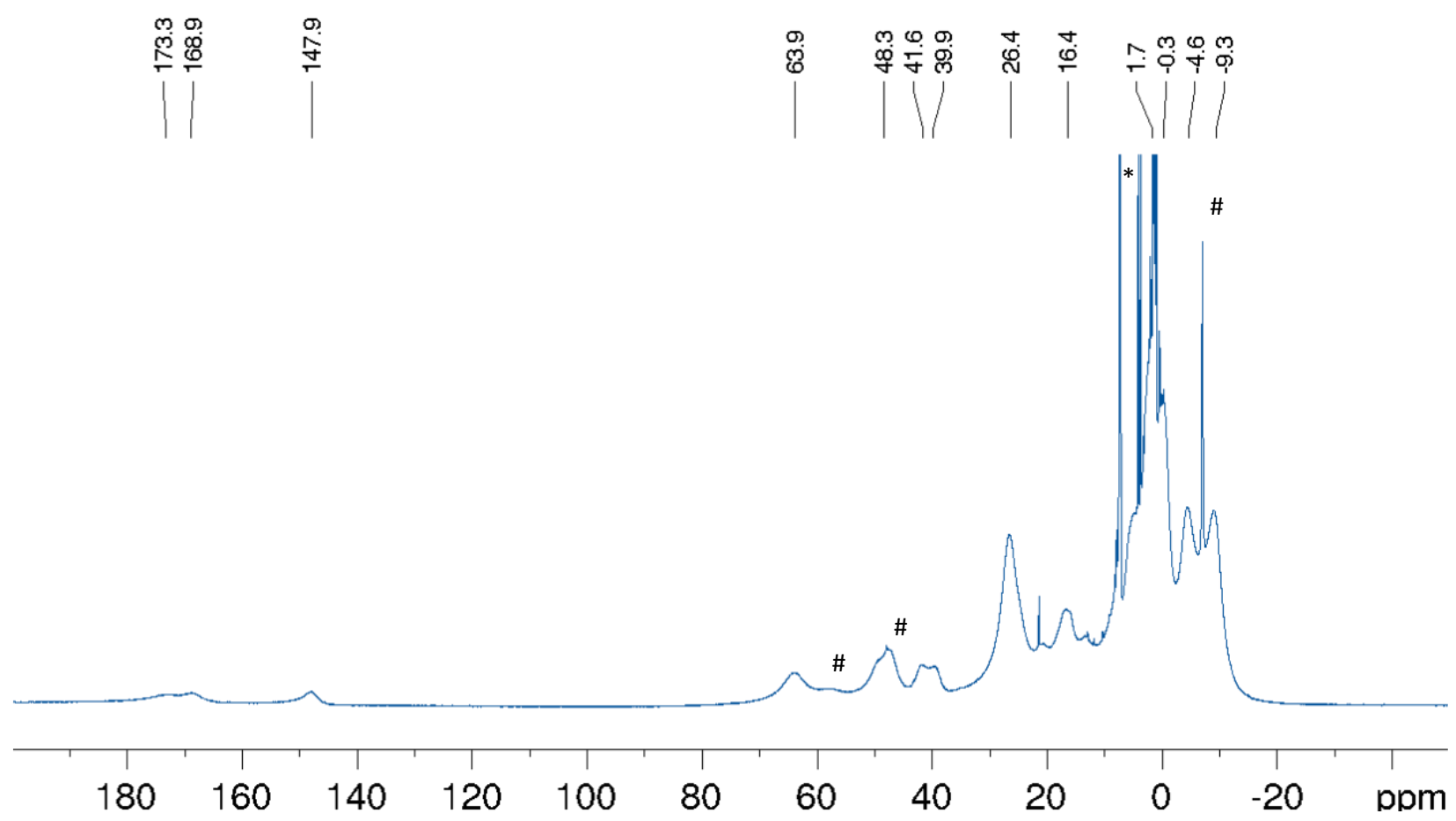

Figure S3. ${ }^{1} \mathrm{H}$ NMR spectrum $\left(399.89 \mathrm{MHz}, \mathrm{C}_{6} \mathrm{D}_{6}, 295 \mathrm{~K}\right.$ ) of complex 2 . The resonance of the solvent is indicated by an asterisk. The resonances of a minor unidentified paramagnetic impurity are hashed.

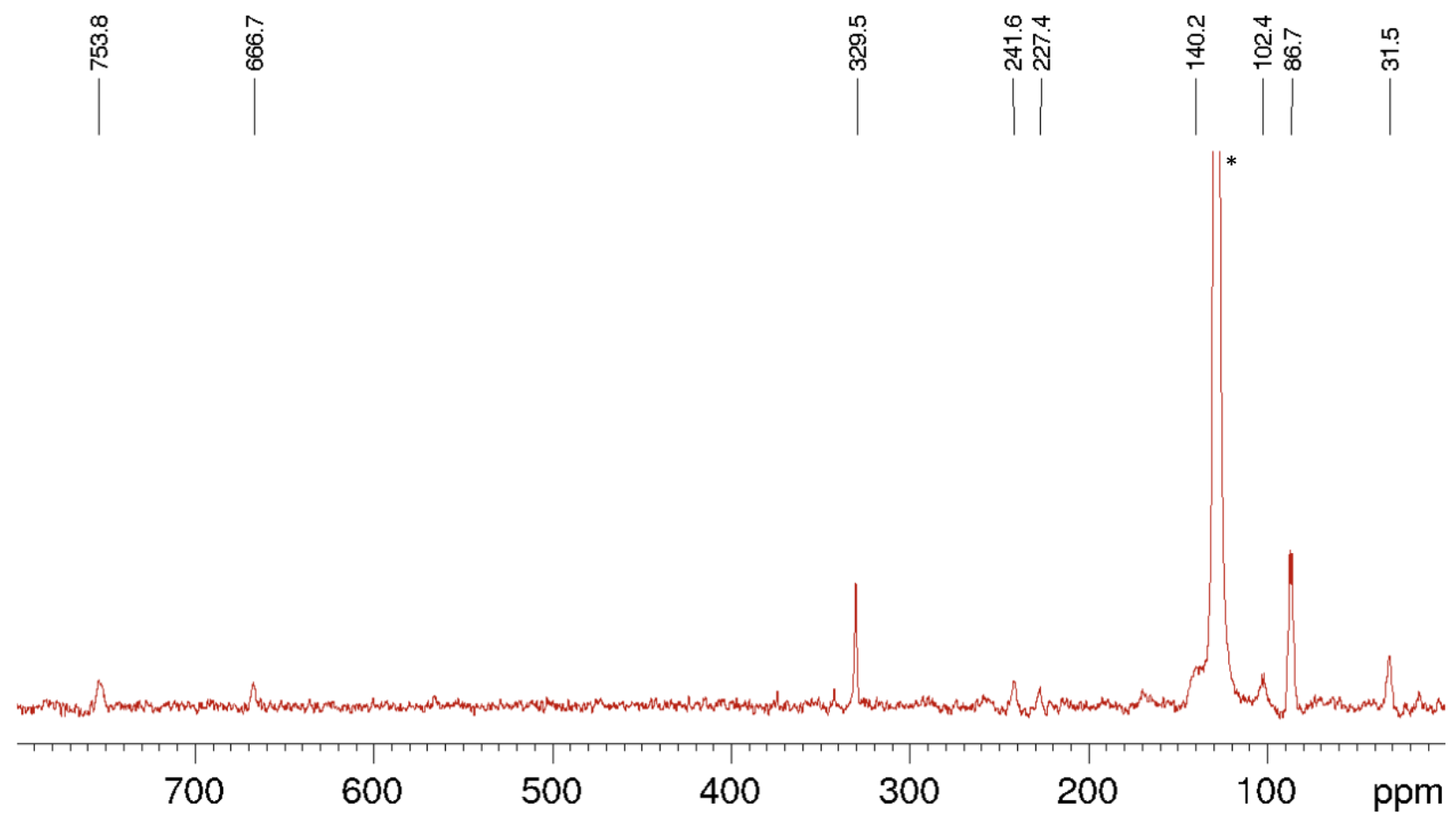

Figure S4. ${ }^{13} \mathrm{C}$ NMR spectrum $\left(100.61 \mathrm{MHz}, \mathrm{C}_{6} \mathrm{D}_{6}, 295 \mathrm{~K}\right)$ of complex 2 . The resonance of the solvent is indicated by an asterisk. 


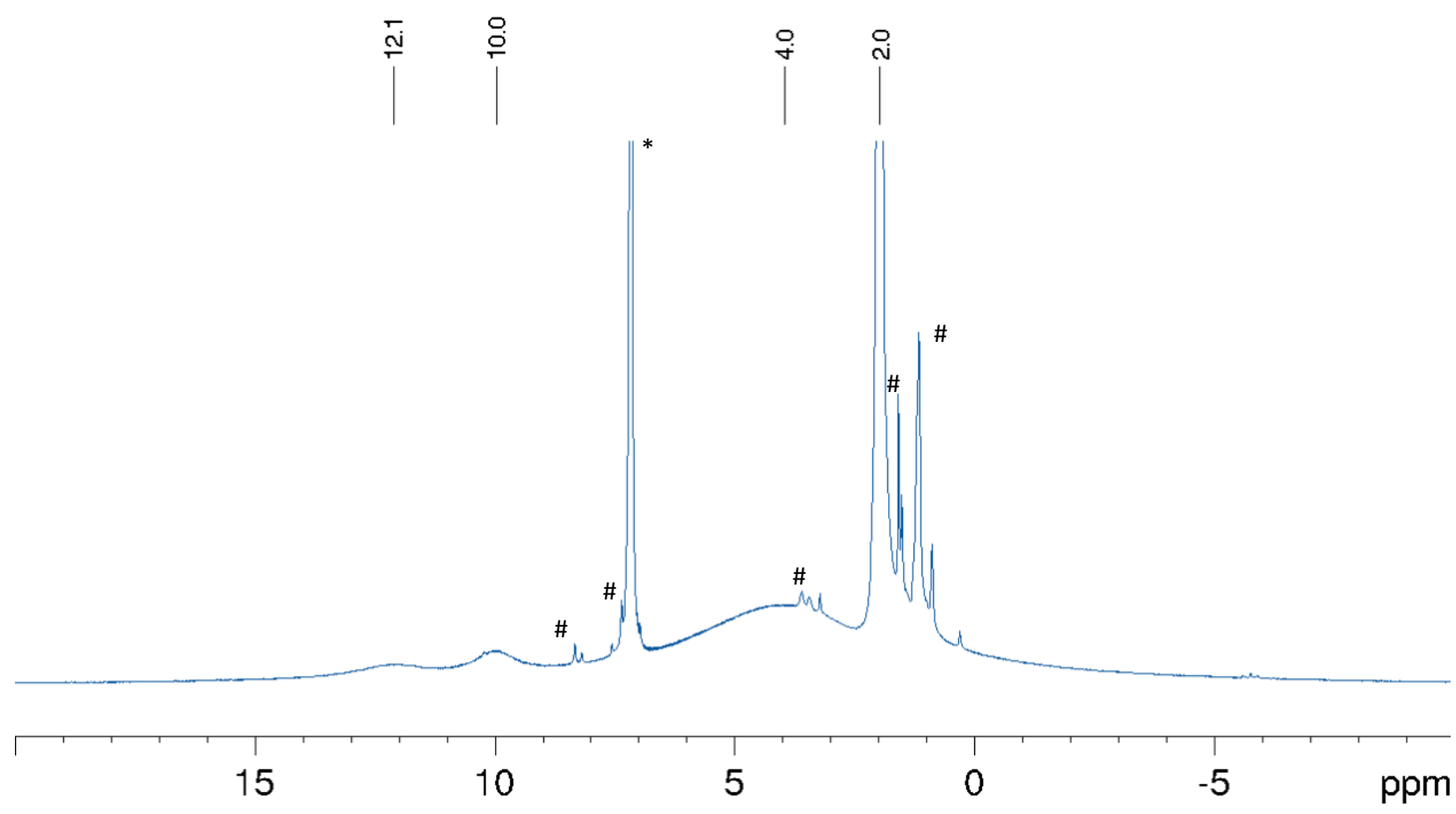

Figure S5. ${ }^{1} \mathrm{H}$ NMR spectrum $\left(399.89 \mathrm{MHz}, \mathrm{C}_{6} \mathrm{D}_{6}, 295 \mathrm{~K}\right)$ of complex 3. The resonance of the solvent is indicated by an asterisk. Diamagnetic resonances of the proteo ligand are hashed.
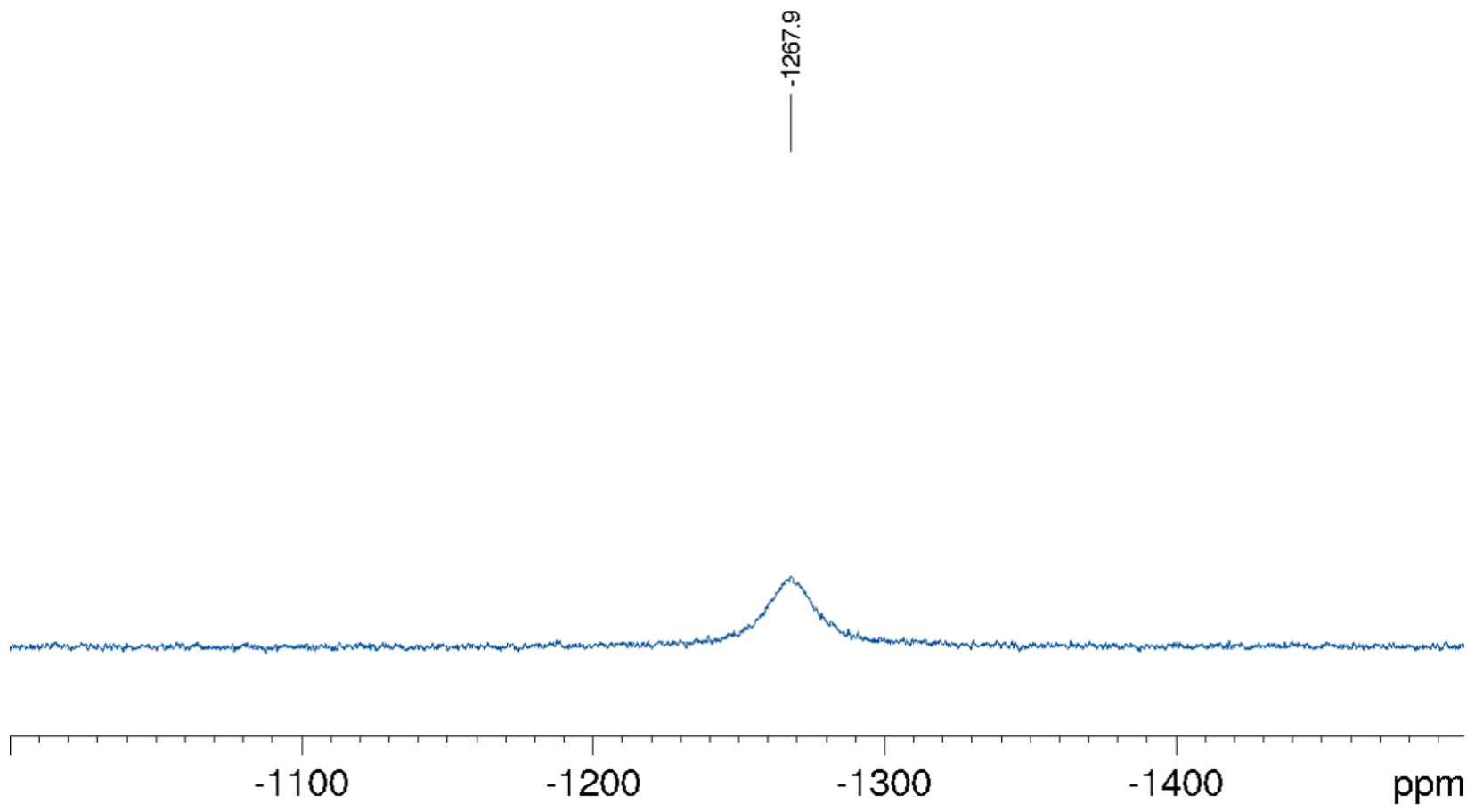

Figure S6. High-field ${ }^{1} \mathrm{H}$ NMR spectrum (399.89 MHz, C6 $\left.\mathrm{D}_{6}, 295 \mathrm{~K}\right)$ of complex 4. 

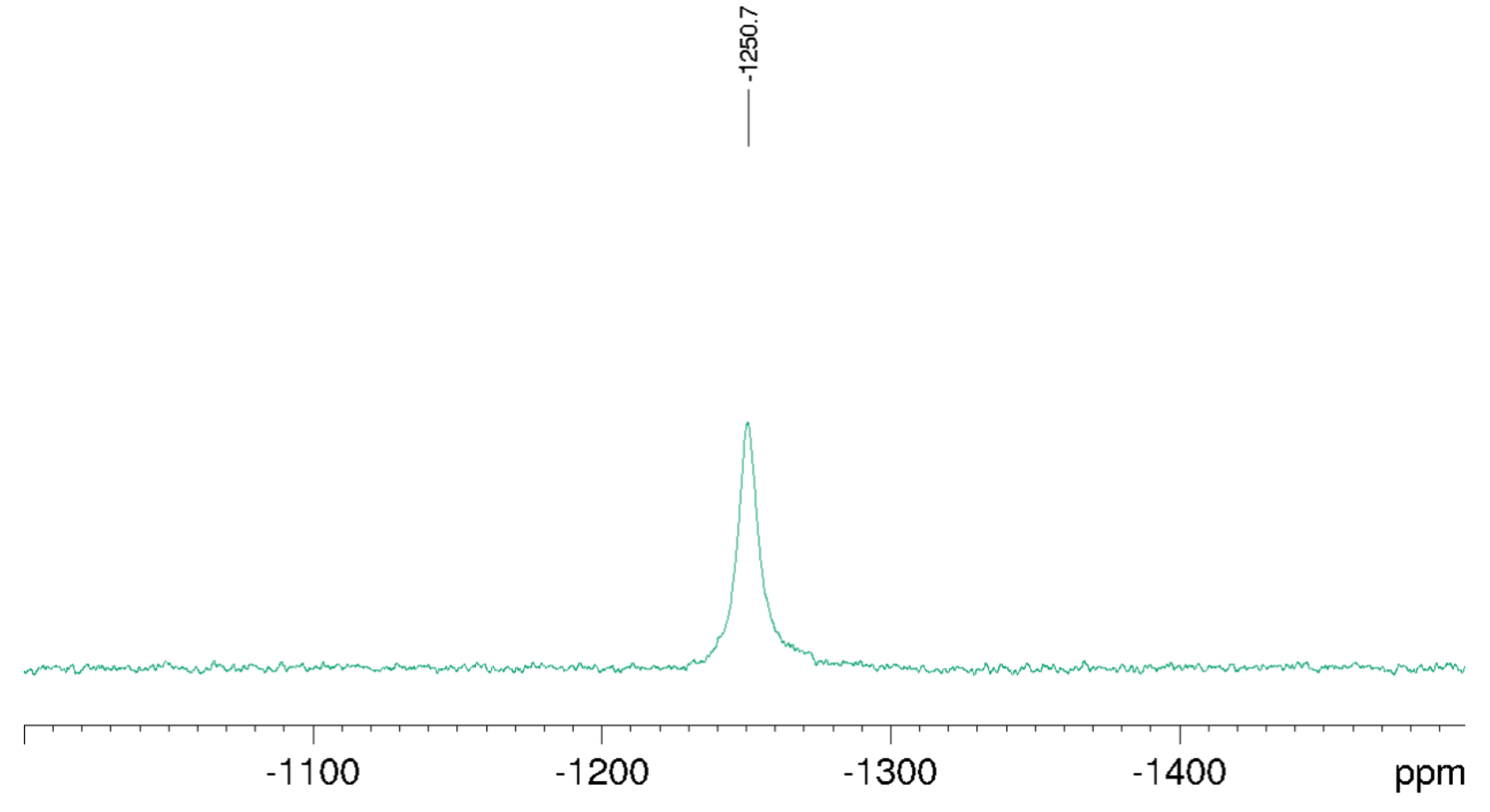

Figure S7. High-field ${ }^{2} \mathrm{H}$ NMR spectrum $\left(61.31 \mathrm{MHz}, \mathrm{C}_{6} \mathrm{H}_{6}, 295 \mathrm{~K}\right)$ of complex 4-D.

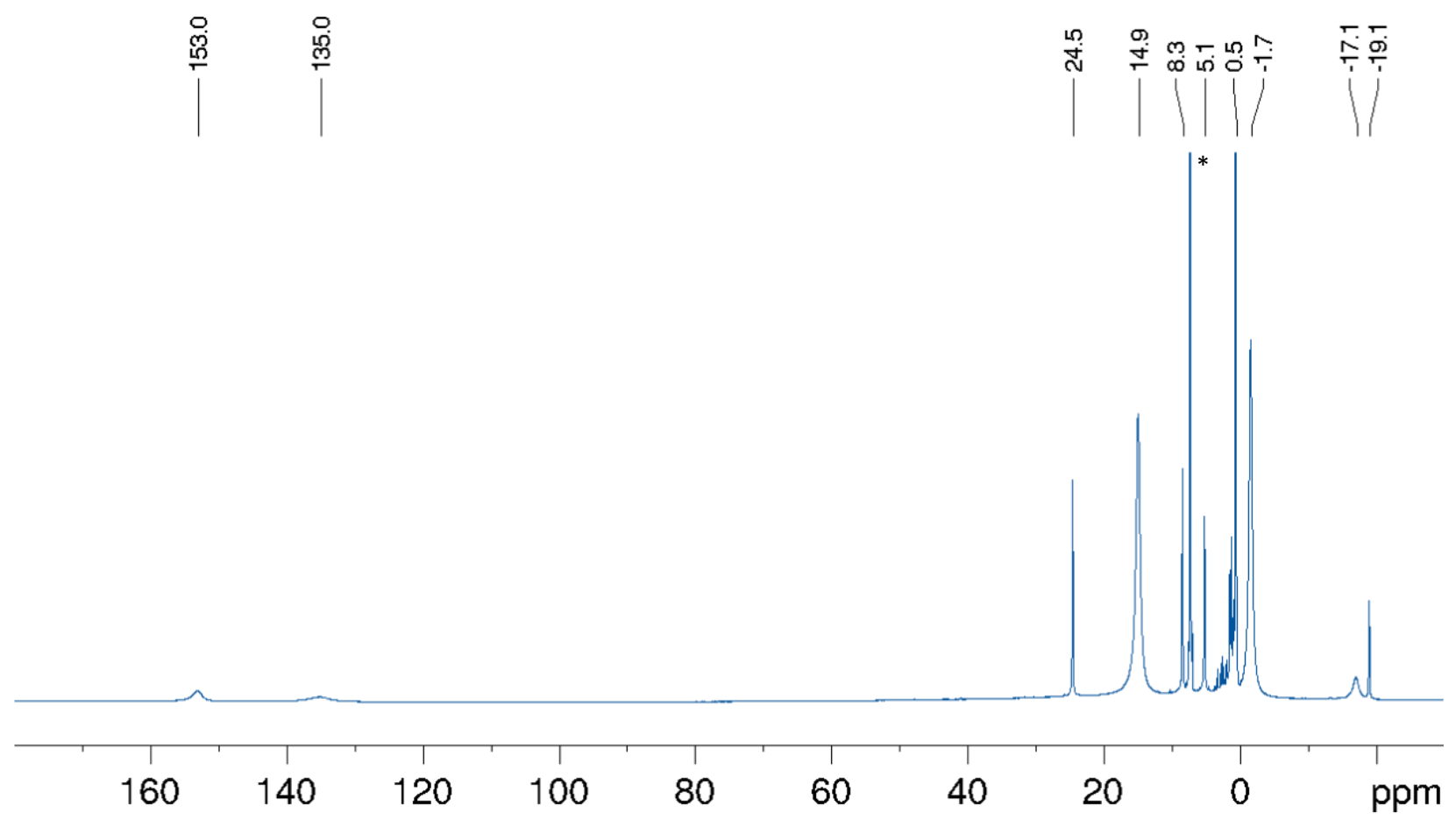

Figure S8. ${ }^{1} \mathrm{H}$ NMR spectrum $\left(399.89 \mathrm{MHz}, \mathrm{C}_{6} \mathrm{D}_{6}, 295 \mathrm{~K}\right)$ of complex 4. The resonance of the solvent is indicated by an asterisk. 


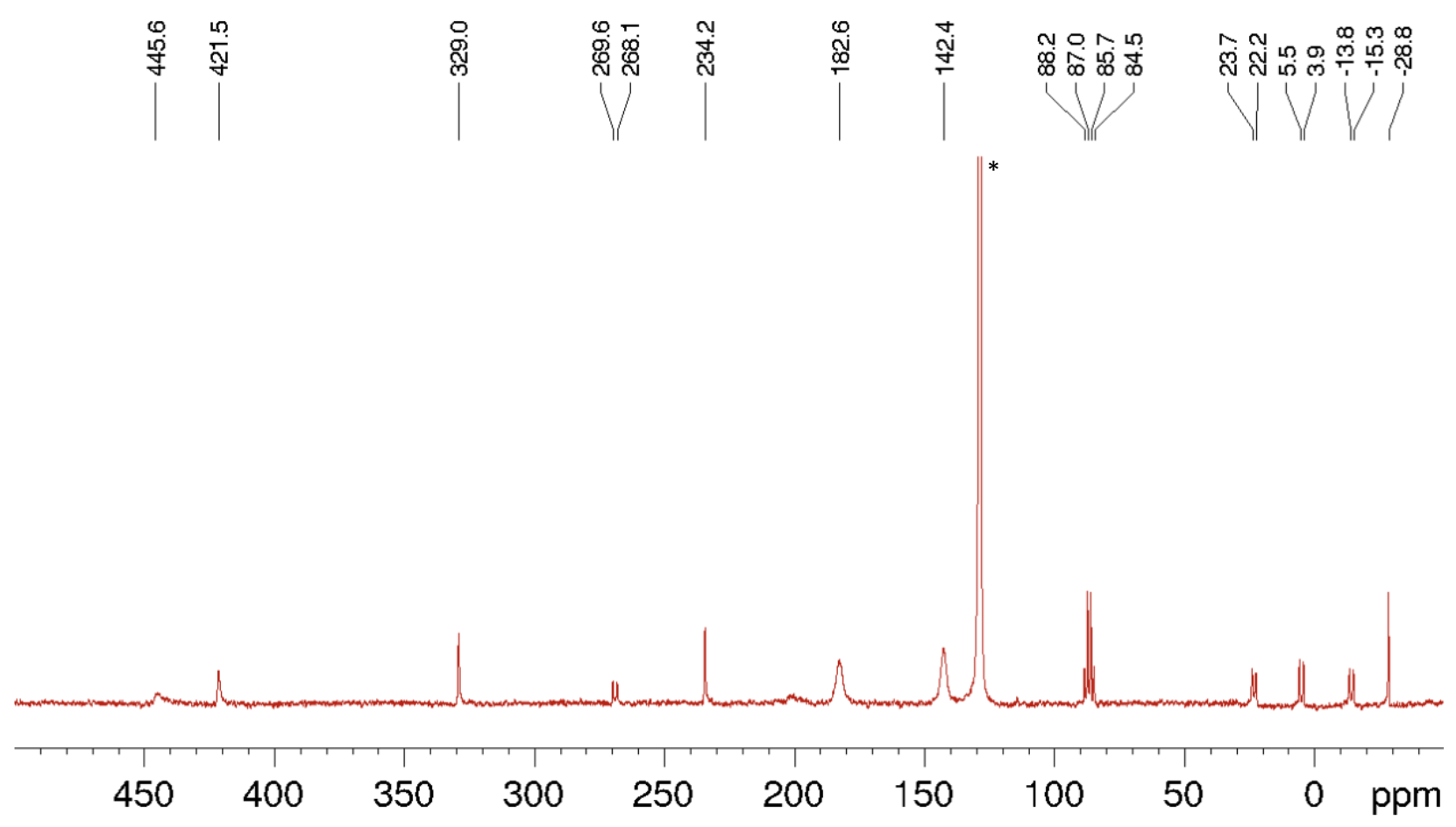

Figure S9. ${ }^{13} \mathrm{C}$ NMR spectrum $\left(100.57 \mathrm{MHz}, \mathrm{C}_{6} \mathrm{D}_{6}, 295 \mathrm{~K}\right)$ of complex 4 . The resonance of the solvent is indicated by an asterisk.

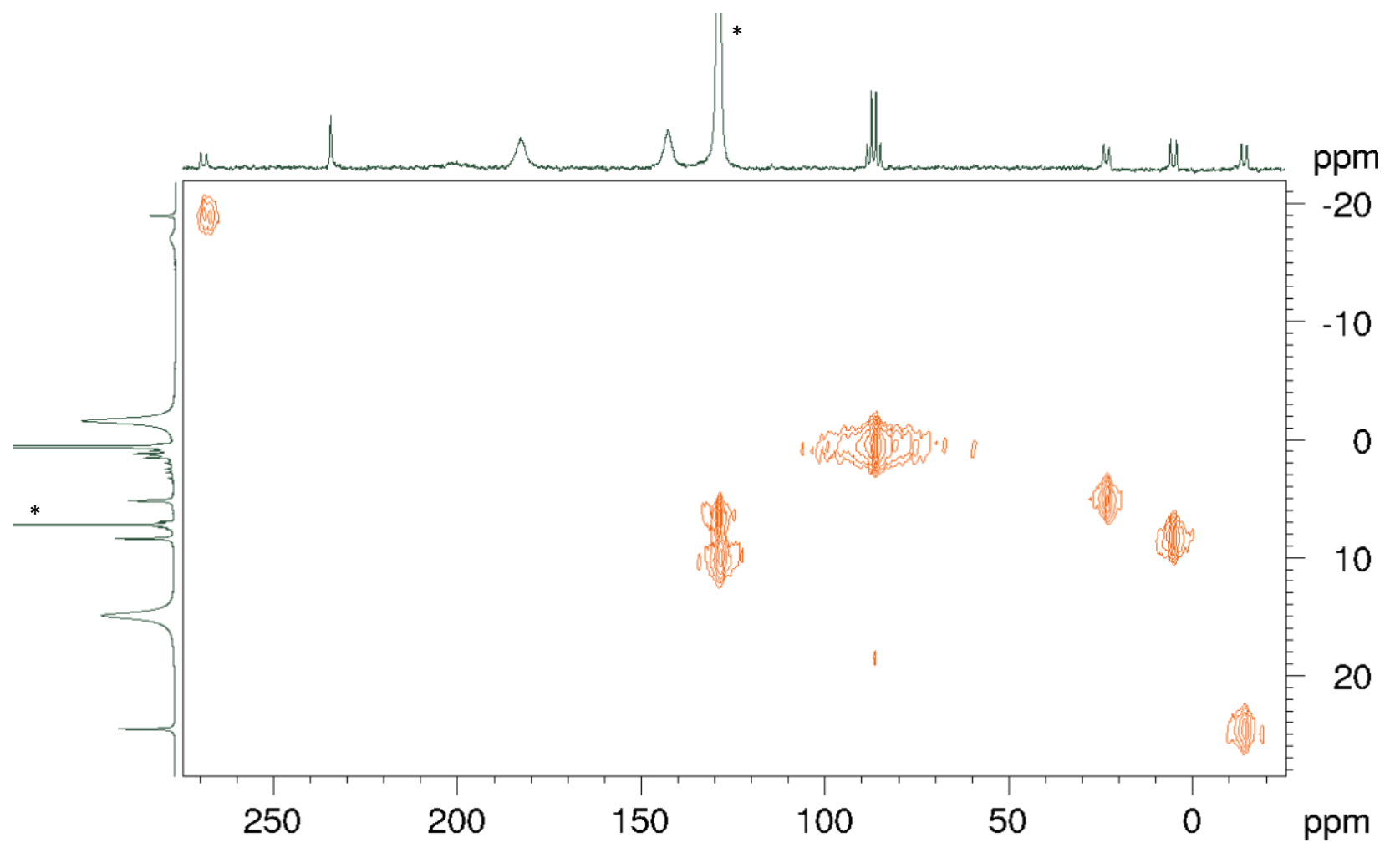

Figure S10. ${ }^{13} \mathrm{C}-{ }^{1} \mathrm{H}$ HETCOR NMR spectrum (100.57 MHz \& 399.89 MHz, C6 $\left.\mathrm{D}_{6}, 295 \mathrm{~K}\right)$ of complex 4. The resonances of the solvent are indicated by asterisks. 


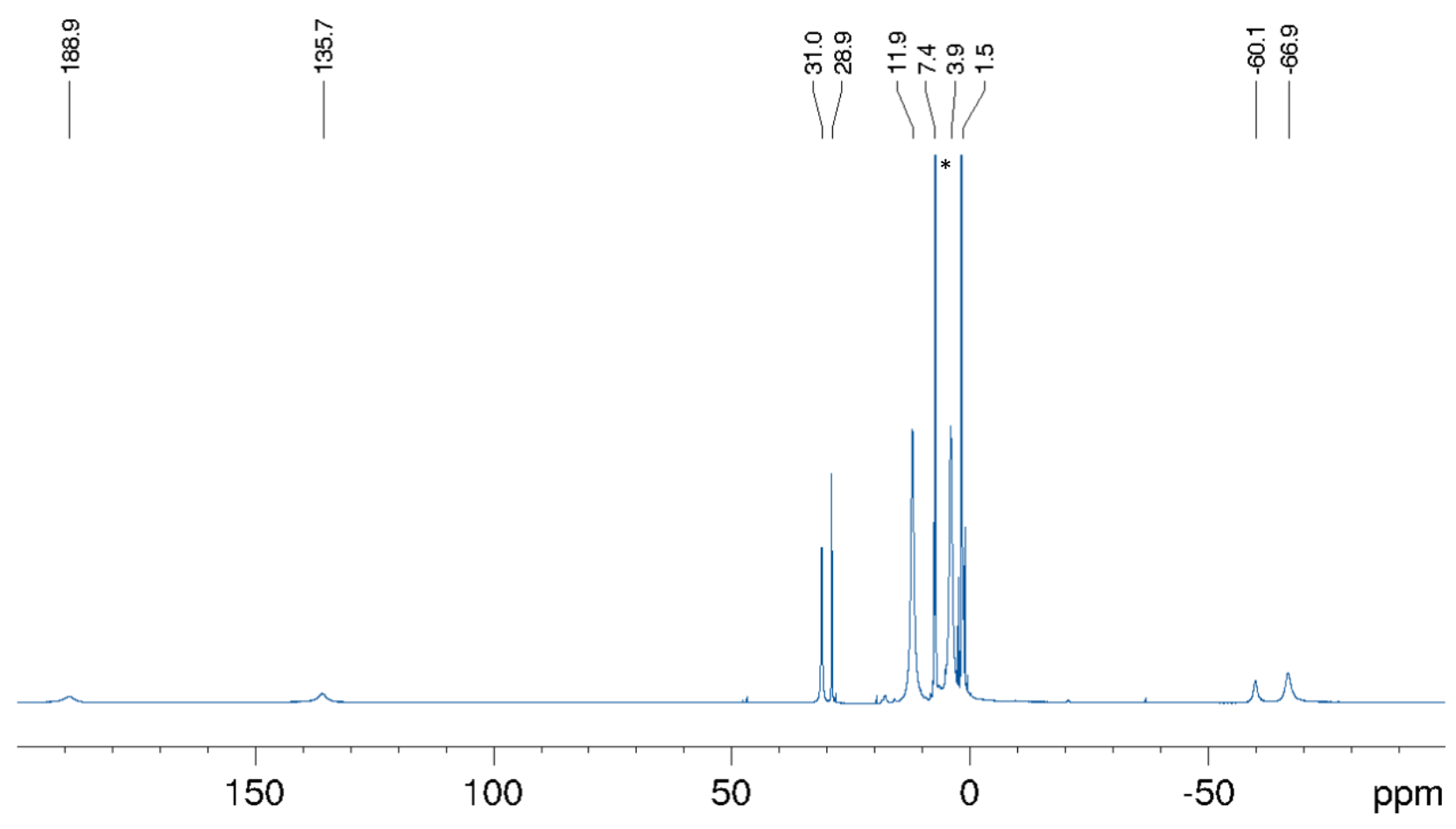

Figure S11. ${ }^{1} \mathrm{H}$ NMR spectrum $\left(600.13 \mathrm{MHz}, \mathrm{C}_{6} \mathrm{D}_{6}, 295 \mathrm{~K}\right)$ of complex 5 . The resonance of the solvent is indicated by an asterisk.

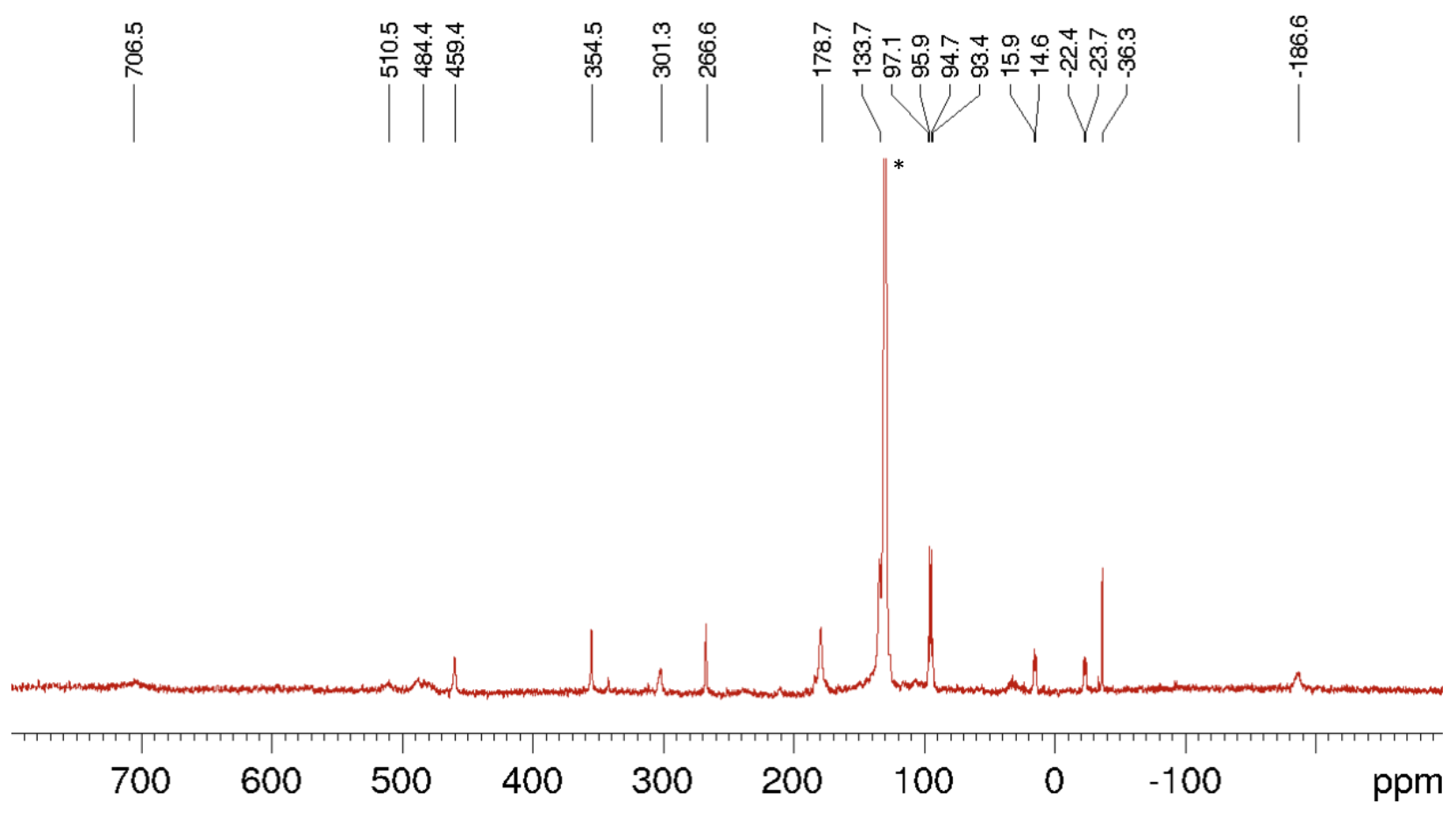

Figure S12. ${ }^{13} \mathrm{C}$ NMR spectrum $\left(100.61 \mathrm{MHz}, \mathrm{C}_{6} \mathrm{D}_{6}, 295 \mathrm{~K}\right)$ of complex 5. The resonance of the solvent is indicated by an asterisk. 


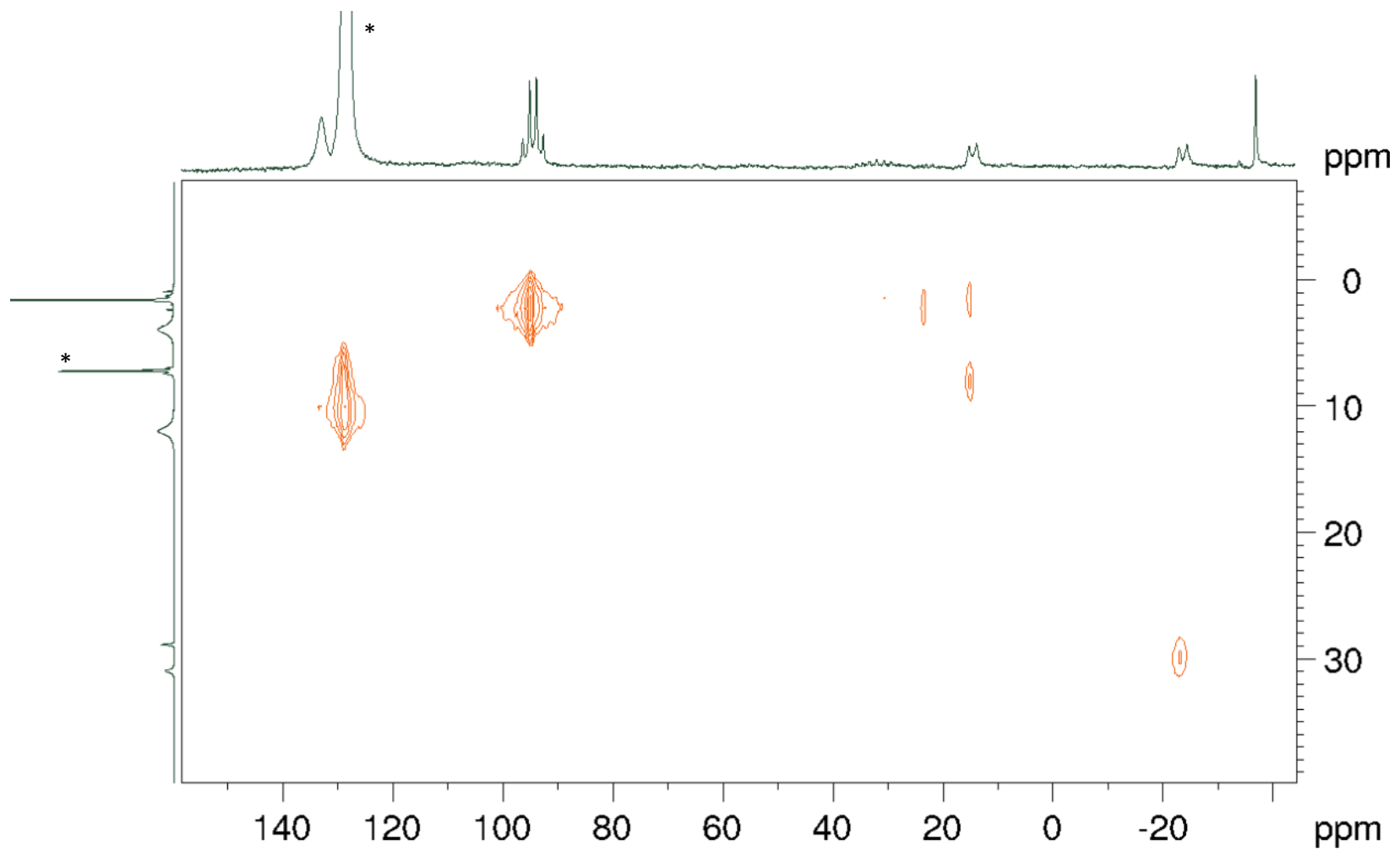

Figure S13. ${ }^{13} \mathrm{C}-{ }^{1} \mathrm{H}$ HETCOR NMR spectrum $\left(100.57 \mathrm{MHz} \& 399.89 \mathrm{MHz}, \mathrm{C}_{6} \mathrm{D}_{6}, 295 \mathrm{~K}\right)$ of complex 5. The resonances of the solvent are indicated by asterisks.

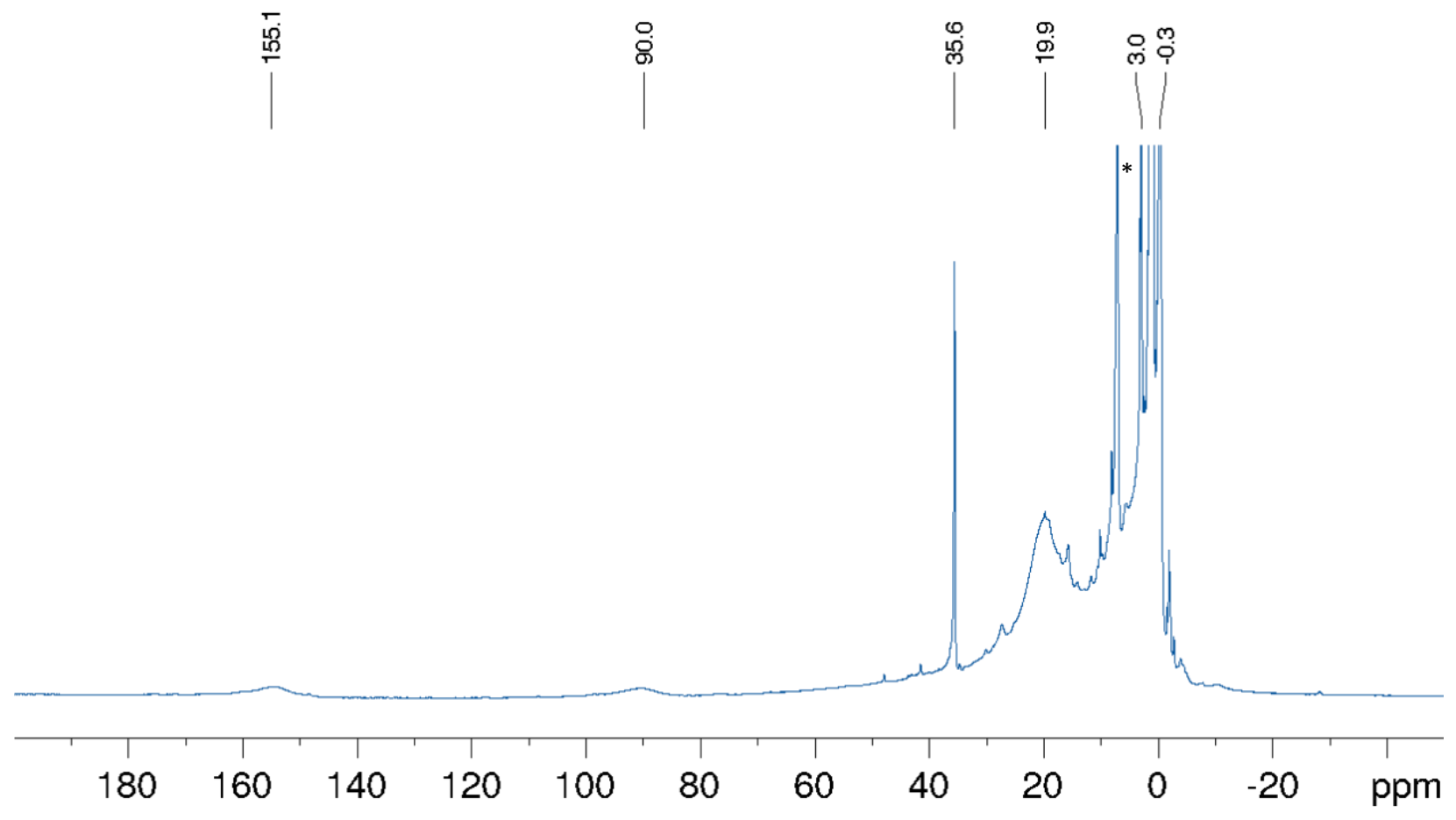

Figure S14. ${ }^{1} \mathrm{H}$ NMR spectrum $\left(399.89 \mathrm{MHz}, \mathrm{C}_{6} \mathrm{D}_{6}, 295 \mathrm{~K}\right)$ of complex 7. The resonance of the solvent is indicated by an asterisk. 


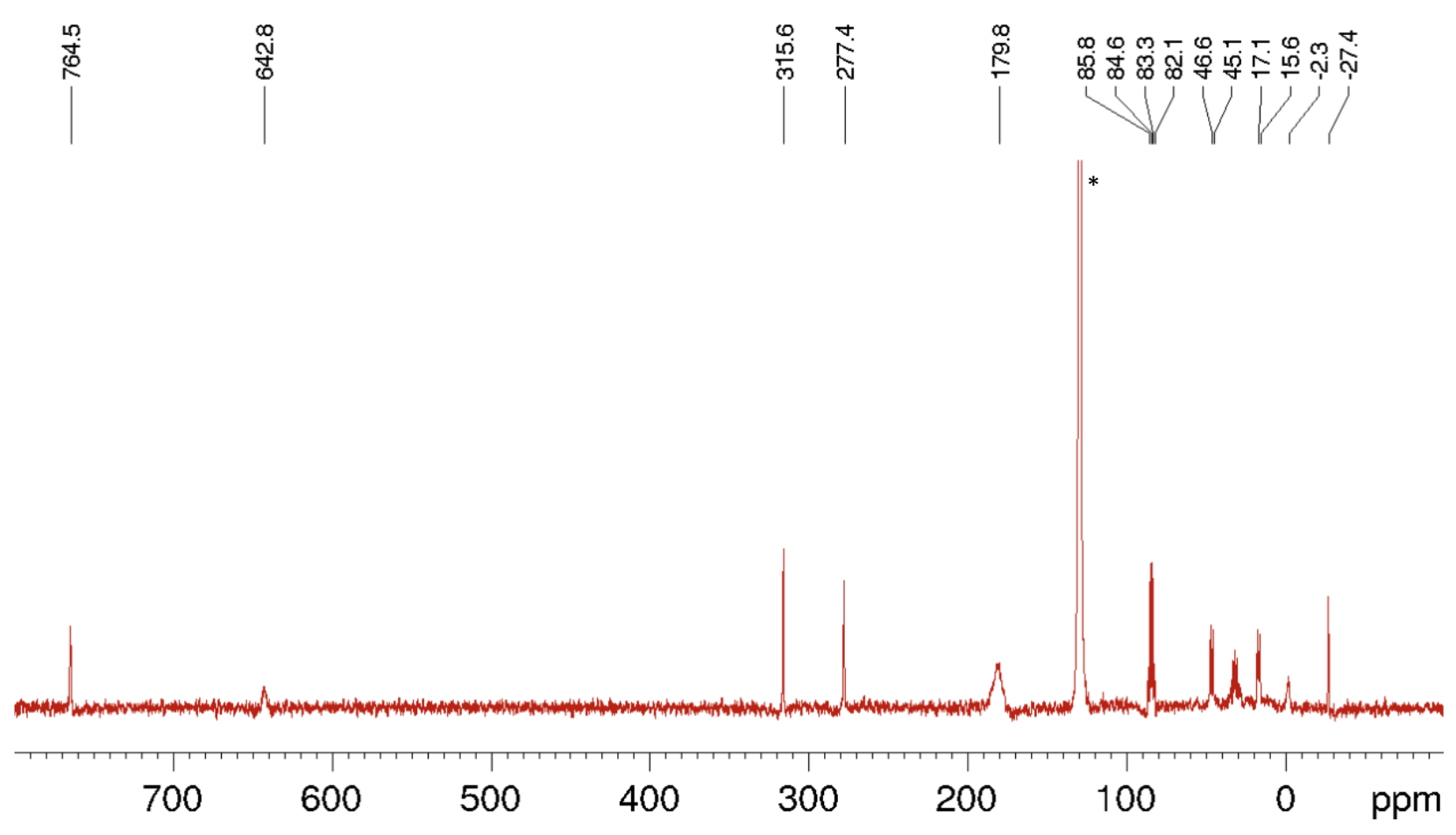

Figure S15. ${ }^{13} \mathrm{C}$ NMR spectrum $\left(100.61 \mathrm{MHz}, \mathrm{C}_{6} \mathrm{D}_{6}, 295 \mathrm{~K}\right)$ of complex 7. The resonance of the solvent is indicated by an asterisk.

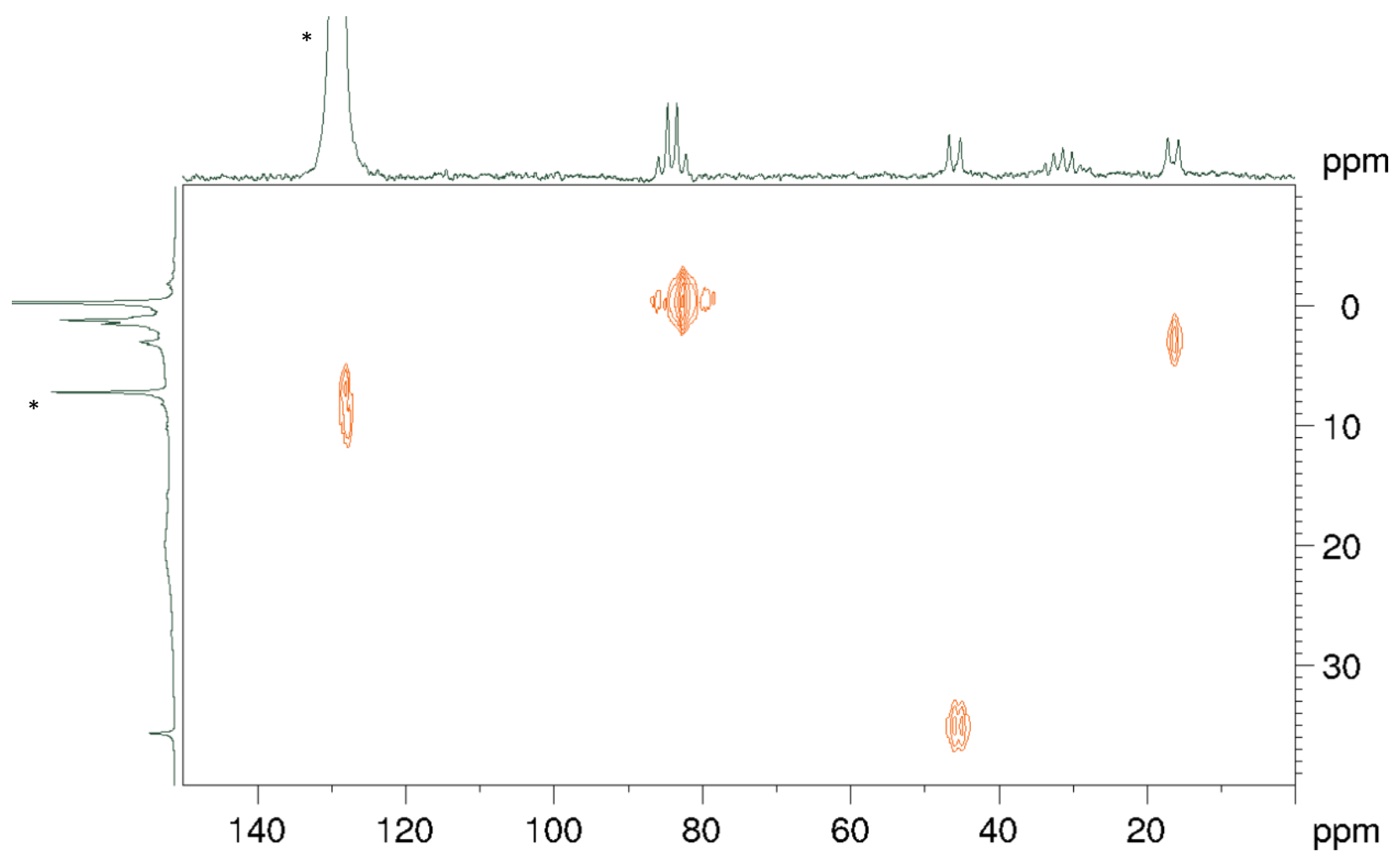

Figure S16. ${ }^{13} \mathrm{C}-{ }^{1} \mathrm{H}$ HETCOR NMR spectrum (100.57 MHz \& $\left.399.89 \mathrm{MHz}, \mathrm{C}_{6} \mathrm{D}_{6}, 295 \mathrm{~K}\right)$ of complex 7. The resonances of the solvent are indicated by asterisks. 


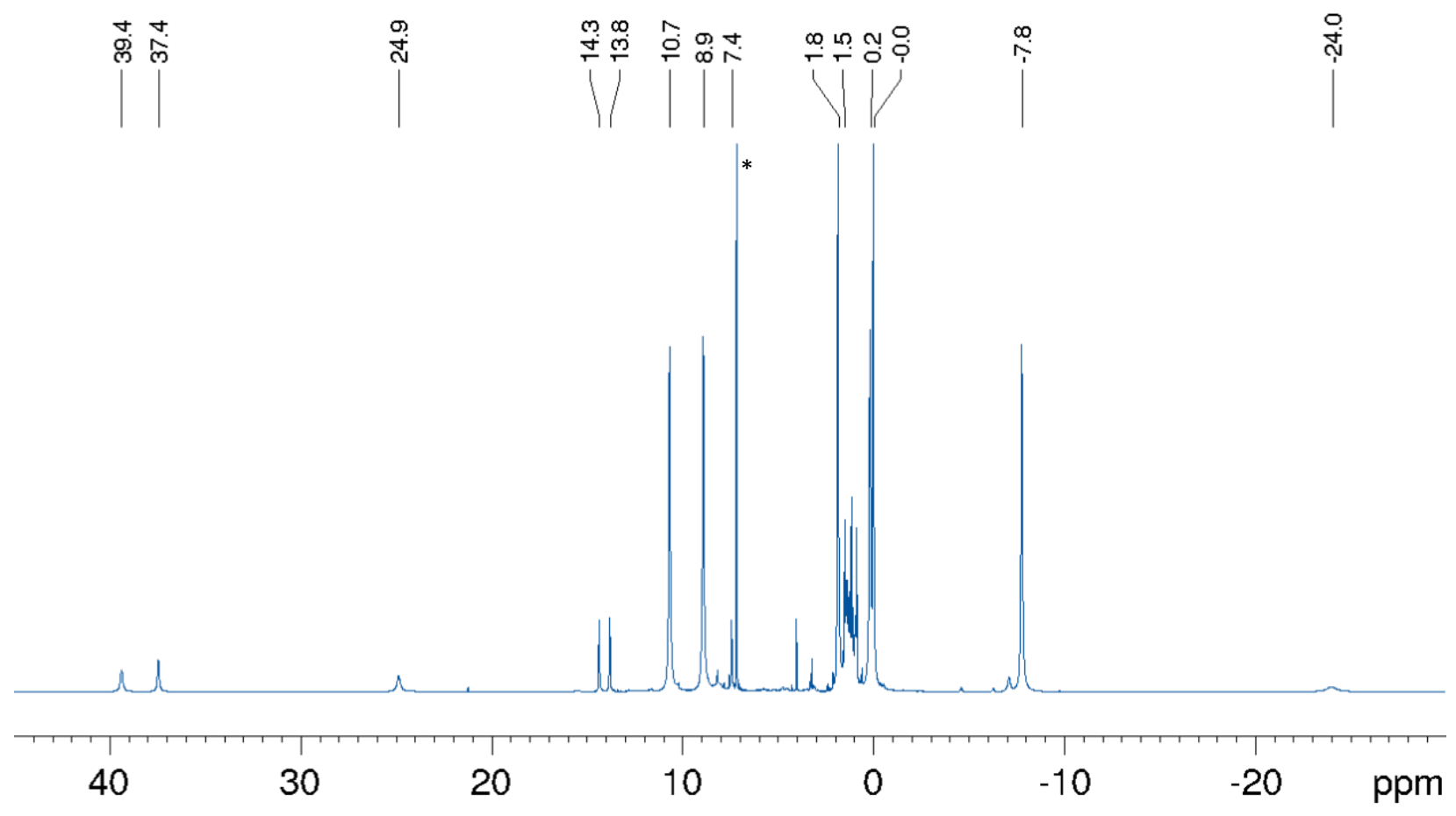

Figure S17. ${ }^{1} \mathrm{H}$ NMR spectrum $\left(600.13 \mathrm{MHz}, \mathrm{C}_{6} \mathrm{D}_{6}, 295 \mathrm{~K}\right)$ of complex 8 . The resonance of the solvent is indicated by an asterisk.

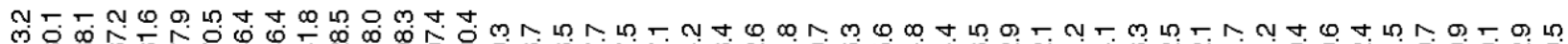

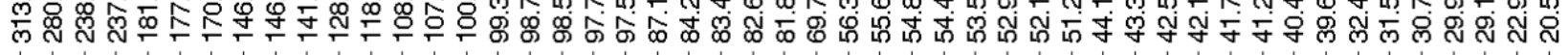

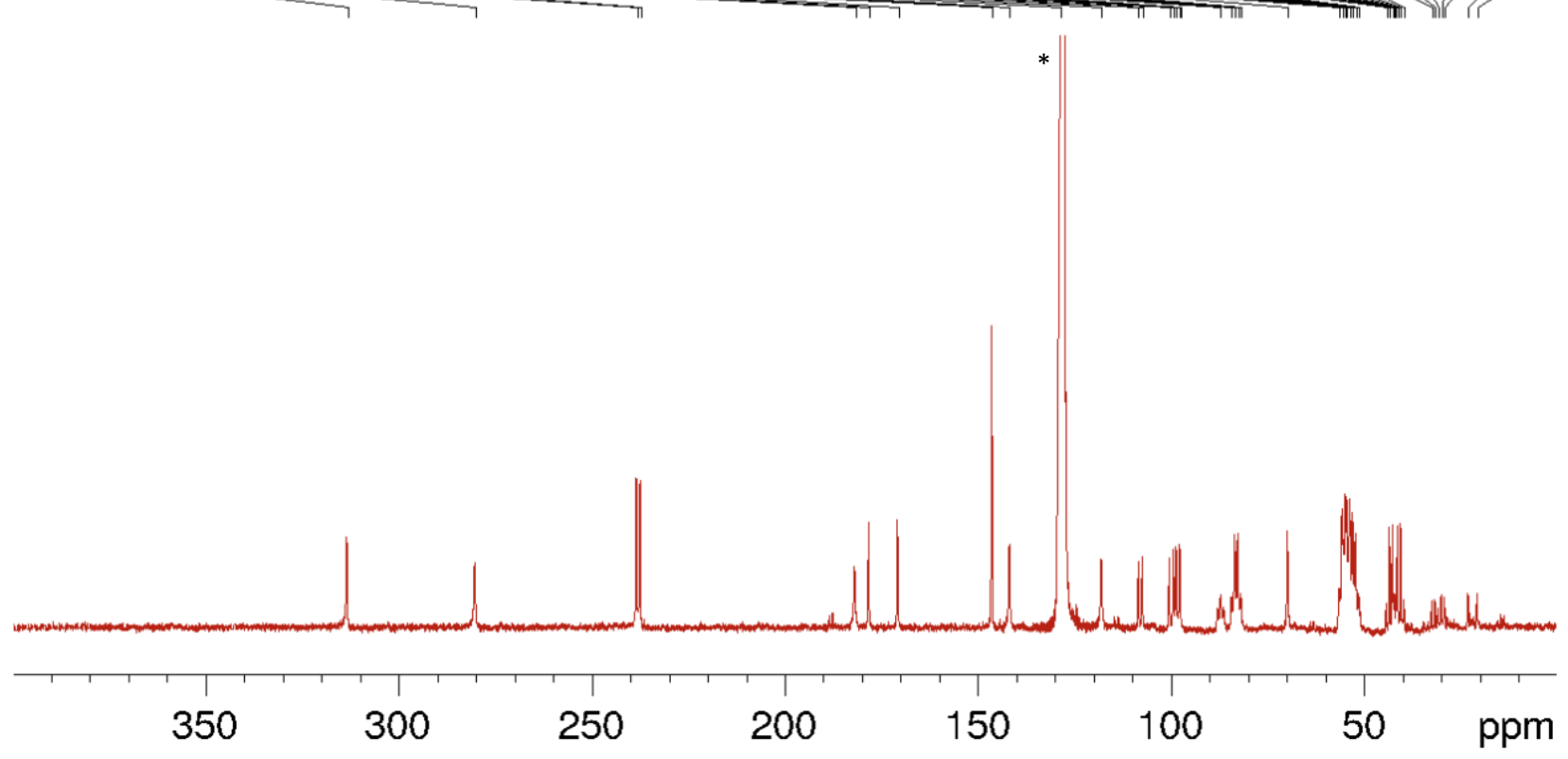

Figure S18. ${ }^{13} \mathrm{C}$ NMR spectrum $\left(150.92 \mathrm{MHz}, \mathrm{C}_{6} \mathrm{D}_{6}, 295 \mathrm{~K}\right)$ of complex 8 . The resonance of the solvent is indicated by an asterisk. 


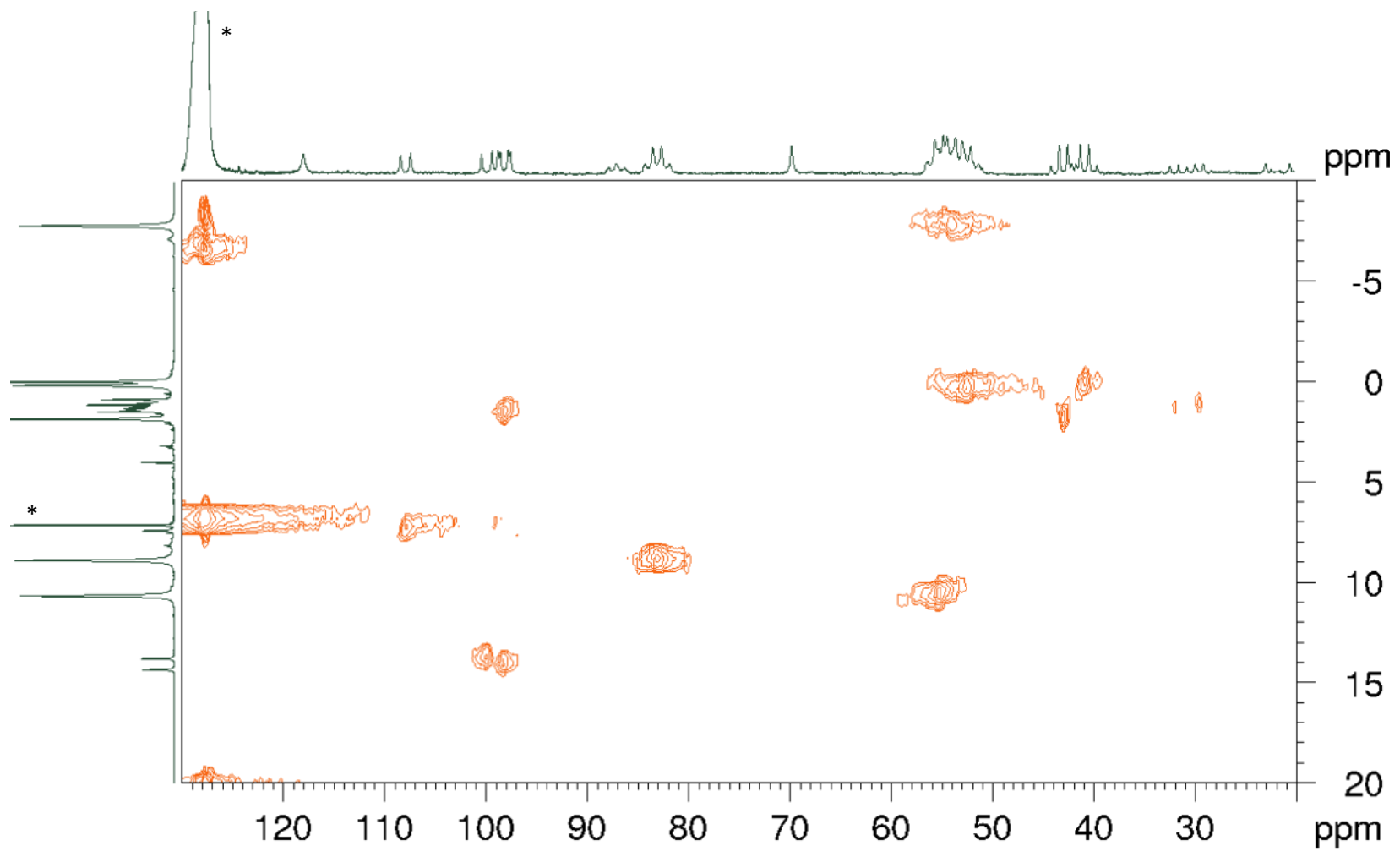

Figure S19. ${ }^{13} \mathrm{C}-{ }^{1} \mathrm{H}$ HETCOR NMR spectrum $\left(150.92 \mathrm{MHz} \& 600.13 \mathrm{MHz}, \mathrm{C}_{6} \mathrm{D}_{6}, 295 \mathrm{~K}\right)$ of complex 8. The resonances of the solvent are indicated by asterisk.

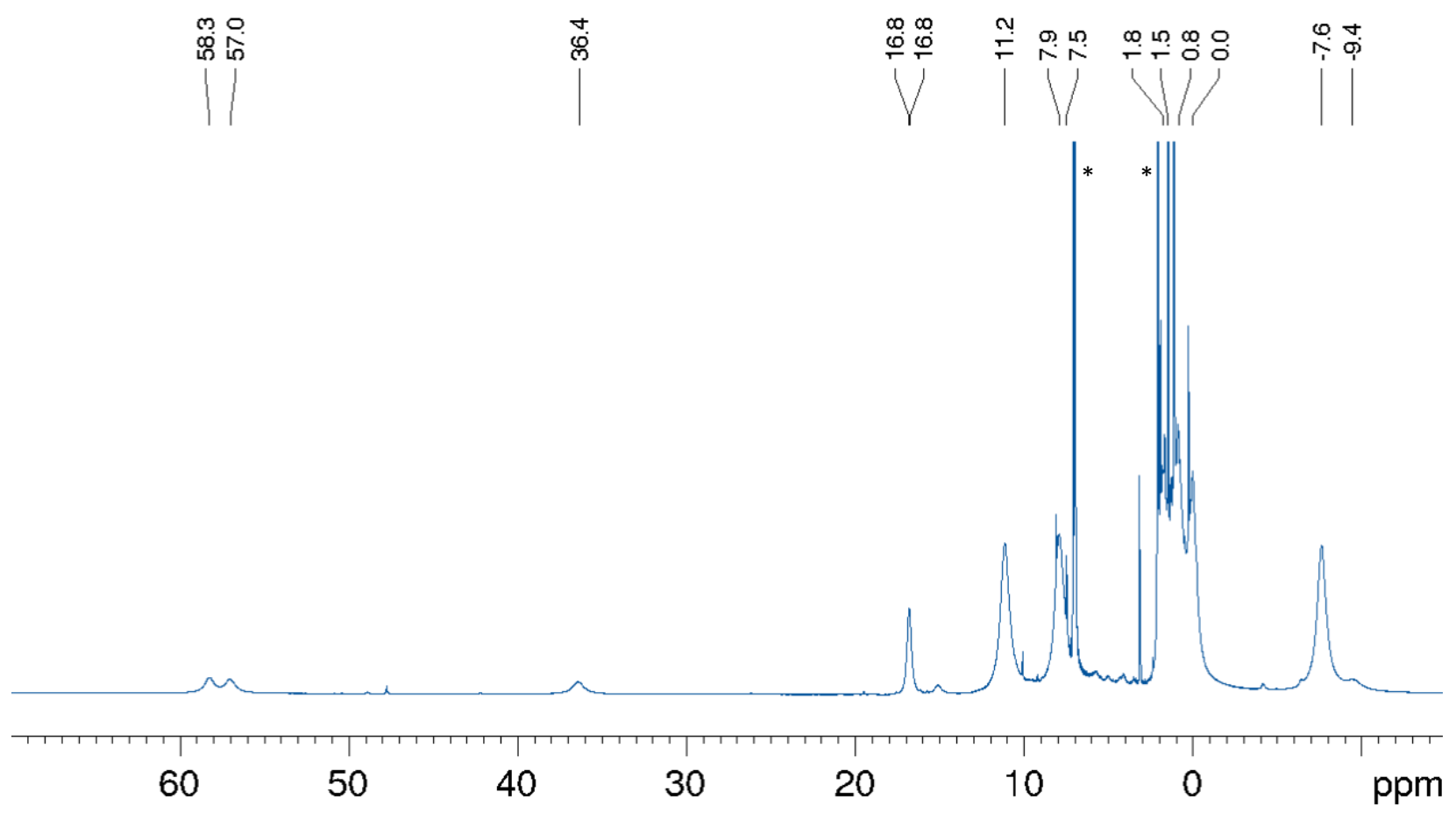

Figure S20. ${ }^{1} \mathrm{H}$ NMR spectrum $\left(600.13 \mathrm{MHz}, \mathrm{Tol}-d^{8}, 295 \mathrm{~K}\right)$ of complex 9. The resonances of the solvent are indicated by asterisks. 


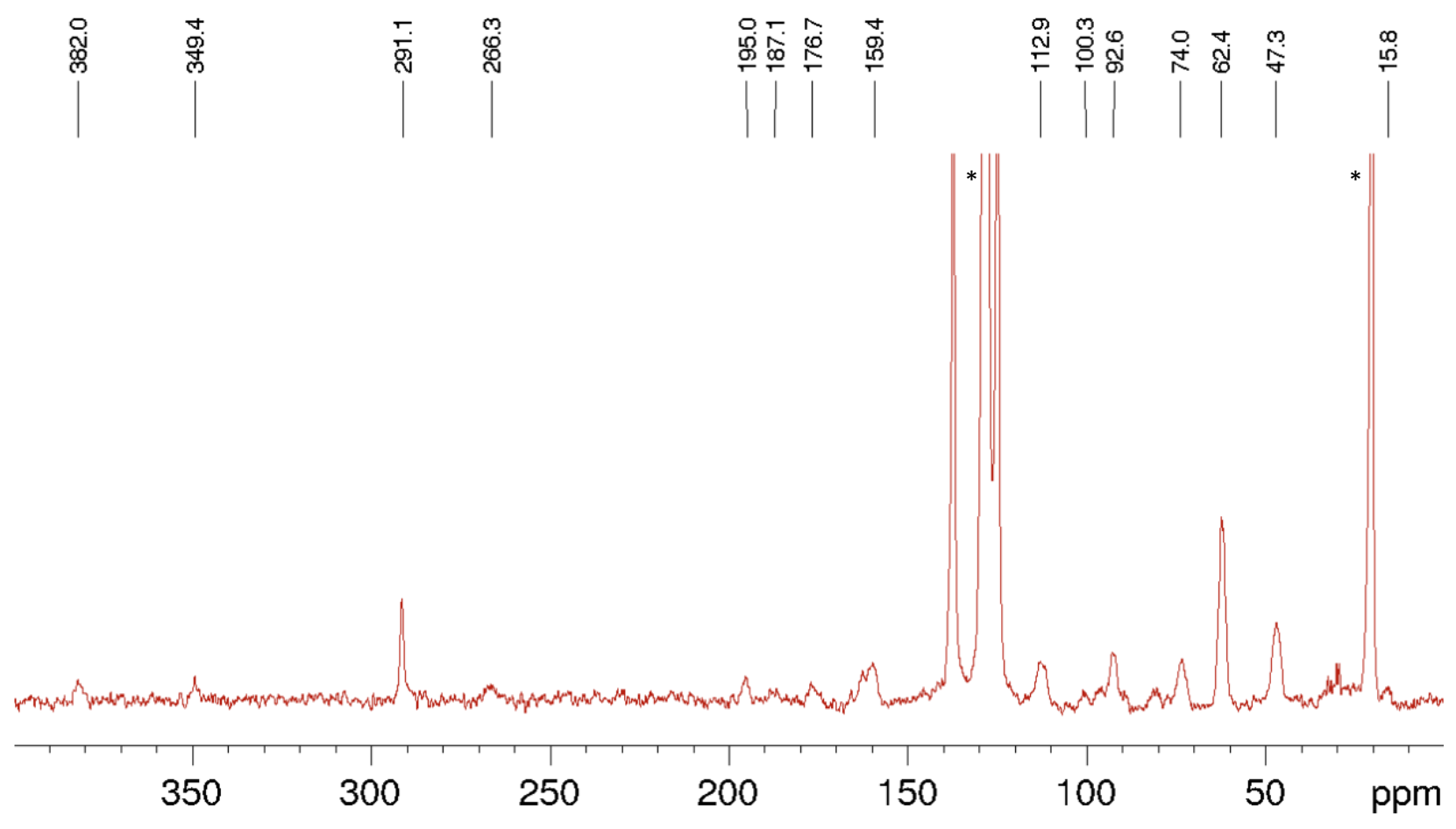

Figure S21. ${ }^{13} \mathrm{C}$ NMR spectrum $\left(150.92 \mathrm{MHz}, \mathrm{Tol}-d^{8}, 295 \mathrm{~K}\right)$ of complex 9. The resonances of the solvent are indicated by asterisks. 


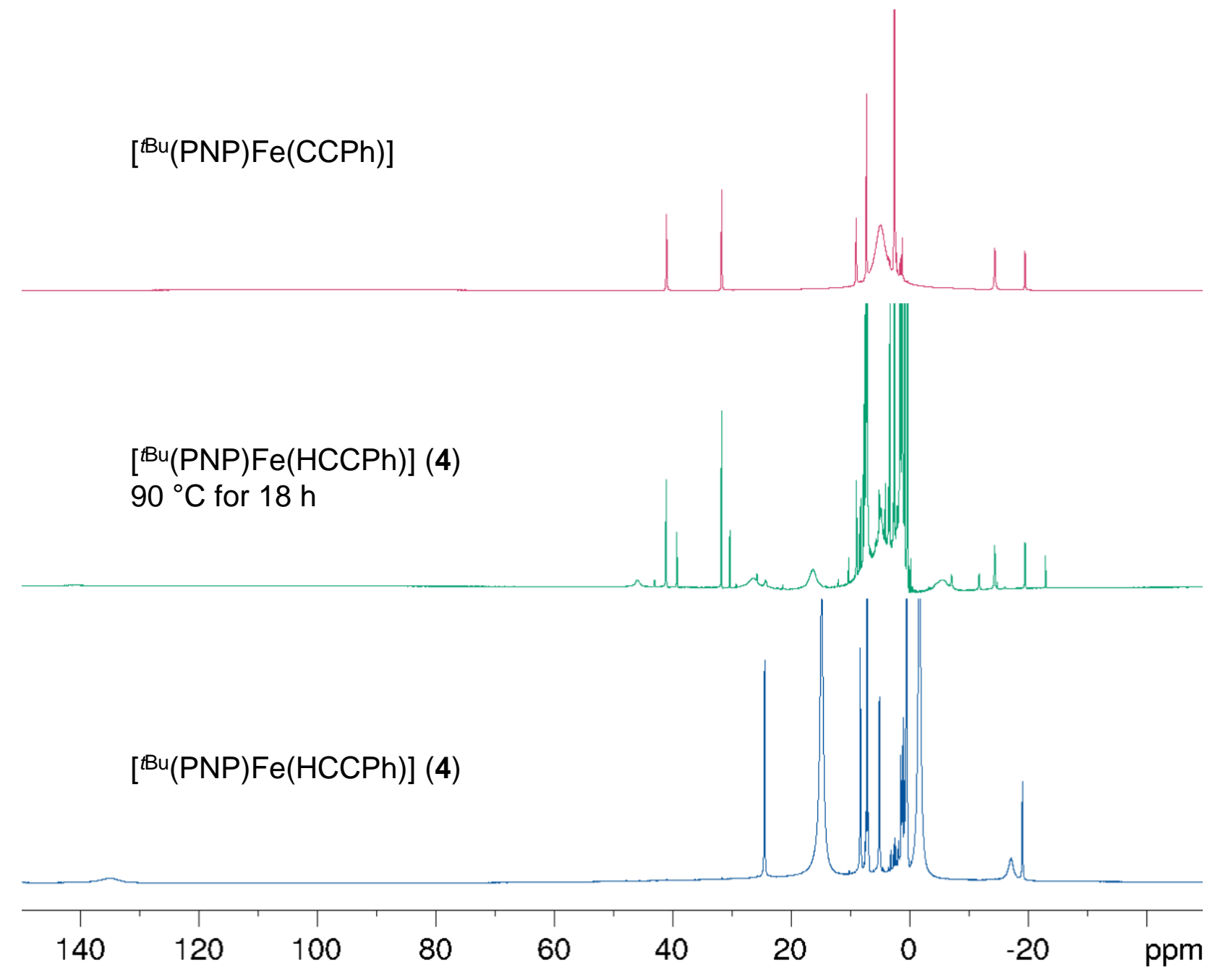

Figure S22. Comparison of the ${ }^{1} \mathrm{H}$ NMR spectra $\left(600.13 \mathrm{MHz}, \mathrm{C}_{6} \mathrm{D}_{6}, 295 \mathrm{~K}\right)$ of complex 4 (blue), the thermolysis product of complex 4 (green) and ${ }^{t \mathrm{Bu}}(\mathrm{PNP}) \mathrm{Fe}(\mathrm{CCPh})$ (red). 


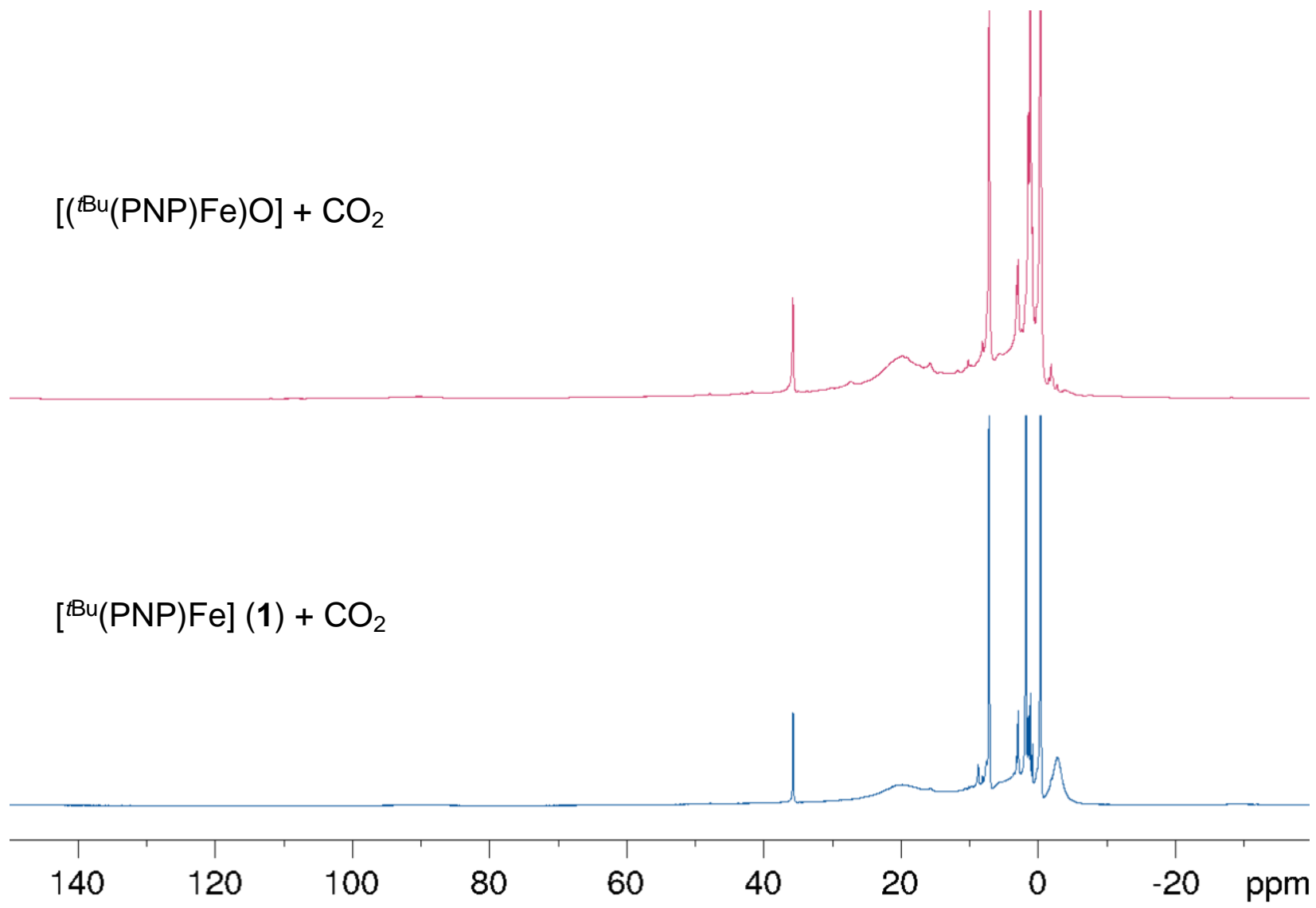

Figure S23. Comparison of the ${ }^{1} \mathrm{H}$ NMR spectra $\left(600.13 \mathrm{MHz}, \mathrm{C}_{6} \mathrm{D}_{6}, 295 \mathrm{~K}\right)$ of the reaction of complex 1 with carbon dioxide (blue, lower spectrum) and the reaction of complex $\left.\left[{ }^{t \mathrm{Bu}}(\mathrm{PNP}) \mathrm{Fe}\right)_{2} \mathrm{O}\right]$ with carbon dioxide (red, upper spectrum). 


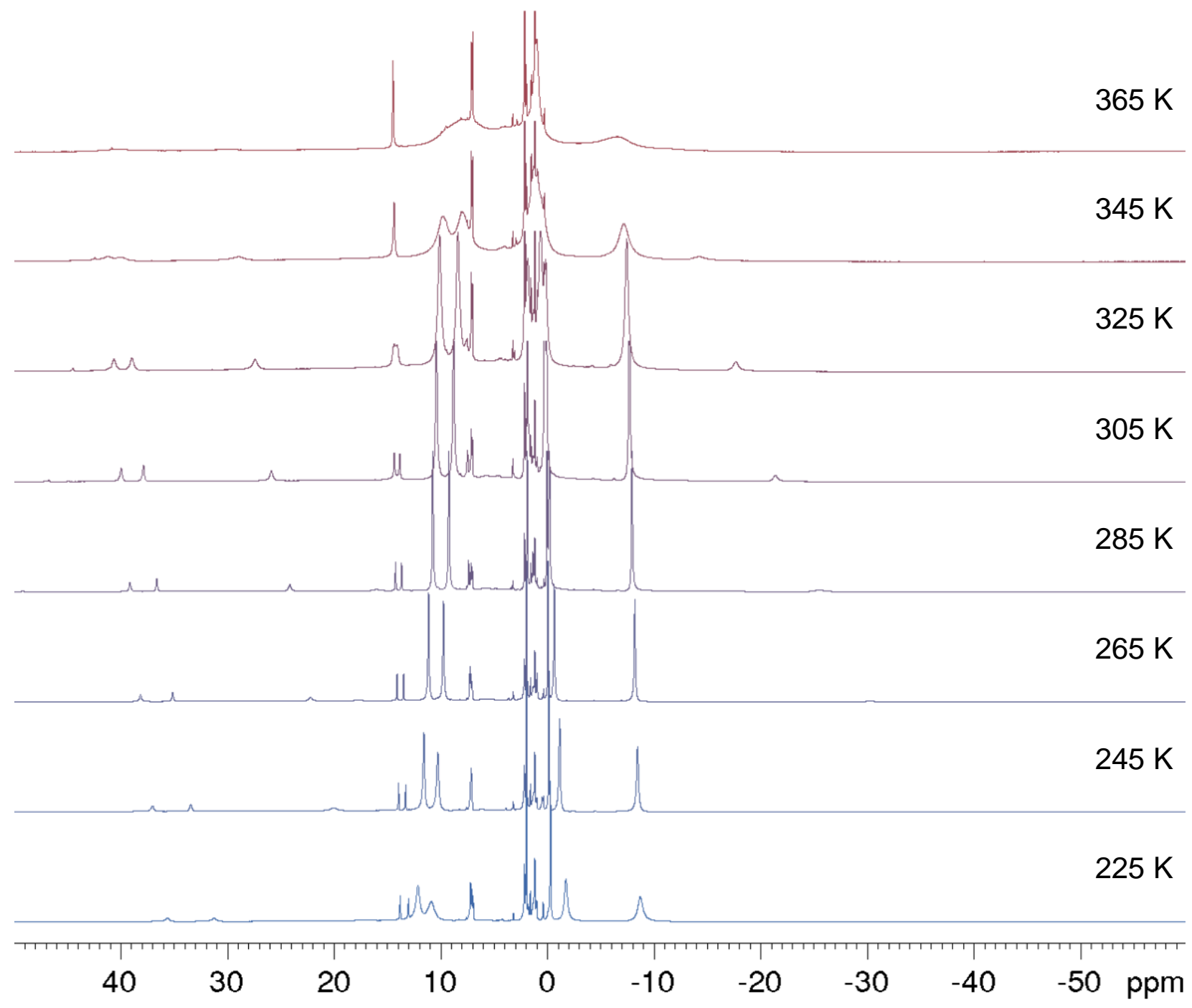

Figure S24. ${ }^{1} \mathrm{H}$ NMR spectra $\left(399.89 \mathrm{MHz}\right.$, Tol- $\left.d^{8}\right)$ of complex 8 at various temperatures. 


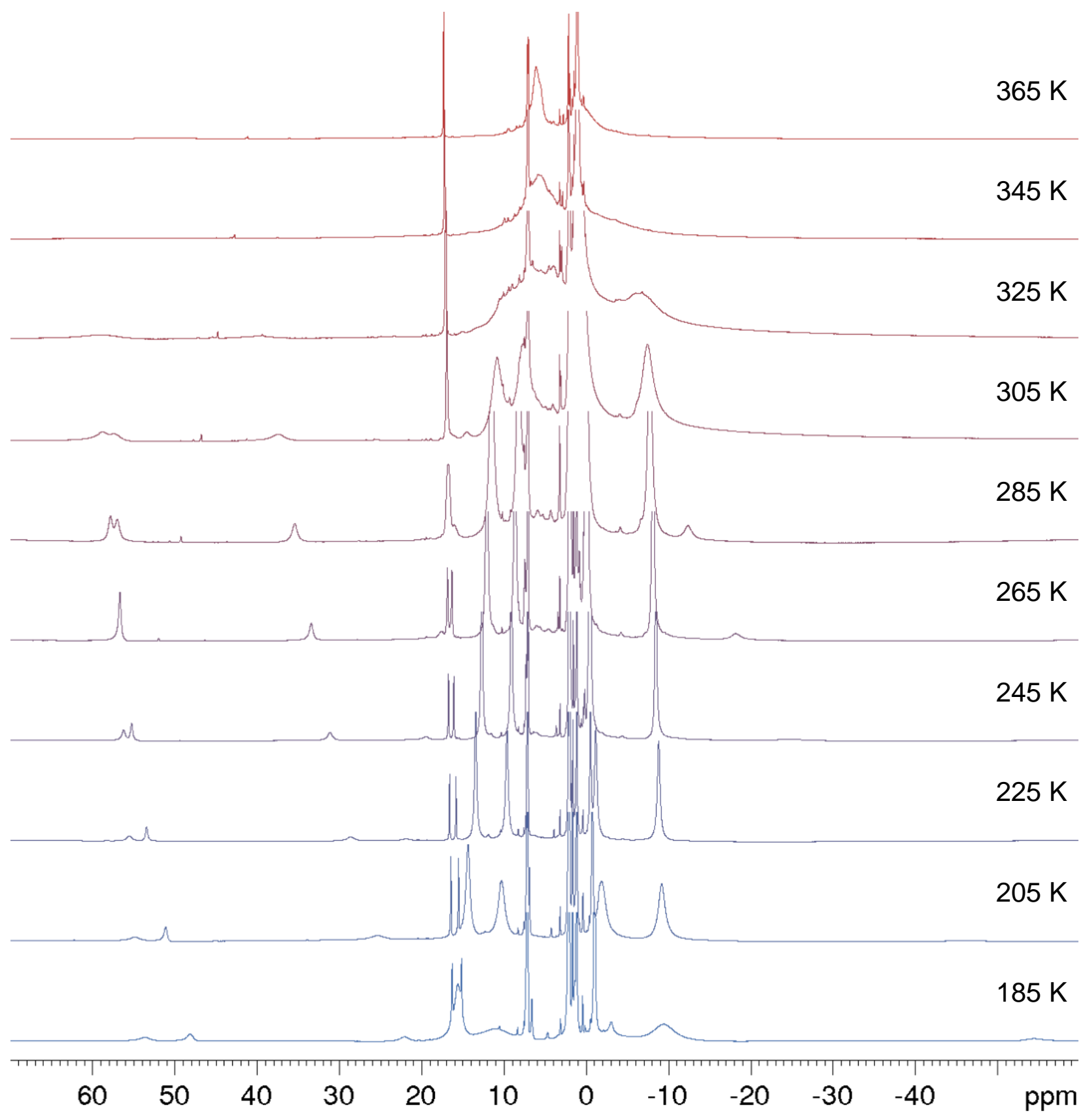

Figure S25. ${ }^{1} \mathrm{H}$ NMR spectra (399.89 MHz, Tol- $\left.d^{8}\right)$ of complex 9 at various temperatures. 


\section{S3 SQUID MAGNETOMETRY}

\section{S3.1 General Procedure}

SQUID measurements were carried out in polycarbonate capsules (Quantum Design, QDS-AGC3). The diamagnetic contribution of the sample was calculated with the aid of Pascal constants and together with the background measured for the empty capsule subtracted from the data. A Quantum Design MPMS XL SQUID magnetometer was used for the measurements. The solid material was powdered (agate motar) before being transferred to the capsule. The filled capsules were then rapidly transferred from an argon filled Schlenk flask to the SQUID magnetometer and evacuated immediately. However, due to the high sensitivity of the measured compounds, the paramagnetic impurities observed in the measurements (Figure S26 and S27) were assigned to a reaction product of the compounds with atmospheric oxygen formed during the transfer into the magnetometer. The fit of the data was performed with the software package $\mathrm{PHI}^{5}$ assuming two coupled $S=2$ centers.

\section{S3.2 MAGNETOMETRIC DATA}

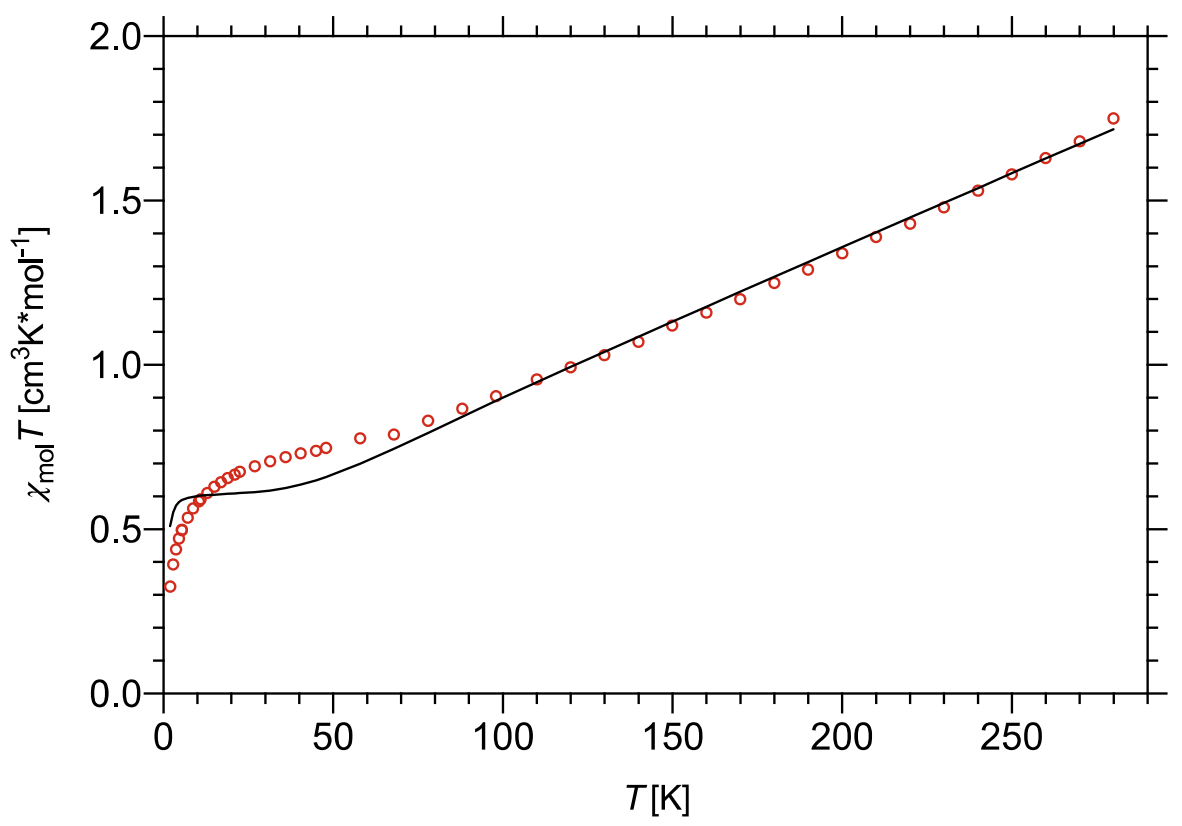

Figure S26. Temperature-dependent SQUID magnetometry of complex 8 recorded at an external field of 1.0 T. The data was fitted using the software package $\mathrm{PHI}^{5}$ (black line) assuming two coupled $S=2$ centers with the inclusion of $20 \%$ of an $S=2$ impurity with the following parameters: $g=2.00 ; J=-68 \mathrm{~cm}^{-1}$, TIP $=$ $5.00 \times 10^{-4} \mathrm{~cm}^{3} / \mathrm{mol}$. 


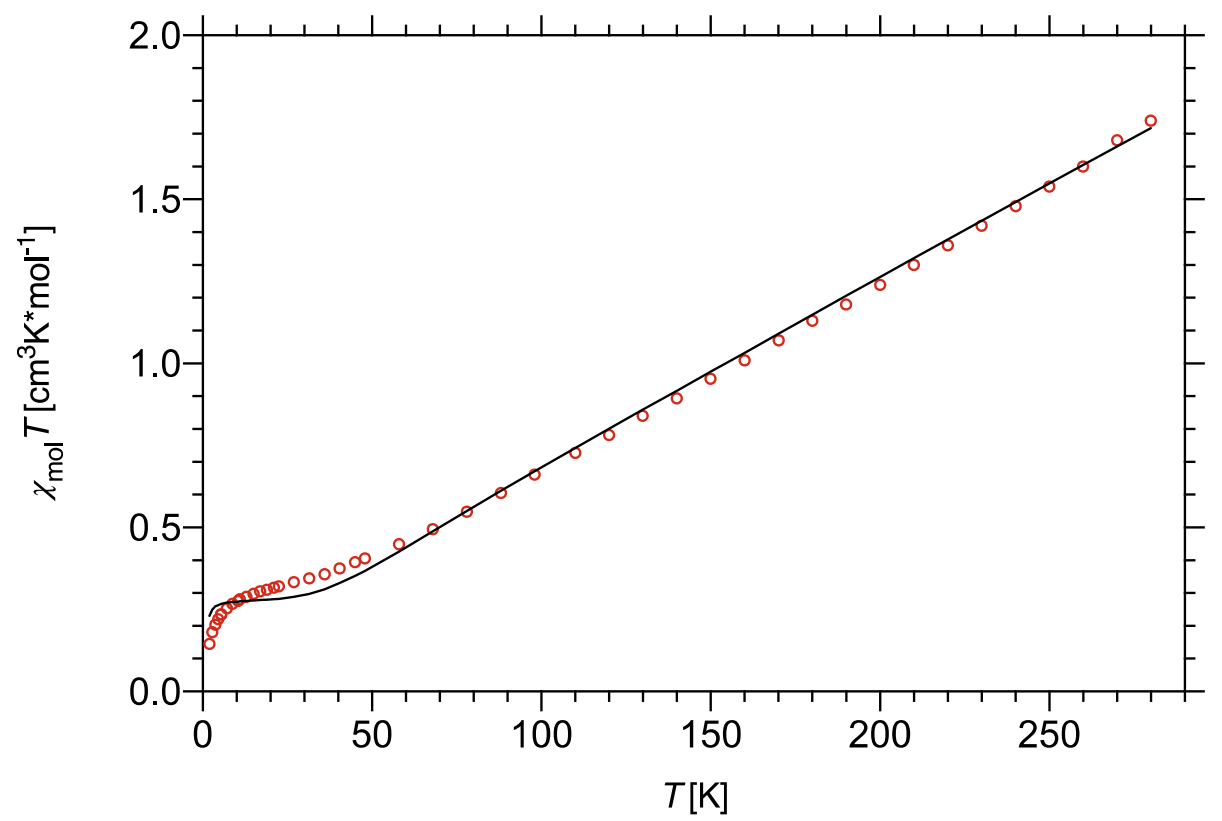

Figure S27. Temperature-dependent SQUID magnetometry of complex 9 recorded at an external field of 1.0 T. The data was fitted using the software package $\mathrm{PHI}^{5}$ (black line) assuming two coupled $S=2$ centers with the inclusion of $9 \%$ of an $S=2$ impurity with the following parameters: $g=1.97 ; J=-58 \mathrm{~cm}^{-1}$, TIP $=$ $5.00 \times 10^{-4} \mathrm{~cm}^{3} / \mathrm{mol}$. 


\section{S4 IR SPECTROSCOPY}

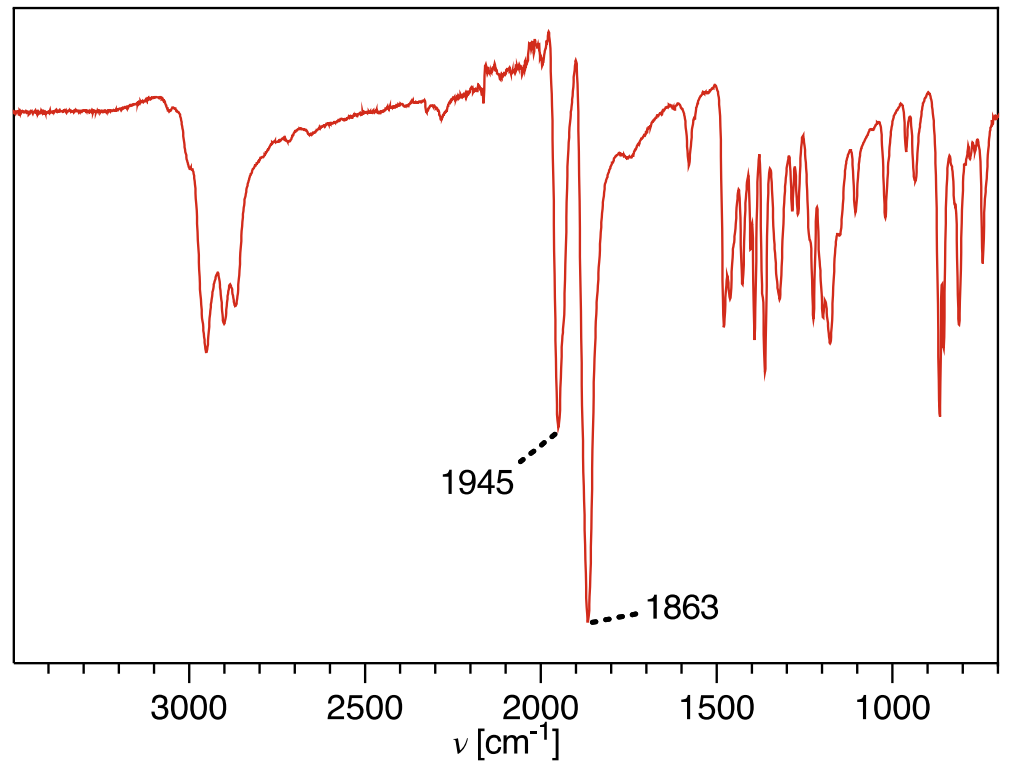

Figure S28. Powder ATR-IR spectrum of complex 3. Numbers indicate peak positions in $\mathrm{cm}^{-1}$.

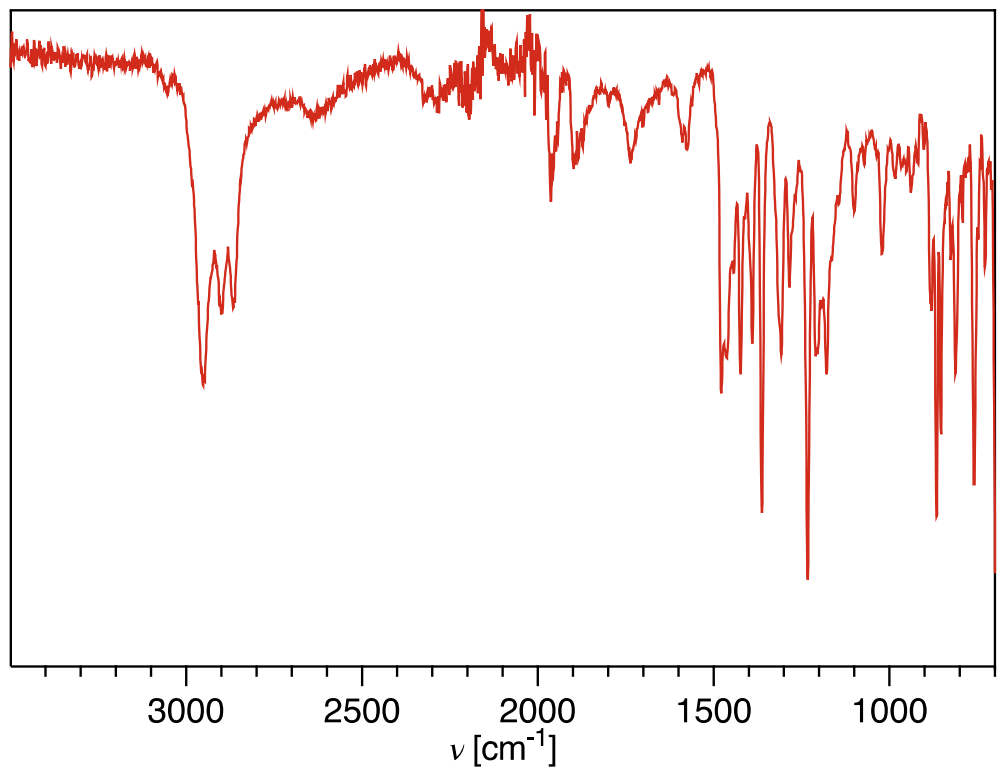

Figure S29. Powder ATR-IR spectrum of complex 4. 


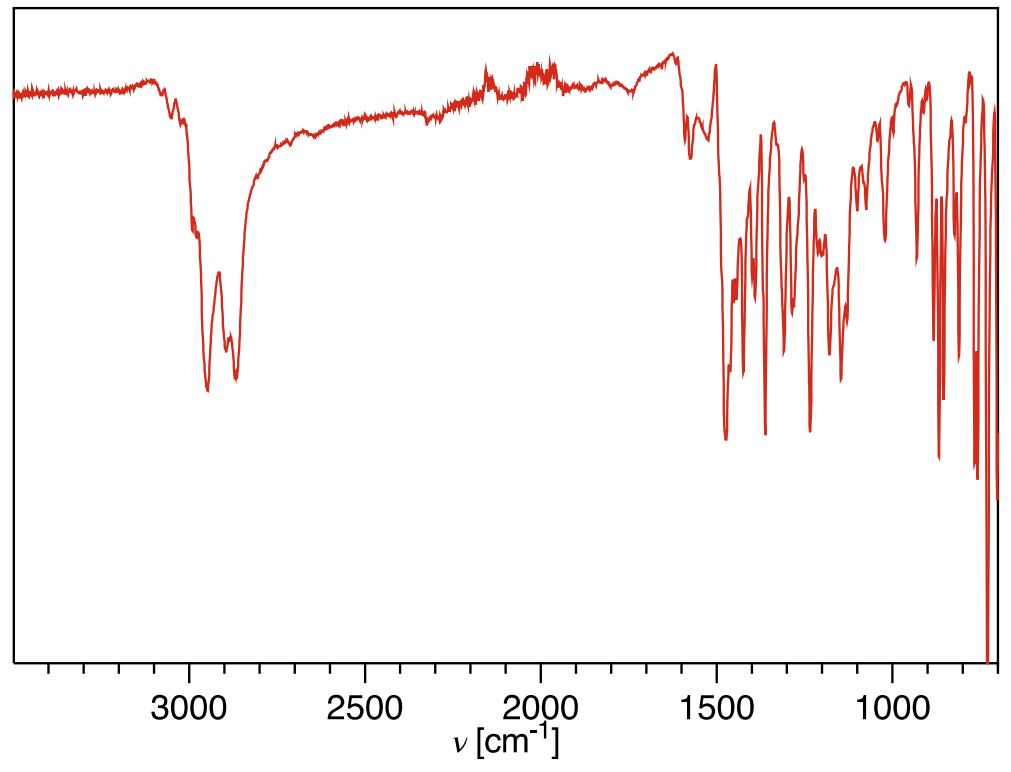

Figure S30. Powder ATR-IR spectrum of complex 5.

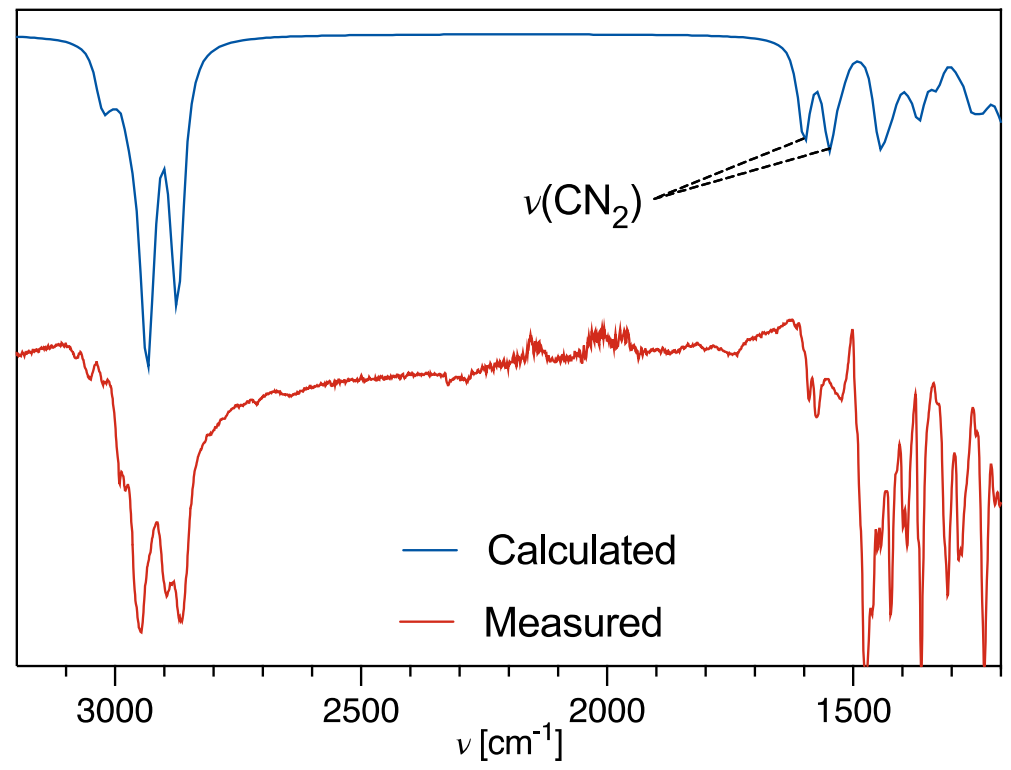

Figure S31. Comparison between calculated (blue, B3LYP(6-311G(d,p)+def2tzvp(Fe only)) and measured (red) IR-spectrum of complex $\mathbf{5}$. 


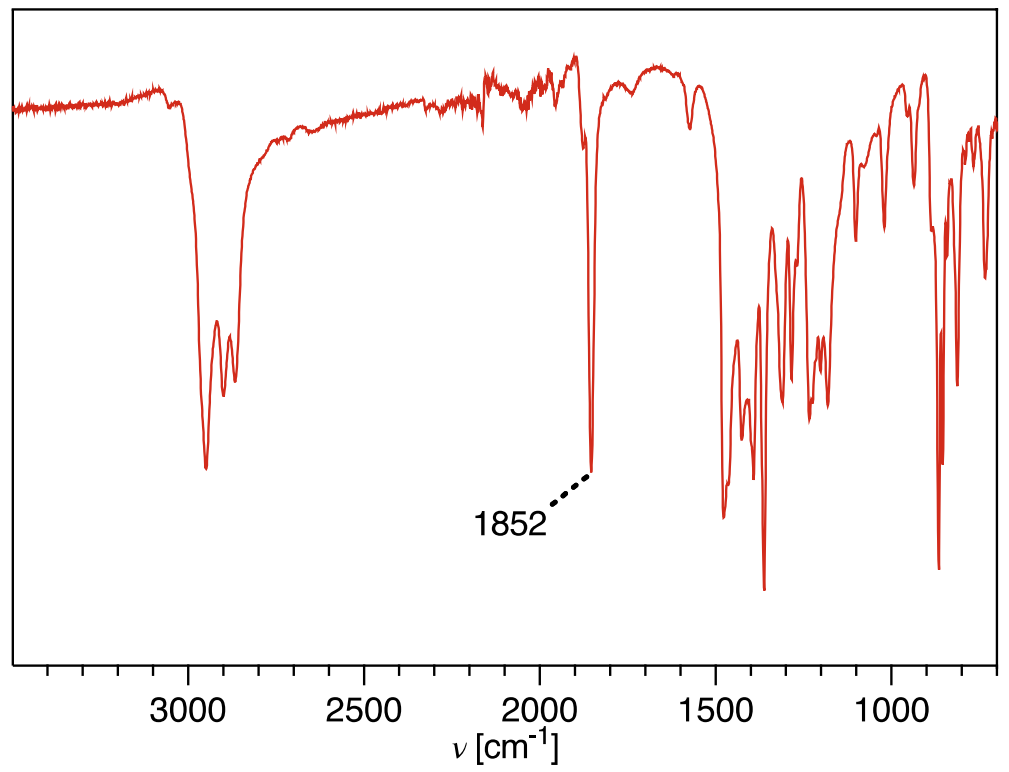

Figure S32. Powder ATR-IR spectrum of complex 6. Numbers indicate peak positions in $\mathrm{cm}^{-1}$. 


\section{S5 EPR SPECTROSCOPY}

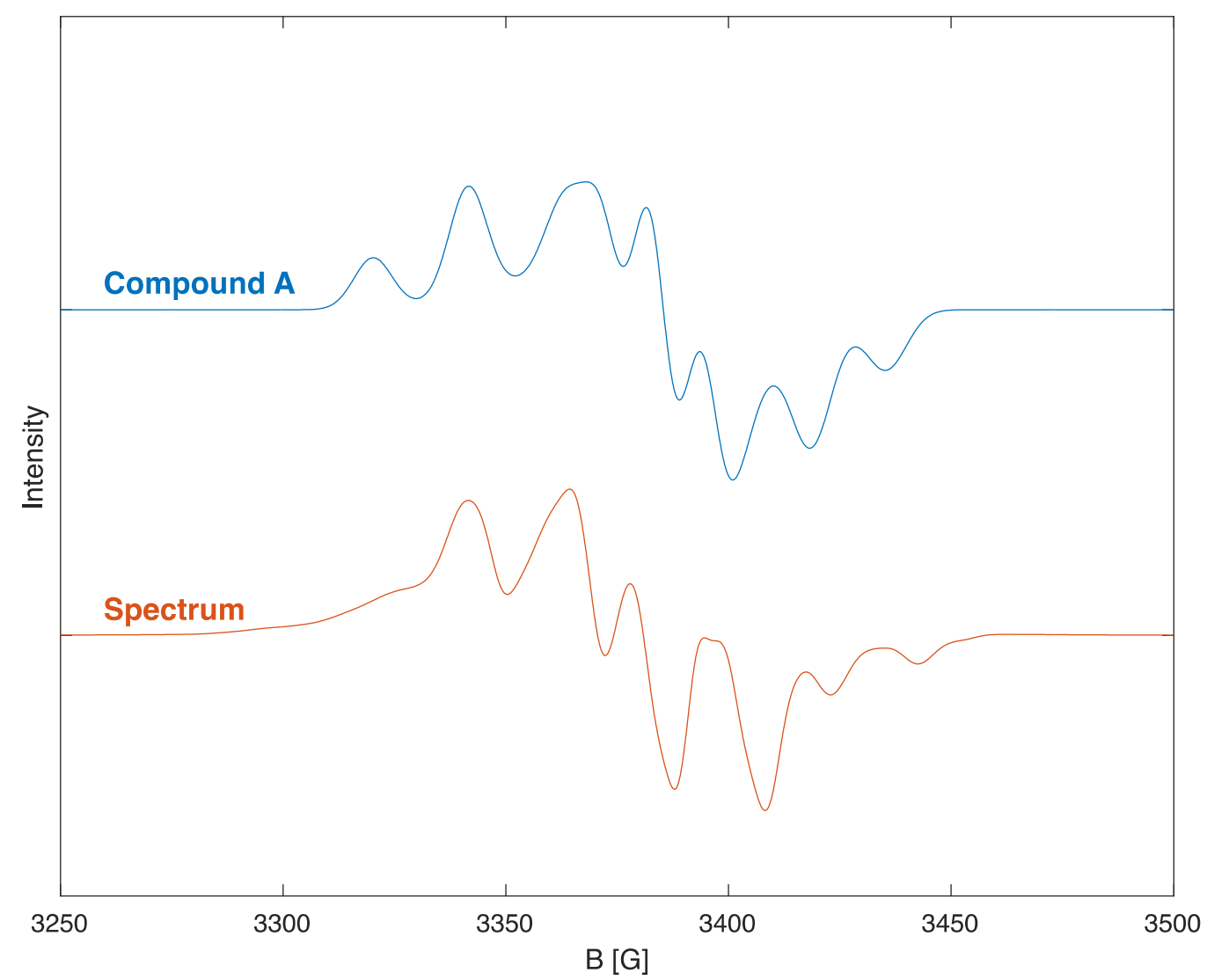

Figure S33. X-band EPR spectrum of complex $\mathbf{3}$ at $6.2 \mathrm{~K}$ in toluene glass (microwave frequency $9.633705 \mathrm{MHz}$ ). Simulation was performed with the Matlab software package Easyspin ${ }^{6}$ using the following parameters: Compound A: S [0.5], g [2.0599, 2.0332, 2.0133], A(P,P) [61.5, 32.5, 47.5; 61.5, 32.5, 47.5], HStrain $[29,23,30]$. 


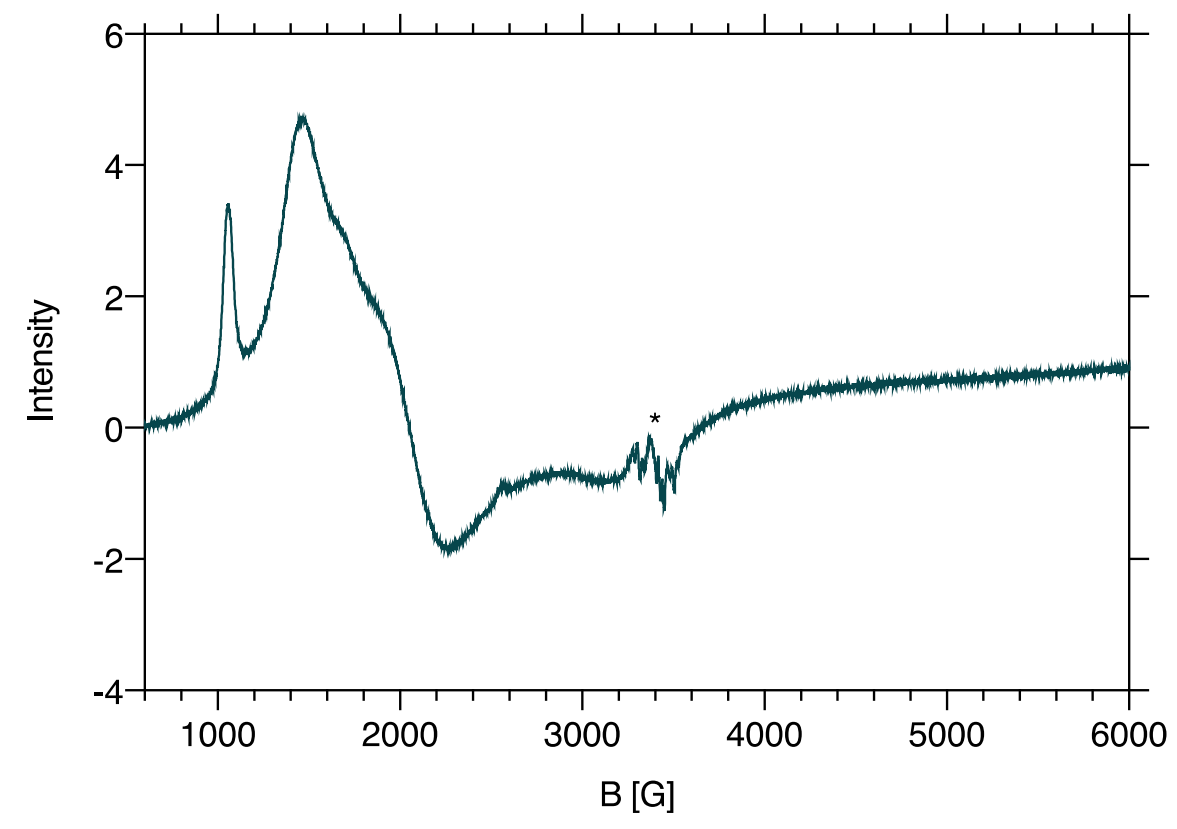

Figure S34. X-band EPR spectrum of complex 5 at $6.1 \mathrm{~K}$ in toluene glass (microwave frequency $9.632203 \mathrm{MHz}$ ). Unidentified minor paramagnetic impurity is indicated by an asterisk. 


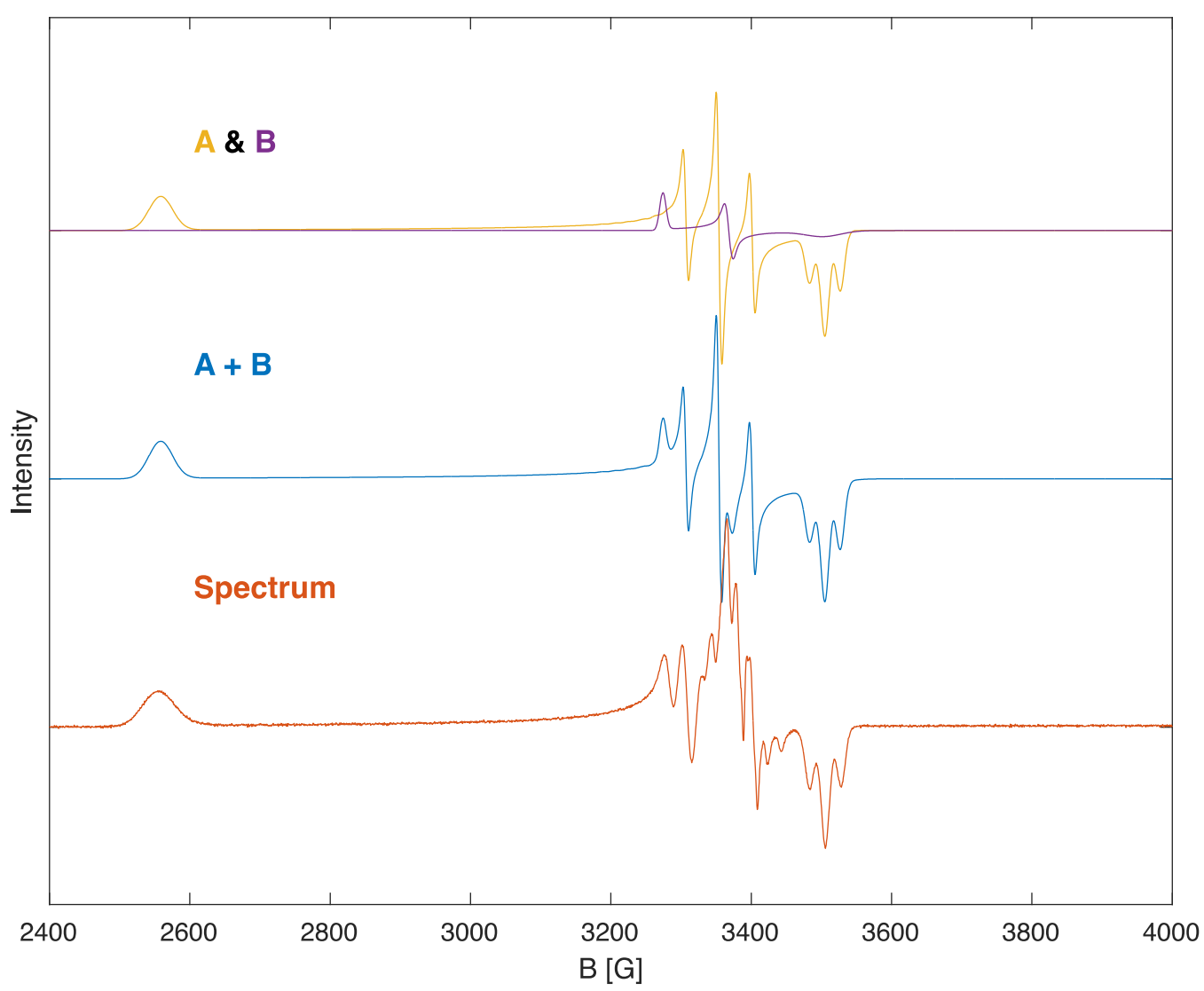

Figure S35. X-band EPR spectrum of complex 6 at $6.3 \mathrm{~K}$ in toluene glass (microwave frequency $9.633705 \mathrm{MHz}$ ). Simulation was performed with the Matlab software package Easyspin ${ }^{6}$ using the following parameters: Compound A: S [0.5], g [2.69, 2.052, 1.9635], A(P,P) [0, 136, 0; 0, 136, 0], HStrain [150, 20 , 39]; Compound B: S [0.5], g [2.1018, 2.0429, 1.9635], HStrain [30, 30, 160]; Ratio(A/B) [1/18]. 


\section{S6 Density FunCtional Theory CalCUlations}

\section{S6.1 Computational Details}

All calculations were conducted at the density functional theory (DFT) level of theory, using the $\mathrm{B} 3 \mathrm{LYP}^{7,8}$ exchange-correlation functional along with the def2-TZVP ${ }^{9,10}$ basis set for all atoms (except for complexes 7, 8 and $\mathbf{9}$, for which the 6-311G $(\mathrm{d}, \mathrm{p})^{11-13}$ basis set was used for all atoms due to the size of the systems). All calculations were executed with the Gaussian 16 Rev. C.01 software package. ${ }^{14}$ The geometries of the molecules were optimized at the same level of theory with no symmetry restrictions and the convergence criteria 'tight'. The molecular structures of the molecules obtained from X-ray crystallography were used as initial guesses for the calculations. After convergence was achieved, frequency calculations (excluding Raman vibrations) were conducted in order to establish the structures as stationary points on the potential energy surface, showing no imaginary frequencies. The stability of the wavefunctions was furthermore confirmed by an additional single-point calculation with the 'stable' keyword.

Broken-symmetry (BS)-DFT calculations were conducted for the antiferromagnetically coupled complexes $\mathbf{8}$ and $\mathbf{9}$ in order to establish an antiferromagnetically coupled ground state. The molecules were first optimized in the high-spin state, which was then used to create an initial guess for the antiferromagnetically coupled singlet. The distribution of alpha and beta spin is accounted for by the prefix in the Mulliken spin density distribution $\rho_{\mathrm{M}}$ (Table S5). After the antiferromagnetic guess was generated, the geometry of the structure was optimized akin to the high-spin isomer with the same convergence criteria and the stability of the wave function was again validated.

Table S5. Mulliken spin densities of the complexes $\mathbf{8}$ and $\mathbf{9}$ for the nonet (9et) and the antiferromagnetically coupled singlet (1et) spin isomers.

\begin{tabular}{crrrr}
\hline$\#$ & $\rho_{\mathrm{M}}(\mathbf{8}, 9 \mathrm{et})$ & $\rho_{\mathrm{M}}(\mathbf{8}, 1 \mathrm{et})$ & $\rho_{\mathrm{M}}(\mathbf{9}, 9 \mathrm{et})$ & $\rho_{\mathrm{M}}(\mathbf{9}, 1 \mathrm{et})$ \\
\hline $\mathrm{Fe}(1)$ & 3.63 & -3.52 & 3.64 & 3.55 \\
$\mathrm{Fe}(2)$ & 3.65 & 3.53 & 3.65 & -3.55 \\
\hline
\end{tabular}

The standard notation BS(n,m) for the broken-symmetry isomers represent the $(m+n)$ unpaired spins of the system with alpha and beta spin, respectively. ${ }^{15}$ For the system investigated in this study, two antiferromagnetically coupled, high-spin Fe(II) centers were established for complexes 8 and 9. The exchange coupling constant $J_{\mathrm{AFC}}$ was calculated with Equation S6:16

$$
J_{A F C}=\frac{E_{h s}-E_{b s}}{S^{2}}
$$

, where $E_{\text {hs }}$ is the energy of the high-spin isomer, $E_{\text {bs }}$ is the energy of the singlet isomer and $S$ is the total spin of the system (Table S6). 
Table S6. Summary of the BS-DFT calculations for complexes 8 and 9.

\begin{tabular}{rrrrr}
\hline$\#$ & BS description & $E_{\mathrm{hs}}[$ Hartree $]$ & $E_{\mathrm{bs}}[$ Hartree $]$ & $J_{\mathrm{AFC}}\left[\mathrm{cm}^{-1}\right]$ \\
\hline $\mathbf{8}$ & $\mathrm{BS}(4,4)$ & -7372.5760739 & -7372.5811152 & -69.2 \\
$\mathbf{9}$ & $\mathrm{BS}(4,4)$ & -9375.9144014 & -9375.9188139 & -60.5 \\
\hline
\end{tabular}
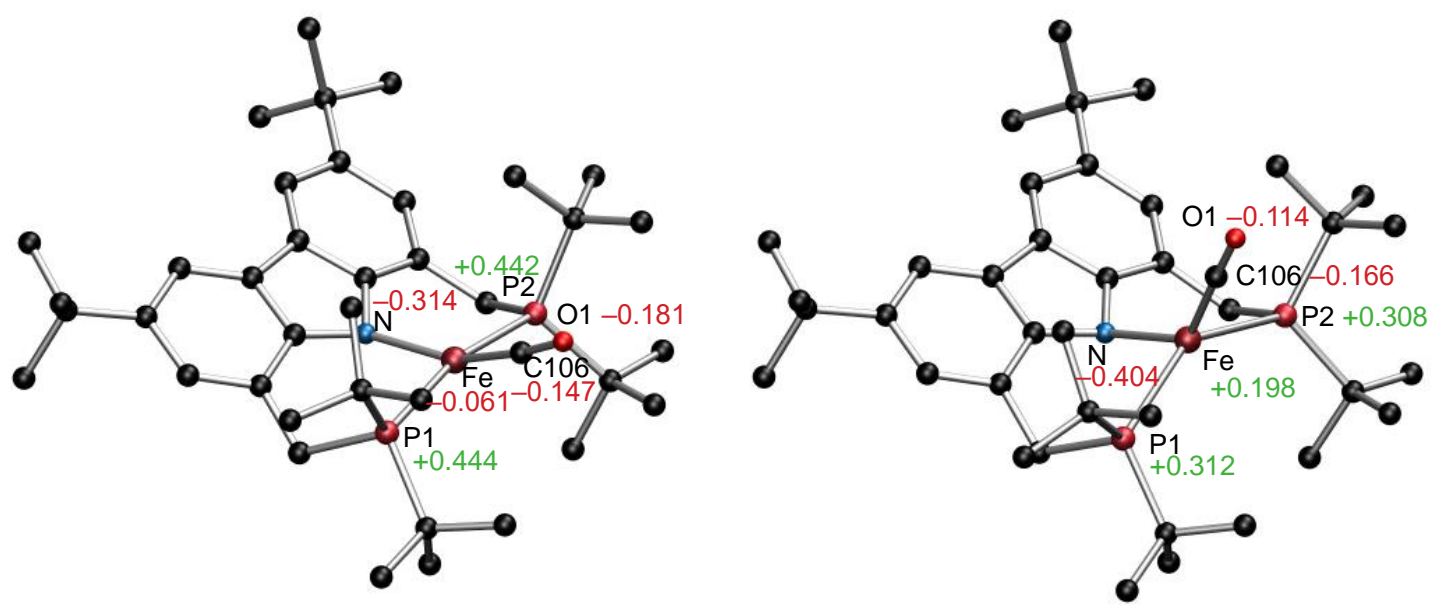

Figure S36. Molecular structures of the DFT-optimized doublet (left, E(B3LYP) $\left.=-3600.7002086 E_{\mathrm{h}}\right)$ and quartet (right, $\left.\mathrm{E}(\mathrm{B} 3 \mathrm{LYP})=-3600.696691 E_{\mathrm{h}}\right)$ spin isomers of complex 6. Numbers indicate the calculated Mulliken charge.

\section{S6.2 COORDINATES OF DFT-OPTIMIZED STRUCTURES}

$\begin{array}{lrlllllr}\left.{ }^{[\mathbf{t B u}}(\mathbf{P N P}) \mathbf{F e}(\mathbf{T E M P O})\right] \mathbf{( 2 )}, \mathbf{5 e t} & & \mathrm{C} & 4.29136700 & -1.02988800 & -0.73455400 \\ \mathrm{Fe} & -0.99722700 & -0.28246000 & 0.18546100 & \mathrm{H} & 5.15478200 & -0.54626000 & -1.17001700 \\ \mathrm{P} & -0.79172800 & -2.74056300 & 0.82766400 & \mathrm{C} & 4.30156800 & -2.39108400 & -0.46127200 \\ \mathrm{P} & -1.18849000 & 1.76143000 & 1.90526700 & \mathrm{C} & 3.16919100 & -2.93688100 & 0.18002000 \\ \mathrm{O} & -2.22422100 & -0.33144000 & -1.23062200 & \mathrm{H} & 3.19742900 & -3.97646800 & 0.48542100 \\ \mathrm{~N} & 0.99695100 & 0.08311000 & 0.31221300 & \mathrm{C} & 2.02891600 & -2.20928500 & 0.49744700 \\ \mathrm{~N} & -3.08512200 & 0.51651200 & -1.97582600 & \mathrm{C} & 2.00085200 & -0.85396400 & 0.11791300 \\ \mathrm{C} & 1.57420100 & 1.31020400 & 0.00947700 & \mathrm{C} & 0.97663900 & -2.81420400 & 1.39656300 \\ \mathrm{C} & 1.06050700 & 2.60349200 & 0.21337100 & \mathrm{H} & 0.98257500 & -2.25062600 & 2.33193800 \\ \mathrm{C} & 1.88678500 & 3.67243200 & -0.11928500 & \mathrm{H} & 1.25147600 & -3.83921800 & 1.64727900 \\ \mathrm{H} & 1.49316800 & 4.66940200 & 0.04235400 & \mathrm{C} & -0.33662400 & 2.91211400 & 0.70917400 \\ \mathrm{C} & 3.18648400 & 3.53880900 & -0.64719100 & \mathrm{H} & -1.00861200 & 2.90922600 & -0.15013400 \\ \mathrm{C} & 3.68245200 & 2.25307100 & -0.81822100 & \mathrm{H} & -0.35504900 & 3.93003200 & 1.10324300 \\ \mathrm{H} & 4.68362000 & 2.08978000 & -1.19232100 & \mathrm{C} & 3.99551100 & 4.80013800 & -0.98973100 \\ \mathrm{C} & 2.89716800 & 1.15122400 & -0.47744700 & \mathrm{C} & 5.38594400 & 4.46442300 & -1.54943600 \\ \mathrm{C} & 3.16549700 & -0.26194200 & -0.42869700 & \mathrm{H} & 5.98986700 & 3.90855900 & -0.82965200 \\ & & & & \mathrm{H} & 5.91981200 & 5.38752400 & -1.78477600\end{array}$




\begin{tabular}{|c|c|c|c|c|c|c|c|}
\hline $\mathrm{H}$ & 5.32202900 & 3.87665800 & -2.46713000 & $\mathrm{C}$ & -0.35410500 & -3.23890700 & -1.85504200 \\
\hline $\mathrm{C}$ & 4.18875100 & 5.65998600 & 0.27695900 & $\mathrm{H}$ & -0.98335400 & -2.37741000 & -2.07349200 \\
\hline $\mathrm{H}$ & 3.23481100 & 5.97416600 & 0.70259700 & $\mathrm{H}$ & -0.38069300 & -3.91346300 & -2.71617400 \\
\hline $\mathrm{H}$ & 4.76162700 & 6.56143100 & 0.04378000 & $\mathrm{H}$ & 0.67331000 & -2.89586100 & -1.73484000 \\
\hline $\mathrm{H}$ & 4.72930900 & 5.10302000 & 1.04499700 & $\mathrm{C}$ & -0.19361400 & 1.92629900 & 3.54234400 \\
\hline $\mathrm{C}$ & 3.24354000 & 5.62805300 & -2.05310700 & $\mathrm{C}$ & -1.04707500 & 1.48202600 & 4.74173800 \\
\hline $\mathrm{H}$ & 3.10201500 & 5.04791900 & -2.96715700 & $\mathrm{H}$ & -1.86632800 & 2.16497300 & 4.95789300 \\
\hline $\mathrm{H}$ & 3.80801800 & 6.52956300 & -2.30582000 & $\mathrm{H}$ & -0.41110200 & 1.44688100 & 5.63084500 \\
\hline $\mathrm{H}$ & 2.25898700 & 5.93942700 & -1.70167800 & $\mathrm{H}$ & -1.46299900 & 0.48346800 & 4.60097700 \\
\hline $\mathrm{C}$ & 5.49883500 & -3.29972300 & -0.78286500 & $\mathrm{C}$ & 1.01630700 & 0.97759500 & 3.46350600 \\
\hline $\mathrm{C}$ & 5.04831700 & -4.43814200 & -1.72207200 & $\mathrm{H}$ & 0.71094100 & -0.05750900 & 3.31989500 \\
\hline $\mathrm{H}$ & 4.26701200 & -5.05009000 & -1.26918600 & $\mathrm{H}$ & 1.56700700 & 1.03906100 & 4.40704100 \\
\hline $\mathrm{H}$ & 5.88978200 & -5.09435500 & -1.95979600 & $\mathrm{H}$ & 1.69859500 & 1.23977200 & 2.65873700 \\
\hline $\mathrm{H}$ & 4.65565800 & -4.03618200 & -2.65824600 & $\mathrm{C}$ & 0.33960800 & 3.34754500 & 3.78575100 \\
\hline $\mathrm{C}$ & 6.06240800 & -3.91049400 & 0.51755100 & $\mathrm{H}$ & 1.02824800 & 3.65861400 & 3.00050800 \\
\hline $\mathrm{H}$ & 6.39802500 & -3.12710900 & 1.20004300 & $\mathrm{H}$ & 0.89763900 & 3.35770700 & 4.72714900 \\
\hline $\mathrm{H}$ & 6.91501000 & -4.55838400 & 0.29743500 & $\mathrm{H}$ & -0.44971900 & 4.09172500 & 3.86804800 \\
\hline $\mathrm{H}$ & 5.31678200 & -4.51074500 & 1.04068700 & $\mathrm{C}$ & -2.89765800 & 2.60815400 & 2.09352300 \\
\hline $\mathrm{C}$ & 6.63659400 & -2.53708500 & -1.47799200 & $\mathrm{C}$ & -3.85259600 & 1.59191700 & 2.74703900 \\
\hline $\mathrm{H}$ & 6.30961500 & -2.09370800 & -2.42043800 & $\mathrm{H}$ & -3.91711200 & 0.67603200 & 2.15911600 \\
\hline $\mathrm{H}$ & 7.45605800 & -3.22342900 & -1.70145100 & $\mathrm{H}$ & -4.85647000 & 2.02294900 & 2.80220200 \\
\hline $\mathrm{H}$ & 7.03686500 & -1.74106800 & -0.84723900 & $\mathrm{H}$ & -3.55531400 & 1.32420100 & 3.75963900 \\
\hline $\mathrm{C}$ & -1.75867300 & -3.39002900 & 2.34581900 & $\mathrm{C}$ & -2.91754300 & 3.92066000 & 2.89110300 \\
\hline $\mathrm{C}$ & -1.34269200 & -2.53240400 & 3.55460100 & $\mathrm{H}$ & -2.67719900 & 3.78523800 & 3.94404800 \\
\hline $\mathrm{H}$ & -0.33476000 & -2.75848000 & 3.90153800 & $\mathrm{H}$ & -3.92464900 & 4.34635400 & 2.84244400 \\
\hline $\mathrm{H}$ & -2.02562400 & -2.73439100 & 4.38432600 & $\mathrm{H}$ & -2.23536800 & 4.66370200 & 2.47598900 \\
\hline $\mathrm{H}$ & -1.40078700 & -1.46667200 & 3.32994700 & $\mathrm{C}$ & -3.42691900 & 2.91413900 & 0.67917200 \\
\hline $\mathrm{C}$ & -3.26101600 & -3.14862800 & 2.11329800 & $\mathrm{H}$ & -2.94327200 & 3.79146600 & 0.24882700 \\
\hline $\mathrm{H}$ & -3.46615600 & -2.09748500 & 1.90697600 & $\mathrm{H}$ & -4.49491100 & 3.14051300 & 0.74962000 \\
\hline $\mathrm{H}$ & -3.81265500 & -3.42479900 & 3.01669500 & $\mathrm{H}$ & -3.31081800 & 2.08493100 & -0.02069300 \\
\hline $\mathrm{H}$ & -3.66258400 & -3.73689500 & 1.29161000 & $\mathrm{C}$ & -2.40008100 & 0.99803200 & -3.21650700 \\
\hline $\mathrm{C}$ & -1.51759900 & -4.86604200 & 2.69196100 & $\mathrm{C}$ & -3.39254400 & 1.90265700 & -3.97607700 \\
\hline $\mathrm{H}$ & -1.90140900 & -5.54041400 & 1.92806900 & $\mathrm{H}$ & -3.54523300 & 2.81205900 & -3.38649700 \\
\hline $\mathrm{H}$ & -2.03658100 & -5.10556500 & 3.62527800 & $\mathrm{H}$ & -2.92397600 & 2.21000800 & -4.91485000 \\
\hline $\mathrm{H}$ & -0.46012600 & -5.08688200 & 2.84454900 & $\mathrm{C}$ & -4.74696500 & 1.25499700 & -4.23087300 \\
\hline $\mathrm{C}$ & -0.86067400 & -3.99615500 & -0.61253500 & $\mathrm{H}$ & -4.64958200 & 0.41984800 & -4.92943700 \\
\hline $\mathrm{C}$ & -2.30473500 & -4.44639100 & -0.87996100 & $\mathrm{H}$ & -5.41994800 & 1.97209700 & -4.70910600 \\
\hline $\mathrm{H}$ & -2.67985000 & -5.12153500 & -0.11116900 & $\mathrm{C}$ & -5.33979500 & 0.78433500 & -2.90905000 \\
\hline $\mathrm{H}$ & -2.33386900 & -4.98875500 & -1.82923400 & $\mathrm{H}$ & -6.29375400 & 0.27369100 & -3.06798300 \\
\hline $\mathrm{H}$ & -2.98844300 & -3.60301700 & -0.96501100 & $\mathrm{H}$ & -5.54775500 & 1.65844700 & -2.28404700 \\
\hline $\mathrm{C}$ & 0.02332600 & -5.23610800 & -0.40418000 & $\mathrm{C}$ & -4.40984600 & -0.16241400 & -2.12650400 \\
\hline $\mathrm{H}$ & 1.07887400 & -4.97654000 & -0.34078400 & $\mathrm{C}$ & -1.84544100 & -0.10608000 & -4.14073900 \\
\hline $\mathrm{H}$ & -0.09355100 & -5.89605700 & -1.26901300 & $\mathrm{H}$ & -1.26242400 & -0.82186500 & -3.56484400 \\
\hline $\mathrm{H}$ & -0.24614800 & -5.81014100 & 0.48091700 & $\mathrm{H}$ & -1.18360200 & 0.34198400 & -4.88498500 \\
\hline
\end{tabular}




\begin{tabular}{|c|c|c|c|c|c|c|c|}
\hline $\mathrm{H}$ & -2.62076000 & -0.64740300 & -4.67748100 & C & 1.89641700 & 5.76302400 & 0.21350400 \\
\hline $\mathrm{C}$ & -1.21831900 & 1.87629000 & -2.78980400 & $\mathrm{C}$ & 1.47489900 & 6.21054800 & 1.62875100 \\
\hline $\mathrm{H}$ & -1.55861700 & 2.67422500 & -2.12999300 & $\mathrm{H}$ & 0.39239500 & 6.17406000 & 1.75606100 \\
\hline $\mathrm{H}$ & -0.76369600 & 2.33870500 & -3.66813900 & $\mathrm{H}$ & 1.80044800 & 7.23677200 & 1.81942400 \\
\hline $\mathrm{H}$ & -0.44545200 & 1.30013300 & -2.28401100 & $\mathrm{H}$ & 1.92008600 & 5.56541400 & 2.38893000 \\
\hline $\mathrm{C}$ & -4.35965700 & -1.55137500 & -2.80049000 & $\mathrm{C}$ & 3.42278400 & 5.90212700 & 0.10949900 \\
\hline $\mathrm{H}$ & -4.29993700 & -1.49730500 & -3.88527400 & $\mathrm{H}$ & 3.93815300 & 5.27144100 & 0.83673900 \\
\hline $\mathrm{H}$ & -5.26136700 & -2.11610300 & -2.55328800 & $\mathrm{H}$ & 3.71103100 & 6.93668900 & 0.30735700 \\
\hline $\mathrm{H}$ & -3.49902800 & -2.10898100 & -2.43939900 & $\mathrm{H}$ & 3.78830000 & 5.64455900 & -0.88663700 \\
\hline $\mathrm{C}$ & -4.99922400 & -0.34629000 & -0.72128200 & $\mathrm{C}$ & 1.26190600 & 6.71083000 & -0.82572500 \\
\hline $\mathrm{H}$ & -4.40826500 & -1.05013000 & -0.13841000 & $\mathrm{H}$ & 1.54989700 & 6.42449200 & -1.83934800 \\
\hline $\mathrm{H}$ & -6.01796600 & -0.73287100 & -0.79651100 & $\mathrm{H}$ & 1.58983700 & 7.74000100 & -0.65631800 \\
\hline $\mathrm{H}$ & -5.03413900 & 0.60333300 & -0.18911400 & $\mathrm{H}$ & 0.17273100 & 6.69536100 & -0.77328800 \\
\hline \multirow{3}{*}{\multicolumn{4}{|c|}{$\left[{ }^{t \mathrm{Bu}}(\mathrm{PNP}) \mathrm{Fe}(\mathrm{CO})_{2}\right](3)$, et }} & $\mathrm{C}$ & -5.51566200 & 2.47075500 & -0.46408700 \\
\hline & & & & $\mathrm{C}$ & -6.35586900 & 1.72797700 & 0.59588100 \\
\hline & & & & $\mathrm{H}$ & -6.40915800 & 0.65750400 & 0.39233900 \\
\hline $\mathrm{Fe}$ & 0.63771800 & -1.31149800 & 0.31523800 & $\mathrm{H}$ & -7.37856600 & 2.11392700 & 0.61315400 \\
\hline $\mathrm{P}$ & -1.47192800 & -2.49980200 & 0.02476000 & $\mathrm{H}$ & -5.92708800 & 1.85765500 & 1.59166000 \\
\hline $\mathrm{P}$ & 2.87622100 & -0.70585800 & -0.34878900 & $\mathrm{C}$ & -6.16340900 & 2.27384100 & -1.85080700 \\
\hline $\mathrm{O}$ & 1.65961400 & -3.85368800 & 1.30256300 & $\mathrm{H}$ & -5.59649500 & 2.79989600 & -2.62147200 \\
\hline $\mathrm{O}$ & 0.43928500 & -0.06800100 & 3.01479600 & $\mathrm{H}$ & -7.18545600 & 2.66205600 & -1.85499900 \\
\hline $\mathrm{N}$ & -0.12745700 & 0.42126600 & -0.56980800 & $\mathrm{H}$ & -6.20661100 & 1.22042000 & -2.13095600 \\
\hline $\mathrm{C}$ & 0.52827200 & 1.64802500 & -0.41831300 & $\mathrm{C}$ & -5.55487000 & 3.96979100 & -0.13151100 \\
\hline $\mathrm{C}$ & 1.88537200 & 1.96018100 & -0.56855200 & $\mathrm{H}$ & -5.13128200 & 4.17728300 & 0.85303000 \\
\hline $\mathrm{C}$ & 2.29362600 & 3.27581500 & -0.35374400 & $\mathrm{H}$ & -6.58978400 & 4.31838000 & -0.12664800 \\
\hline $\mathrm{H}$ & 3.34700600 & 3.48914200 & -0.47931300 & $\mathrm{H}$ & -5.01065000 & 4.56300900 & -0.86875900 \\
\hline $\mathrm{C}$ & 1.41642700 & 4.32314100 & -0.03316100 & $\mathrm{C}$ & -1.45313000 & -4.19074500 & -0.92215700 \\
\hline $\mathrm{C}$ & 0.05925000 & 4.01848400 & 0.02091600 & $\mathrm{C}$ & -0.35428800 & -4.09938500 & -1.99222200 \\
\hline $\mathrm{H}$ & -0.67165000 & 4.79600500 & 0.20832800 & $\mathrm{H}$ & -0.48858000 & -3.24445700 & -2.65610700 \\
\hline $\mathrm{C}$ & -0.37818400 & 2.71381800 & -0.18136700 & $\mathrm{H}$ & -0.38462600 & -5.00017400 & -2.61207400 \\
\hline $\mathrm{C}$ & -1.68784600 & 2.13684100 & -0.28209400 & $\mathrm{H}$ & 0.63671800 & -4.03811600 & -1.54822200 \\
\hline $\mathrm{C}$ & -2.96218100 & 2.70155500 & -0.23994400 & $\mathrm{C}$ & -1.12032700 & -5.38520600 & -0.01173300 \\
\hline $\mathrm{H}$ & -3.05819200 & 3.76014400 & -0.04155500 & $\mathrm{H}$ & -0.20414400 & -5.24034900 & 0.55629600 \\
\hline $\mathrm{C}$ & -4.08276500 & 1.91623400 & -0.47585800 & $\mathrm{H}$ & -0.97759100 & -6.26850700 & -0.64134700 \\
\hline $\mathrm{C}$ & -3.86334700 & 0.55509100 & -0.75629200 & $\mathrm{H}$ & -1.92537300 & -5.61664100 & 0.68326500 \\
\hline $\mathrm{H}$ & -4.71689500 & -0.07935800 & -0.96742000 & $\mathrm{C}$ & -2.77669400 & -4.49148900 & -1.65328500 \\
\hline $\mathrm{C}$ & -2.60778300 & -0.04095800 & -0.79030000 & $\mathrm{H}$ & -3.63806600 & -4.52311600 & -0.99100800 \\
\hline $\mathrm{C}$ & -1.48250800 & 0.75842400 & -0.53811000 & $\mathrm{H}$ & -2.69158700 & -5.47538500 & -2.12355300 \\
\hline $\mathrm{C}$ & -2.49260000 & -1.49077600 & -1.14323200 & $\mathrm{H}$ & -2.98244500 & -3.77534100 & -2.44767000 \\
\hline $\mathrm{H}$ & -1.97809900 & -1.59975000 & -2.10384600 & $\mathrm{C}$ & -2.51740700 & -2.70235400 & 1.62413200 \\
\hline $\mathrm{H}$ & -3.48560300 & -1.92352500 & -1.25942400 & $\mathrm{C}$ & -1.67439800 & -3.40491400 & 2.70421900 \\
\hline $\mathrm{C}$ & 2.86181400 & 0.97001900 & -1.12637400 & $\mathrm{H}$ & -1.37306800 & -4.41256200 & 2.42823100 \\
\hline $\mathrm{H}$ & 3.86849500 & 1.39017700 & -1.13041500 & $\mathrm{H}$ & -2.27179500 & -3.47850800 & 3.61721200 \\
\hline $\mathrm{H}$ & 2.59636500 & 0.78453500 & -2.16997100 & $\mathrm{H}$ & -0.77768800 & -2.83754500 & 2.94761100 \\
\hline
\end{tabular}




\begin{tabular}{|c|c|c|c|c|c|c|c|}
\hline $\mathrm{C}$ & -3.82804800 & -3.47858000 & 1.41728300 & $\mathrm{C}$ & 2.13850000 & 1.18828100 & -0.40753900 \\
\hline $\mathrm{H}$ & -4.45253800 & -3.03556800 & 0.64049800 & $\mathrm{C}$ & 2.93868500 & 2.33274700 & -0.41602300 \\
\hline $\mathrm{H}$ & -4.40182000 & -3.44352400 & 2.34785600 & $\mathrm{H}$ & 3.99972800 & 2.18533300 & -0.56513400 \\
\hline $\mathrm{H}$ & -3.66888600 & -4.52819300 & 1.17808400 & $\mathrm{C}$ & 2.44986400 & 3.63954100 & -0.25080400 \\
\hline $\mathrm{C}$ & -2.89238600 & -1.30697600 & 2.15984900 & $\mathrm{C}$ & 1.07448800 & 3.80052400 & -0.09931100 \\
\hline $\mathrm{H}$ & -2.03526300 & -0.64714300 & 2.26297500 & $\mathrm{H}$ & 0.64421300 & 4.79033900 & -0.00485300 \\
\hline $\mathrm{H}$ & -3.33166300 & -1.43343200 & 3.15362200 & $\mathrm{C}$ & 0.23549700 & 2.69112200 & -0.10028700 \\
\hline $\mathrm{H}$ & -3.62441800 & -0.80626700 & 1.53169900 & $\mathrm{C}$ & -1.19794800 & 2.54236100 & -0.08383000 \\
\hline $\mathrm{C}$ & 3.59289500 & -1.74460800 & -1.80145900 & $\mathrm{C}$ & -2.25470300 & 3.45406100 & -0.03722600 \\
\hline $\mathrm{C}$ & 2.45286800 & -1.87158500 & -2.82918600 & $\mathrm{H}$ & -2.03692200 & 4.50621400 & 0.08382000 \\
\hline $\mathrm{H}$ & 2.76143100 & -2.55952300 & -3.62150000 & $\mathrm{C}$ & -3.56475400 & 3.01029600 & -0.16685500 \\
\hline $\mathrm{H}$ & 2.21197600 & -0.91870400 & -3.30104200 & $\mathrm{C}$ & -3.76983400 & 1.63320900 & -0.39852500 \\
\hline $\mathrm{C}$ & 3.99081500 & -3.15140700 & -1.32950900 & $\mathrm{H}$ & -4.77800800 & 1.28327700 & -0.58896100 \\
\hline $\mathrm{H}$ & 4.83881700 & -3.13218600 & -0.64525000 & $\mathrm{C}$ & -2.74735400 & 0.69424400 & -0.44917000 \\
\hline $\mathrm{H}$ & 4.28876300 & -3.74738900 & -2.19703500 & $\mathrm{C}$ & -1.43754400 & 1.15392900 & -0.21911600 \\
\hline $\mathrm{H}$ & 3.17327900 & -3.67549000 & -0.83758400 & $\mathrm{C}$ & -3.02619500 & -0.71220600 & -0.92395100 \\
\hline $\mathrm{C}$ & 4.79223900 & -1.11132000 & -2.53172500 & $\mathrm{H}$ & -2.53702700 & -0.83596800 & -1.89270900 \\
\hline $\mathrm{H}$ & 4.58564300 & -0.09875700 & -2.87677800 & $\mathrm{H}$ & -4.09558400 & -0.84277300 & -1.09150600 \\
\hline $\mathrm{H}$ & 5.00870400 & -1.71317500 & -3.41946100 & $\mathrm{C}$ & 2.81730100 & -0.15733200 & -0.56582600 \\
\hline $\mathrm{H}$ & 5.69634800 & -1.08980700 & -1.93051900 & $\mathrm{H}$ & 2.99894200 & -0.56957600 & 0.42888800 \\
\hline $\mathrm{C}$ & 4.17214000 & -0.53995500 & 1.06169200 & $\mathrm{H}$ & 3.79933500 & -0.00198800 & -1.01647900 \\
\hline $\mathrm{C}$ & 4.10985200 & -1.74999100 & 2.00756100 & $\mathrm{C}$ & 3.37013400 & 4.87266400 & -0.25994700 \\
\hline $\mathrm{H}$ & 3.14750400 & -1.82282300 & 2.50993300 & $\mathrm{C}$ & 3.22403300 & 5.63620400 & 1.07291400 \\
\hline $\mathrm{H}$ & 4.87342900 & -1.62838800 & 2.78112400 & $\mathrm{H}$ & 2.19744300 & 5.96468100 & 1.23894300 \\
\hline $\mathrm{H}$ & 4.30120700 & -2.69607000 & 1.50346200 & $\mathrm{H}$ & 3.86413500 & 6.52252700 & 1.07919000 \\
\hline $\mathrm{C}$ & 5.61856100 & -0.38242200 & 0.56774400 & $\mathrm{H}$ & 3.51108600 & 5.00393000 & 1.91569700 \\
\hline $\mathrm{H}$ & 6.01160800 & -1.29899800 & 0.13056400 & $\mathrm{C}$ & 4.85174500 & 4.50474000 & -0.43196100 \\
\hline $\mathrm{H}$ & 6.25165300 & -0.13798400 & 1.42555300 & $\mathrm{H}$ & 5.20699900 & 3.86030100 & 0.37456100 \\
\hline $\mathrm{H}$ & 5.73232600 & 0.42489700 & -0.15602000 & $\mathrm{H}$ & 5.45807700 & 5.41291800 & -0.42013800 \\
\hline $\mathrm{C}$ & 3.81877400 & 0.72029300 & 1.87487700 & $\mathrm{H}$ & 5.03808500 & 3.99818100 & -1.38102900 \\
\hline $\mathrm{H}$ & 3.98702100 & 1.63652700 & 1.31230300 & $\mathrm{C}$ & 2.97625500 & 5.80759200 & -1.42279200 \\
\hline $\mathrm{H}$ & 4.46385100 & 0.75023400 & 2.75771700 & $\mathrm{H}$ & 3.07675000 & 5.29657200 & -2.38253900 \\
\hline $\mathrm{H}$ & 2.78843800 & 0.72673600 & 2.22162300 & $\mathrm{H}$ & 3.61955600 & 6.69151300 & -1.43879200 \\
\hline $\mathrm{C}$ & 1.26994700 & -2.84932400 & 0.88533000 & $\mathrm{H}$ & 1.94395900 & 6.14811700 & -1.33465400 \\
\hline $\mathrm{C}$ & 0.52076900 & -0.57217500 & 1.98860900 & $\mathrm{C}$ & -4.77616000 & 3.95511200 & -0.11459800 \\
\hline $\mathrm{H}$ & 1.54201900 & -2.25791700 & -2.38167300 & $\mathrm{C}$ & -5.72812800 & 3.51714400 & 1.01817900 \\
\hline \multirow{3}{*}{\multicolumn{4}{|c|}{${ }^{t \mathrm{Bu}}(\mathrm{PNP}) \mathrm{Fe}(\mathrm{HCCPh})(4)$, 4et }} & $\mathrm{H}$ & -6.09405900 & 2.50050200 & 0.86808000 \\
\hline & & & & $\mathrm{H}$ & -6.59624700 & 4.17987400 & 1.06677600 \\
\hline & & & & $\mathrm{H}$ & -5.22134300 & 3.54934800 & 1.98480800 \\
\hline $\mathrm{Fe}$ & -0.02594400 & -1.42768200 & 0.50717100 & $\mathrm{C}$ & -5.53562000 & 3.91323400 & -1.45747500 \\
\hline $\mathrm{P}$ & -2.34037300 & -2.09739100 & 0.11012000 & $\mathrm{H}$ & -4.88860900 & 4.22860500 & -2.27846900 \\
\hline $\mathrm{P}$ & 1.92139400 & -1.54156100 & -1.44847300 & $\mathrm{H}$ & -6.40015000 & 4.58183200 & -1.42920800 \\
\hline $\mathrm{N}$ & -0.25809600 & 0.42877700 & -0.21868400 & $\mathrm{H}$ & -5.89935300 & 2.91073000 & -1.68679900 \\
\hline $\mathrm{C}$ & 0.76031900 & 1.37401200 & -0.21630200 & $\mathrm{C}$ & -4.36661000 & 5.41155000 & 0.15073600 \\
\hline
\end{tabular}




$\begin{array}{lrrrlrrr}\mathrm{H} & -3.83885900 & 5.51686100 & 1.10056200 & \mathrm{H} & 2.48317500 & -2.72322600 & -4.33499100 \\ \mathrm{H} & -5.25762800 & 6.04134100 & 0.19523500 & \mathrm{H} & 1.34718700 & -1.72342800 & -5.22663000 \\ \mathrm{H} & -3.72568300 & 5.80396800 & -0.64103500 & \mathrm{H} & 0.76332300 & -2.76161700 & -3.93132000 \\ \mathrm{C} & -2.70759600 & -3.65394000 & -0.93986600 & \mathrm{C} & 0.49833200 & -0.06682100 & -3.37048600 \\ \mathrm{C} & -1.98749300 & -4.85880700 & -0.30759600 & \mathrm{H} & -0.38979700 & -0.62263900 & -3.07284400 \\ \mathrm{H} & -0.92197300 & -4.66216900 & -0.18560500 & \mathrm{H} & 0.36916700 & 0.24417800 & -4.41176900 \\ \mathrm{H} & -2.09774000 & -5.72652600 & -0.96448900 & \mathrm{H} & 0.55332700 & 0.82918600 & -2.75782700 \\ \mathrm{H} & -2.39456400 & -5.13125000 & 0.66394600 & \mathrm{C} & 2.95886500 & -0.06946100 & -3.73284100 \\ \mathrm{C} & -4.19499600 & -3.98129700 & -1.13141700 & \mathrm{H} & 3.05830100 & 0.83508300 & -3.13346200 \\ \mathrm{H} & -4.67909100 & -4.27616200 & -0.20149000 & \mathrm{H} & 2.78699500 & 0.24519600 & -4.76722000 \\ \mathrm{H} & -4.29139700 & -4.82123000 & -1.82646000 & \mathrm{H} & 3.90641300 & -0.60397400 & -3.70919400 \\ \mathrm{H} & -4.75043600 & -3.14435800 & -1.55711200 & \mathrm{C} & 0.24120500 & -2.75960200 & 1.95020000 \\ \mathrm{C} & -2.07601300 & -3.42089500 & -2.32520000 & \mathrm{C} & 1.05279000 & -1.79593900 & 2.15358500 \\ \mathrm{H} & -2.62675200 & -2.69004800 & -2.91702800 & \mathrm{C} & 2.07509700 & -1.24906800 & 3.02533700 \\ \mathrm{H} & -2.08470100 & -4.36261200 & -2.88088700 & \mathrm{C} & 2.88770600 & -2.10271000 & 3.78904600 \\ \mathrm{H} & -1.03923700 & -3.09150200 & -2.24386400 & \mathrm{H} & 2.75038000 & -3.17335100 & 3.70615900 \\ \mathrm{C} & -3.44396700 & -2.05786400 & 1.68148400 & \mathrm{C} & 3.85473100 & -1.58827100 & 4.63958400 \\ \mathrm{C} & -3.39858500 & -3.39396900 & 2.43962600 & \mathrm{H} & 4.47424300 & -2.26190200 & 5.21897600 \\ \mathrm{H} & -3.91435700 & -4.19388400 & 1.90936400 & \mathrm{C} & 4.02540900 & -0.21125700 & 4.75474700 \\ \mathrm{H} & -3.90194800 & -3.26709100 & 3.40239500 & \mathrm{H} & 4.77799200 & 0.18952800 & 5.42215600 \\ \mathrm{H} & -2.37934500 & -3.71689400 & 2.64719200 & \mathrm{C} & 3.22568700 & 0.64551400 & 4.00667600 \\ \mathrm{C} & -2.86414600 & -0.96647600 & 2.60419500 & \mathrm{H} & 3.35203800 & 1.71762400 & 4.08976200 \\ \mathrm{H} & -1.85269200 & -1.20562600 & 2.92918200 & \mathrm{C} & 2.26609300 & 0.13362500 & 3.14295400 \\ \mathrm{H} & -3.49361400 & -0.89112900 & 3.49611100 & \mathrm{H} & 1.65371300 & 0.80311800 & 2.55362600 \\ \mathrm{H} & -2.85083600 & 0.01210900 & 2.12609500 & \mathrm{H} & -0.15221700 & -3.67686600 & 2.35287200 \\ \mathrm{C} & -4.91191600 & -1.70275100 & 1.39071800 & & & & \end{array}$

\section{$\left[{ }^{\mathrm{BBu}}(\mathbf{P N P}) \mathrm{Fe}\left(\mathrm{N}_{2} \mathrm{CPh}_{2}\right)\right](5)$, 4et}

$\begin{array}{lrrr}\mathrm{Fe} & -0.54923400 & -0.44558400 & 0.72259600 \\ \mathrm{P} & -0.33898100 & -2.87989500 & 0.90074500 \\ \mathrm{P} & -0.35854700 & 1.46855900 & 2.45883100 \\ \mathrm{~N} & 1.37479700 & -0.11231200 & 0.27512400 \\ \mathrm{~N} & -2.12289800 & -0.18821200 & -0.17435300 \\ \mathrm{~N} & -3.18882800 & -0.26581200 & -0.75032100 \\ \mathrm{C} & 1.87002000 & 1.13728600 & -0.06986000 \\ \mathrm{C} & 1.39692500 & 2.40828600 & 0.30591300 \\ \mathrm{C} & 2.12616200 & 3.50798300 & -0.13542700 \\ \mathrm{H} & 1.76601600 & 4.48820900 & 0.15498100 \\ \mathrm{C} & 3.28835600 & 3.42507900 & -0.92894400 \\ \mathrm{C} & 3.74588100 & 2.15919300 & -1.27183800 \\ \mathrm{H} & 4.64520700 & 2.03509100 & -1.85902600 \\ \mathrm{C} & 3.05713600 & 1.02666100 & -0.83661200 \\ \mathrm{C} & 3.32444600 & -0.38529000 & -0.94385500 \\ \mathrm{C} & 4.34115300 & -1.12088200 & -1.54597500\end{array}$




\begin{tabular}{|c|c|c|c|c|c|c|c|}
\hline $\mathrm{H}$ & 5.10351200 & -0.60279700 & -2.11533500 & $\mathrm{H}$ & -0.25097900 & -2.05141900 & 3.64709400 \\
\hline $\mathrm{C}$ & 4.38575000 & -2.50593300 & -1.40647000 & $\mathrm{H}$ & 0.76194000 & -3.50055900 & 3.73779600 \\
\hline $\mathrm{C}$ & 3.40321600 & -3.11141400 & -0.60296400 & $\mathrm{H}$ & -0.77415500 & -3.44502700 & 4.59265700 \\
\hline $\mathrm{H}$ & 3.45738100 & -4.17630300 & -0.42090000 & $\mathrm{C}$ & -0.87601100 & -5.26076400 & 2.50103900 \\
\hline $\mathrm{C}$ & 2.37017800 & -2.41297300 & 0.02050900 & $\mathrm{H}$ & -1.19867900 & -5.63222900 & 3.47855700 \\
\hline $\mathrm{C}$ & 2.29736600 & -1.02848900 & -0.20402600 & $\mathrm{H}$ & 0.15709400 & -5.58101000 & 2.35785600 \\
\hline $\mathrm{C}$ & 1.49868400 & -3.12447500 & 1.03074000 & $\mathrm{H}$ & -1.49417000 & -5.75107300 & 1.75036500 \\
\hline $\mathrm{H}$ & 1.74993700 & -2.73432000 & 2.02001500 & $\mathrm{C}$ & -0.83783300 & -3.82148300 & -0.68561100 \\
\hline $\mathrm{H}$ & 1.74316800 & -4.18754000 & 1.04325900 & $\mathrm{C}$ & -2.35494100 & -4.06541700 & -0.71558300 \\
\hline $\mathrm{C}$ & 0.12913500 & 2.65598500 & 1.09843300 & $\mathrm{H}$ & -2.63074400 & -4.43795100 & -1.70602000 \\
\hline $\mathrm{H}$ & -0.72043200 & 2.60922400 & 0.41203900 & $\mathrm{H}$ & -2.92117800 & -3.14957000 & -0.54410000 \\
\hline $\mathrm{H}$ & 0.14977200 & 3.67539100 & 1.48841100 & $\mathrm{H}$ & -2.67101500 & -4.81492600 & 0.00974700 \\
\hline $\mathrm{C}$ & 3.99871000 & 4.71710900 & -1.36397800 & $\mathrm{C}$ & -0.09657000 & -5.15540700 & -0.86945200 \\
\hline $\mathrm{C}$ & 5.24723200 & 4.43623900 & -2.21344400 & $\mathrm{H}$ & -0.27835300 & -5.86061100 & -0.06062300 \\
\hline $\mathrm{H}$ & 5.99029200 & 3.85595000 & -1.66306200 & $\mathrm{H}$ & 0.97904000 & -5.01187200 & -0.96767700 \\
\hline $\mathrm{H}$ & 5.71415100 & 5.37991600 & -2.50309900 & $\mathrm{H}$ & -0.44415300 & -5.62376100 & -1.79502100 \\
\hline $\mathrm{H}$ & 5.00123800 & 3.89501100 & -3.12902600 & $\mathrm{C}$ & -0.48002100 & -2.90679300 & -1.87521700 \\
\hline $\mathrm{C}$ & 3.03722300 & 5.58227500 & -2.20580100 & $\mathrm{H}$ & -0.74426800 & -3.42360000 & -2.80247100 \\
\hline $\mathrm{H}$ & 2.71943200 & 5.04709600 & -3.10296300 & $\mathrm{H}$ & 0.58377000 & -2.67717300 & -1.91468800 \\
\hline $\mathrm{H}$ & 3.52878500 & 6.50735900 & -2.51834700 & $\mathrm{H}$ & -1.03592300 & -1.97106300 & -1.84587600 \\
\hline $\mathrm{H}$ & 2.14179200 & 5.85425400 & -1.64538200 & $\mathrm{C}$ & 1.01626800 & 1.61553600 & 3.78946200 \\
\hline $\mathrm{C}$ & 4.44128700 & 5.51705200 & -0.12077900 & $\mathrm{C}$ & 2.17363000 & 0.68895900 & 3.37236400 \\
\hline $\mathrm{H}$ & 3.59290100 & 5.79371500 & 0.50656100 & $\mathrm{H}$ & 2.94219500 & 0.72242400 & 4.15033300 \\
\hline $\mathrm{H}$ & 4.94787800 & 6.43855200 & -0.41950300 & $\mathrm{H}$ & 2.63245300 & 0.99247200 & 2.43481100 \\
\hline $\mathrm{H}$ & 5.13183600 & 4.93268400 & 0.49069900 & $\mathrm{H}$ & 1.84652500 & -0.34487000 & 3.26657900 \\
\hline $\mathrm{C}$ & 5.50502000 & -3.31106500 & -2.08930200 & $\mathrm{C}$ & 0.50446700 & 1.11679600 & 5.15100900 \\
\hline $\mathrm{C}$ & 5.39536600 & -4.82006500 & -1.82287100 & $\mathrm{H}$ & 0.08136500 & 0.11279900 & 5.08474300 \\
\hline $\mathrm{H}$ & 6.20256300 & -5.34268500 & -2.34024300 & $\mathrm{H}$ & -0.24171200 & 1.77425500 & 5.59326800 \\
\hline $\mathrm{H}$ & 4.45115600 & -5.22963700 & -2.18744800 & $\mathrm{H}$ & 1.34768000 & 1.06936800 & 5.84579800 \\
\hline $\mathrm{H}$ & 5.48212700 & -5.05368000 & -0.75982100 & $\mathrm{C}$ & 1.57532300 & 3.03999800 & 3.93385900 \\
\hline $\mathrm{C}$ & 6.87926500 & -2.83746500 & -1.57132300 & $\mathrm{H}$ & 2.36192100 & 3.03577800 & 4.69475100 \\
\hline $\mathrm{H}$ & 6.96000900 & -2.98638200 & -0.49259400 & $\mathrm{H}$ & 0.82432900 & 3.76266600 & 4.24661700 \\
\hline $\mathrm{H}$ & 7.04345100 & -1.77848600 & -1.77353900 & $\mathrm{H}$ & 2.02669000 & 3.39007800 & 3.00579800 \\
\hline $\mathrm{H}$ & 7.68441800 & -3.39809400 & -2.05370200 & $\mathrm{C}$ & -1.99012000 & 2.24659600 & 3.10334400 \\
\hline $\mathrm{C}$ & 5.43854000 & -3.09402900 & -3.61561100 & $\mathrm{C}$ & -2.70546000 & 1.19788500 & 3.97533400 \\
\hline $\mathrm{H}$ & 6.23336900 & -3.65250800 & -4.11725800 & $\mathrm{H}$ & -2.14701200 & 0.93616400 & 4.87178400 \\
\hline $\mathrm{H}$ & 5.55310200 & -2.04169100 & -3.87795600 & $\mathrm{H}$ & -2.89286700 & 0.28224200 & 3.41272800 \\
\hline $\mathrm{H}$ & 4.48053300 & -3.43328800 & -4.01494800 & $\mathrm{H}$ & -3.67307400 & 1.59562800 & 4.29455300 \\
\hline $\mathrm{C}$ & -1.02167800 & -3.73327300 & 2.46835800 & $\mathrm{C}$ & -1.82631400 & 3.55548400 & 3.88999800 \\
\hline $\mathrm{C}$ & -2.50447600 & -3.34929800 & 2.62476900 & $\mathrm{H}$ & -2.81825100 & 3.94981300 & 4.13080900 \\
\hline $\mathrm{H}$ & -2.87810600 & -3.74196300 & 3.57488100 & $\mathrm{H}$ & -1.30892000 & 4.32067700 & 3.30960800 \\
\hline $\mathrm{H}$ & -3.13063100 & -3.75381000 & 1.83281300 & $\mathrm{H}$ & -1.29569400 & 3.42242800 & 4.83098700 \\
\hline $\mathrm{H}$ & -2.63420200 & -2.26633200 & 2.63483900 & $\mathrm{C}$ & -2.90199500 & 2.53399300 & 1.89552300 \\
\hline $\mathrm{C}$ & -0.26530700 & -3.14253900 & 3.67347200 & $\mathrm{H}$ & -3.01518900 & 1.66545900 & 1.24962600 \\
\hline
\end{tabular}




\begin{tabular}{|c|c|c|c|c|c|c|c|}
\hline $\mathrm{H}$ & -2.54572600 & 3.36747100 & 1.29143100 & $\mathrm{C}$ & -3.06626200 & 3.25997500 & -0.19854000 \\
\hline $\mathrm{H}$ & -3.89370000 & 2.80488000 & 2.26826700 & $\mathrm{C}$ & -3.40197200 & 1.93725800 & -0.54911800 \\
\hline $\mathrm{C}$ & -3.94184500 & 0.43821500 & -1.56601400 & $\mathrm{H}$ & -4.44119500 & 1.69541400 & -0.74060100 \\
\hline $\mathrm{C}$ & -3.50040400 & 1.76329500 & -2.04933100 & $\mathrm{C}$ & -2.47346300 & 0.91562500 & -0.70155500 \\
\hline $\mathrm{C}$ & -2.14390300 & 2.03984100 & -2.27352200 & $\mathrm{C}$ & -1.11664900 & 1.20766200 & -0.48063300 \\
\hline $\mathrm{H}$ & -1.40685600 & 1.26397900 & -2.11934400 & $\mathrm{C}$ & -2.90186000 & -0.43556700 & -1.18854300 \\
\hline $\mathrm{C}$ & -1.72984100 & 3.29298000 & -2.70554600 & $\mathrm{H}$ & -2.45329400 & -0.61137100 & -2.16869600 \\
\hline $\mathrm{H}$ & -0.67621500 & 3.47470500 & -2.87742900 & $\mathrm{H}$ & -3.98378900 & -0.47823900 & -1.31086800 \\
\hline $\mathrm{C}$ & -2.65834600 & 4.30604900 & -2.91923100 & $\mathrm{C}$ & 2.92748000 & -0.32314900 & -1.19731700 \\
\hline $\mathrm{H}$ & -2.33478200 & 5.28324800 & -3.25468700 & $\mathrm{H}$ & 4.00996500 & -0.32324300 & -1.32216600 \\
\hline $\mathrm{C}$ & -4.00764300 & 4.04972000 & -2.69631600 & $\mathrm{H}$ & 2.48409600 & -0.51926100 & -2.17604200 \\
\hline $\mathrm{H}$ & -4.74114600 & 4.83182600 & -2.85042000 & $\mathrm{C}$ & 3.96520800 & 4.52162200 & -0.04772600 \\
\hline $\mathrm{C}$ & -4.42422900 & 2.79697200 & -2.26881900 & $\mathrm{C}$ & 3.86248900 & 5.10221100 & 1.37813500 \\
\hline $\mathrm{H}$ & -5.47598200 & 2.61754200 & -2.09044400 & $\mathrm{H}$ & 2.86136700 & 5.48092400 & 1.58714500 \\
\hline $\mathrm{C}$ & -5.22950000 & -0.16709600 & -1.95114300 & $\mathrm{H}$ & 4.56679400 & 5.92841100 & 1.50766300 \\
\hline $\mathrm{C}$ & -5.89064600 & -1.05716900 & -1.09034000 & $\mathrm{H}$ & 4.09177500 & 4.33974300 & 2.12548800 \\
\hline $\mathrm{H}$ & -5.46021300 & -1.26293300 & -0.11887700 & $\mathrm{C}$ & 5.41635200 & 4.06862700 & -0.26918300 \\
\hline $\mathrm{C}$ & -7.08152900 & -1.66340300 & -1.46064200 & $\mathrm{H}$ & 5.71301200 & 3.29389700 & 0.44082400 \\
\hline $\mathrm{H}$ & -7.57198100 & -2.34232600 & -0.77346000 & $\mathrm{H}$ & 6.09022800 & 4.91686100 & -0.13161600 \\
\hline $\mathrm{C}$ & -7.65438300 & -1.39399300 & -2.70021500 & $\mathrm{H}$ & 5.57356600 & 3.68513800 & -1.27929300 \\
\hline $\mathrm{H}$ & -8.58682300 & -1.86321100 & -2.98722200 & $\mathrm{C}$ & 3.65534100 & 5.63564000 & -1.06960500 \\
\hline $\mathrm{C}$ & -7.01396200 & -0.51277400 & -3.56413800 & $\mathrm{H}$ & 3.72880600 & 5.25667700 & -2.09100600 \\
\hline $\mathrm{H}$ & -7.44157500 & -0.30012800 & -4.53641600 & $\mathrm{H}$ & 4.36207100 & 6.46285800 & -0.96139500 \\
\hline $\mathrm{C}$ & -5.81915200 & 0.09218700 & -3.19702000 & $\mathrm{H}$ & 2.64989600 & 6.03628200 & -0.93596300 \\
\hline $\mathrm{H}$ & -5.32661500 & 0.75907200 & -3.89185700 & $\mathrm{C}$ & -4.17598500 & 4.31248800 & -0.05143300 \\
\hline & & & & $\mathrm{C}$ & -5.16993100 & 3.87363300 & 1.04421900 \\
\hline \multirow{2}{*}{\multicolumn{4}{|c|}{$\left[{ }^{t \mathrm{Bu}}(\mathrm{PNP}) \mathrm{Fe}(\mathrm{CO})\right](6)$, et }} & $\mathrm{H}$ & -5.63744000 & 2.91714500 & 0.80625900 \\
\hline & & & & $\mathrm{H}$ & -5.96635800 & 4.61390900 & 1.15787000 \\
\hline $\mathrm{Fe}$ & 0.03792600 & -1.57439900 & 0.06385700 & $\mathrm{H}$ & -4.66473300 & 3.76762700 & 2.00640800 \\
\hline $\mathrm{P}$ & 2.35279100 & -1.72120600 & -0.13490700 & $\mathrm{C}$ & -4.93281700 & 4.46639700 & -1.38752100 \\
\hline $\mathrm{P}$ & -2.26878700 & -1.81184800 & -0.13065900 & $\mathrm{H}$ & -4.25582500 & 4.78802300 & -2.18143100 \\
\hline $\mathrm{O}$ & 0.08394200 & -3.74128300 & 1.98828800 & $\mathrm{H}$ & -5.72697800 & 5.21198700 & -1.29419900 \\
\hline $\mathrm{N}$ & -0.00060100 & 0.37242600 & -0.58705400 & $\mathrm{H}$ & -5.39291300 & 3.52881500 & -1.70250600 \\
\hline $\mathrm{C}$ & 1.08420000 & 1.24870600 & -0.48586100 & $\mathrm{C}$ & -3.62098200 & 5.69036500 & 0.33924000 \\
\hline $\mathrm{C}$ & 2.44726900 & 1.01057300 & -0.71040000 & $\mathrm{H}$ & -3.08910800 & 5.65717100 & 1.29206200 \\
\hline $\mathrm{C}$ & 3.34185800 & 2.06854800 & -0.56111400 & $\mathrm{H}$ & -4.44295700 & 6.40176600 & 0.44341200 \\
\hline $\mathrm{H}$ & 4.38552400 & 1.86092200 & -0.75575600 & $\mathrm{H}$ & -2.93972100 & 6.08277600 & -0.41807000 \\
\hline $\mathrm{C}$ & 2.95587700 & 3.37267600 & -0.21045900 & $\mathrm{C}$ & -2.79034400 & -3.35404800 & -1.14529800 \\
\hline $\mathrm{C}$ & 1.59462500 & 3.60870300 & -0.04183400 & $\mathrm{C}$ & -2.16304900 & -3.20614300 & -2.54666100 \\
\hline $\mathrm{H}$ & 1.23451200 & 4.60447700 & 0.18797600 & $\mathrm{H}$ & -2.64385500 & -2.43202700 & -3.14316200 \\
\hline $\mathrm{C}$ & 0.67445700 & 2.57569800 & -0.19372900 & $\mathrm{H}$ & -2.27610800 & -4.15038100 & -3.08607400 \\
\hline C & -0.75998900 & 2.54679300 & -0.19092800 & $\mathrm{H}$ & -1.09580500 & -2.98285400 & -2.49597100 \\
\hline $\mathrm{C}$ & -1.71882800 & 3.54918900 & -0.03444200 & $\mathrm{C}$ & -2.22749800 & -4.64761200 & -0.53197900 \\
\hline $\mathrm{H}$ & -1.39150400 & 4.55389800 & 0.19515500 & $\mathrm{H}$ & -1.13950600 & -4.63549300 & -0.49853100 \\
\hline
\end{tabular}




\begin{tabular}{|c|c|c|c|c|c|c|c|}
\hline $\mathrm{H}$ & -2.53060600 & -5.49228400 & -1.15755400 & $\mathrm{H}$ & 1.59311700 & -0.98835700 & 2.65284200 \\
\hline $\mathrm{H}$ & -2.58992800 & -4.83853900 & 0.47469600 & $\mathrm{C}$ & 0.06630300 & -2.92412100 & 1.15994200 \\
\hline $\mathrm{C}$ & -4.31174200 & -3.49652400 & -1.30906600 & & & & \\
\hline $\mathrm{H}$ & -4.80938000 & -3.72801000 & -0.36837300 & \multirow{2}{*}{\multicolumn{4}{|c|}{$\left[{ }^{t \mathrm{Bu}}(\mathrm{PNP}) \mathrm{Fe}(\mathrm{CO})\right](6), 4 \mathrm{et}$}} \\
\hline $\mathrm{H}$ & -4.52055700 & -4.32152400 & -1.99685900 & & & & \\
\hline $\mathrm{H}$ & -4.77131300 & -2.60142300 & -1.72920900 & $\mathrm{Fe}$ & -0.00694900 & -1.37658800 & 0.46805600 \\
\hline $\mathrm{C}$ & -3.28411300 & -1.68101600 & 1.49368600 & $\mathrm{P}$ & 2.43013000 & -1.89069500 & -0.11643600 \\
\hline $\mathrm{C}$ & -4.70982700 & -1.14907000 & 1.27215000 & $\mathrm{P}$ & -2.44527000 & -1.86142500 & -0.13579800 \\
\hline $\mathrm{H}$ & -4.70939900 & -0.13473700 & 0.87764700 & $\mathrm{O}$ & -0.01465800 & -1.94146700 & 3.41200400 \\
\hline $\mathrm{H}$ & -5.22469600 & -1.12060700 & 2.23695000 & $\mathrm{~N}$ & 0.00605500 & 0.39246200 & -0.58488600 \\
\hline $\mathrm{H}$ & -5.29981800 & -1.78013500 & 0.60893600 & $\mathrm{C}$ & 1.11336800 & 1.22474900 & -0.49317000 \\
\hline $\mathrm{C}$ & -2.53009200 & -0.68528500 & 2.39847100 & $\mathrm{C}$ & 2.45800400 & 0.90383800 & -0.72631700 \\
\hline $\mathrm{H}$ & -1.53599900 & -1.05030500 & 2.65651400 & $\mathrm{C}$ & 3.39780200 & 1.92552300 & -0.60454800 \\
\hline $\mathrm{H}$ & -3.09210500 & -0.56029700 & 3.32882800 & $\mathrm{H}$ & 4.43105000 & 1.67528300 & -0.80439800 \\
\hline $\mathrm{H}$ & -2.42556400 & 0.29542400 & 1.93698400 & $\mathrm{C}$ & 3.06607500 & 3.25205300 & -0.27431100 \\
\hline $\mathrm{C}$ & -3.36994500 & -3.02515100 & 2.23585600 & $\mathrm{C}$ & 1.71760200 & 3.56008400 & -0.10077700 \\
\hline $\mathrm{H}$ & -4.00355700 & -3.74575200 & 1.71963300 & $\mathrm{H}$ & 1.41286000 & 4.57805700 & 0.11103800 \\
\hline $\mathrm{H}$ & -3.82056800 & -2.84677300 & 3.21653100 & $\mathrm{C}$ & 0.74665500 & 2.57025800 & -0.22480700 \\
\hline $\mathrm{H}$ & -2.39396600 & -3.47660200 & 2.40467400 & $\mathrm{C}$ & -0.69481800 & 2.58110400 & -0.21515000 \\
\hline $\mathrm{C}$ & 2.93333400 & -3.24437000 & -1.14668000 & $\mathrm{C}$ & -1.64783700 & 3.59217900 & -0.07569900 \\
\hline $\mathrm{C}$ & 4.45872300 & -3.32429500 & -1.31547200 & $\mathrm{H}$ & -1.31862100 & 4.60017600 & 0.13584600 \\
\hline $\mathrm{H}$ & 4.87948000 & -2.41206500 & -1.73937400 & $\mathrm{C}$ & -2.99888000 & 3.30549700 & -0.23255000 \\
\hline $\mathrm{H}$ & 4.69921200 & -4.14181800 & -2.00185600 & $\mathrm{C}$ & -3.35529600 & 1.98109900 & -0.56527600 \\
\hline $\mathrm{H}$ & 4.96853500 & -3.53254400 & -0.37587900 & $\mathrm{H}$ & -4.39819700 & 1.75502300 & -0.75630000 \\
\hline $\mathrm{C}$ & 2.42659400 & -4.55862300 & -0.52837900 & $\mathrm{C}$ & -2.43950900 & 0.94598200 & -0.70192000 \\
\hline $\mathrm{H}$ & 2.80130100 & -4.73278800 & 0.47685600 & $\mathrm{C}$ & -1.08433000 & 1.24507400 & -0.47950700 \\
\hline $\mathrm{H}$ & 2.76111800 & -5.39135400 & -1.15398300 & $\mathrm{C}$ & -2.86220100 & -0.40563000 & -1.22019500 \\
\hline $\mathrm{H}$ & 1.33917900 & -4.59115800 & -0.48976900 & $\mathrm{H}$ & -2.30984500 & -0.59377500 & -2.14263100 \\
\hline $\mathrm{C}$ & 2.29558300 & -3.12625200 & -2.54616000 & $\mathrm{H}$ & -3.92330500 & -0.40050300 & -1.47202600 \\
\hline $\mathrm{H}$ & 1.22001400 & -2.94841600 & -2.49196100 & $\mathrm{C}$ & 2.85682200 & -0.46024100 & -1.23078800 \\
\hline $\mathrm{H}$ & 2.44668500 & -4.06608600 & -3.08397200 & $\mathrm{H}$ & 3.91568600 & -0.47364900 & -1.49165600 \\
\hline $\mathrm{H}$ & 2.74096200 & -2.33374500 & -3.14596300 & $\mathrm{H}$ & 2.29333200 & -0.65692700 & -2.14488200 \\
\hline C & 3.36437500 & -1.54627900 & 1.48778600 & $\mathrm{C}$ & 4.12731400 & 4.35847300 & -0.14054100 \\
\hline $\mathrm{C}$ & 3.50827400 & -2.88502400 & 2.23053600 & $\mathrm{C}$ & 4.05928100 & 4.97236300 & 1.27345700 \\
\hline $\mathrm{H}$ & 2.55262800 & -3.37719200 & 2.40188400 & $\mathrm{H}$ & 3.07759700 & 5.39997800 & 1.48005300 \\
\hline $\mathrm{H}$ & 3.95332800 & -2.68724700 & 3.21003800 & $\mathrm{H}$ & 4.80073200 & 5.76857300 & 1.38193600 \\
\hline $\mathrm{H}$ & 4.17046900 & -3.57842100 & 1.71300800 & $\mathrm{H}$ & 4.25865200 & 4.21601100 & 2.03540700 \\
\hline $\mathrm{C}$ & 4.76606900 & -0.95513800 & 1.26310900 & $\mathrm{C}$ & 5.55604000 & 3.83748200 & -0.35891400 \\
\hline $\mathrm{H}$ & 5.38064900 & -1.56127500 & 0.59896100 & $\mathrm{H}$ & 5.82130000 & 3.06366200 & 0.36435500 \\
\hline $\mathrm{H}$ & 5.28123900 & -0.90498600 & 2.22691900 & $\mathrm{H}$ & 6.26712300 & 4.65763700 & -0.23958100 \\
\hline $\mathrm{H}$ & 4.72266100 & 0.05804300 & 0.86797700 & $\mathrm{H}$ & 5.69201700 & 3.42914800 & -1.36237700 \\
\hline $\mathrm{C}$ & 2.57084800 & -0.58244300 & 2.39321600 & $\mathrm{C}$ & 3.86255200 & 5.46408000 & -1.18408000 \\
\hline $\mathrm{H}$ & 2.42505600 & 0.39271100 & 1.93115400 & $\mathrm{H}$ & 3.91202100 & 5.06087000 & -2.19763000 \\
\hline $\mathrm{H}$ & 3.12837900 & -0.43383100 & 3.32282700 & $\mathrm{H}$ & 4.60732700 & 6.25985600 & -1.09716800 \\
\hline
\end{tabular}




\begin{tabular}{|c|c|c|c|c|c|c|c|}
\hline $\mathrm{H}$ & 2.87758300 & 5.91363000 & -1.05373000 & $\mathrm{H}$ & 4.81745100 & -4.02126900 & -0.60640700 \\
\hline $\mathrm{C}$ & -4.09938700 & 4.37075300 & -0.09850400 & $\mathrm{C}$ & 2.16089500 & -4.65750400 & -0.51343800 \\
\hline $\mathrm{C}$ & -5.09395000 & 3.95677400 & 1.00633400 & $\mathrm{H}$ & 2.62825100 & -4.89061200 & 0.44107900 \\
\hline $\mathrm{H}$ & -5.56974700 & 3.00053600 & 0.78444700 & $\mathrm{H}$ & 2.27515300 & -5.53237600 & -1.16017300 \\
\hline $\mathrm{H}$ & -5.88394500 & 4.70528300 & 1.11087400 & $\mathrm{H}$ & 1.09492000 & -4.51327000 & -0.33546400 \\
\hline $\mathrm{H}$ & -4.58690700 & 3.86123700 & 1.96861800 & $\mathrm{C}$ & 2.06759700 & -3.22202600 & -2.54433300 \\
\hline $\mathrm{C}$ & -4.85872200 & 4.51271200 & -1.43456600 & $\mathrm{H}$ & 1.04046200 & -2.88163600 & -2.41306100 \\
\hline $\mathrm{H}$ & -4.18095000 & 4.81621000 & -2.23494100 & $\mathrm{H}$ & 2.03617500 & -4.17332700 & -3.08241500 \\
\hline $\mathrm{H}$ & -5.64516400 & 5.26745700 & -1.34971200 & $\mathrm{H}$ & 2.58338900 & -2.50372600 & -3.18083300 \\
\hline $\mathrm{H}$ & -5.32931300 & 3.57555600 & -1.73493200 & $\mathrm{C}$ & 3.70101200 & -1.76463800 & 1.31437800 \\
\hline $\mathrm{C}$ & -3.53266800 & 5.74983300 & 0.27080400 & $\mathrm{C}$ & 3.70225700 & -3.06408300 & 2.13525200 \\
\hline $\mathrm{H}$ & -2.99803700 & 5.72627400 & 1.22232000 & $\mathrm{H}$ & 2.69584600 & -3.35822400 & 2.43689100 \\
\hline $\mathrm{H}$ & -4.34923400 & 6.46848900 & 0.36751900 & $\mathrm{H}$ & 4.28273500 & -2.90544000 & 3.04842500 \\
\hline $\mathrm{H}$ & -2.85120900 & 6.12678000 & -0.49415100 & $\mathrm{H}$ & 4.16182900 & -3.89570000 & 1.60132100 \\
\hline $\mathrm{C}$ & -2.80184400 & -3.37445300 & -1.25698400 & $\mathrm{C}$ & 5.13397000 & -1.44459500 & 0.85618800 \\
\hline $\mathrm{C}$ & -2.08263800 & -3.13214700 & -2.59733700 & $\mathrm{H}$ & 5.55619500 & -2.19830000 & 0.19644100 \\
\hline $\mathrm{H}$ & -2.60845300 & -2.41015500 & -3.22140900 & $\mathrm{H}$ & 5.77669300 & -1.38774400 & 1.73973400 \\
\hline $\mathrm{H}$ & -2.03754200 & -4.07313200 & -3.15225800 & $\mathrm{H}$ & 5.19358900 & -0.47792400 & 0.35734900 \\
\hline $\mathrm{H}$ & -1.06067200 & -2.77867100 & -2.45981000 & $\mathrm{C}$ & 3.24763000 & -0.61701100 & 2.23998800 \\
\hline $\mathrm{C}$ & -2.17135200 & -4.61538500 & -0.60005700 & $\mathrm{H}$ & 3.19102500 & 0.33716700 & 1.71975500 \\
\hline $\mathrm{H}$ & -1.10371500 & -4.47601300 & -0.42858000 & $\mathrm{H}$ & 3.98147700 & -0.51551300 & 3.04525000 \\
\hline $\mathrm{H}$ & -2.29231300 & -5.47748400 & -1.26251200 & $\mathrm{H}$ & 2.28327500 & -0.81460400 & 2.69939400 \\
\hline $\mathrm{H}$ & -2.63131300 & -4.86595800 & 0.35378000 & $\mathrm{C}$ & -0.01281600 & -1.70301500 & 2.28223500 \\
\hline $\mathrm{C}$ & -4.27991900 & -3.65792900 & -1.55866900 & & & & \\
\hline $\mathrm{H}$ & -4.82860000 & -3.98601100 & -0.67729700 & \multirow{2}{*}{\multicolumn{4}{|c|}{$\left[\left({ }^{t \mathrm{Bu}}(\mathrm{PNP}) \mathrm{Fe}\right)_{2}\left(\mathrm{CO}_{3}\right)\right](7)$, et }} \\
\hline $\mathrm{H}$ & -4.34392300 & -4.46364300 & -2.29675700 & & & & \\
\hline $\mathrm{H}$ & -4.78966000 & -2.79087000 & -1.98049700 & $\mathrm{Fe}$ & -2.24352916 & -1.11417408 & 1.12516608 \\
\hline $\mathrm{C}$ & -3.72383800 & -1.76319100 & 1.28977600 & $\mathrm{Fe}$ & 2.46146418 & -0.95501507 & -1.66968012 \\
\hline $\mathrm{C}$ & -5.15592300 & -1.43829700 & 0.83230500 & $\mathrm{P}$ & -1.69692012 & 0.49018104 & 3.07349822 \\
\hline $\mathrm{H}$ & -5.21549900 & -0.46444400 & 0.34741000 & $\mathrm{P}$ & -3.77729427 & -3.13424623 & 1.13930308 \\
\hline $\mathrm{H}$ & -5.80100900 & -1.39469500 & 1.71482700 & $\mathrm{P}$ & 1.87761514 & 0.87488806 & -3.24026023 \\
\hline $\mathrm{H}$ & -5.57563600 & -2.18264200 & 0.16057700 & $\mathrm{P}$ & 4.13995730 & -2.80363320 & -1.56539411 \\
\hline $\mathrm{C}$ & -3.27669600 & -0.63007000 & 2.23595700 & $\mathrm{O}$ & -0.67749005 & -2.30989117 & 0.60962204 \\
\hline $\mathrm{H}$ & -2.31281600 & -0.83194000 & 2.69433700 & $\mathrm{O}$ & 0.99200907 & -1.87045013 & -0.81146306 \\
\hline $\mathrm{H}$ & -4.01290600 & -0.54405900 & 3.04080100 & $\mathrm{O}$ & -0.49513704 & -0.28109202 & -0.22191502 \\
\hline $\mathrm{H}$ & -3.22198700 & 0.33300400 & 1.73191900 & $\mathrm{~N}$ & -3.71689227 & 0.16373601 & 0.62199904 \\
\hline $\mathrm{C}$ & -3.72462800 & -3.07703700 & 2.08727700 & $\mathrm{~N}$ & 3.32253524 & 0.19885801 & -0.28517002 \\
\hline $\mathrm{H}$ & -4.17710600 & -3.90084700 & 1.53536600 & $\mathrm{C}$ & -4.91734335 & -0.12144101 & -0.01609600 \\
\hline $\mathrm{H}$ & -4.31108800 & -2.93772500 & 2.99977600 & $\mathrm{C}$ & -5.71900342 & -1.28204809 & 0.06379200 \\
\hline $\mathrm{H}$ & -2.71858800 & -3.37218900 & 2.38922700 & $\mathrm{C}$ & -6.87805152 & -1.31187910 & -0.70835005 \\
\hline $\mathrm{C}$ & 2.78832700 & -3.43070200 & -1.19929500 & $\mathrm{H}$ & -7.49936355 & -2.19915316 & -0.63779904 \\
\hline $\mathrm{C}$ & 4.26698500 & -3.71735400 & -1.49524200 & $\mathrm{C}$ & -7.31359852 & -0.24374402 & -1.52615211 \\
\hline $\mathrm{H}$ & 4.77374000 & -2.85968100 & -1.93932500 & $\mathrm{C}$ & -6.55942347 & 0.92559807 & -1.51472611 \\
\hline $\mathrm{H}$ & 4.33285100 & -4.54126800 & -2.21279000 & $\mathrm{H}$ & -6.87431648 & 1.79425113 & -2.07916715 \\
\hline
\end{tabular}




\begin{tabular}{|c|c|c|c|c|c|c|c|}
\hline $\mathrm{C}$ & -5.38600239 & 0.99698007 & -0.75571706 & $\mathrm{H}$ & 0.96017907 & -1.22358209 & 5.18164437 \\
\hline $\mathrm{C}$ & -4.46317632 & 2.06832715 & -0.47252303 & $\mathrm{H}$ & -0.35449103 & -1.87987014 & 4.20409230 \\
\hline $\mathrm{C}$ & -4.44650932 & 3.42230325 & -0.82237806 & $\mathrm{C}$ & 0.46259603 & 1.45847611 & 4.78489734 \\
\hline $\mathrm{H}$ & -5.21523038 & 3.80628527 & -1.48106611 & $\mathrm{H}$ & 0.50642204 & 2.38255017 & 4.20397730 \\
\hline $\mathrm{C}$ & -3.46840825 & 4.26150831 & -0.29846102 & $\mathrm{H}$ & 1.47451311 & 1.26520709 & 5.15802337 \\
\hline $\mathrm{C}$ & -2.52010018 & 3.69596727 & 0.58455604 & $\mathrm{H}$ & -0.17644601 & 1.62656111 & 5.65282039 \\
\hline $\mathrm{H}$ & -1.76180213 & 4.34209231 & 1.01506407 & $\mathrm{C}$ & 1.06025007 & 0.01450000 & 2.83251620 \\
\hline $\mathrm{C}$ & -2.48630918 & 2.34993017 & 0.94836807 & $\mathrm{H}$ & 0.80070606 & -0.84574506 & 2.21560216 \\
\hline $\mathrm{C}$ & -3.47689525 & 1.51483111 & 0.38726603 & $\mathrm{H}$ & 2.01908614 & -0.20250801 & 3.31415424 \\
\hline $\mathrm{C}$ & -1.36419410 & 1.88214114 & 1.86001614 & $\mathrm{H}$ & 1.21528609 & 0.87550906 & 2.18344716 \\
\hline $\mathrm{H}$ & -0.95007107 & 2.74384320 & 2.38811117 & $\mathrm{C}$ & -2.98139622 & 1.21165909 & 4.31634231 \\
\hline $\mathrm{H}$ & -0.57253104 & 1.47239411 & 1.22883009 & $\mathrm{C}$ & -2.94216621 & 0.45126403 & 5.65472739 \\
\hline $\mathrm{C}$ & -5.48014938 & -2.37801117 & 1.08329908 & $\mathrm{H}$ & -2.03268015 & 0.64429604 & 6.22425144 \\
\hline $\mathrm{H}$ & -6.24479743 & -3.15116022 & 0.97968207 & $\mathrm{H}$ & -3.04076122 & -0.62829405 & 5.51662340 \\
\hline $\mathrm{H}$ & -5.61112741 & -1.93610814 & 2.07523315 & $\mathrm{H}$ & -3.78665227 & 0.78069606 & 6.26977645 \\
\hline $\mathrm{C}$ & -8.60954064 & -0.39792403 & -2.34396917 & $\mathrm{C}$ & -2.78323020 & 2.71746619 & 4.57297833 \\
\hline $\mathrm{C}$ & -8.92252464 & 0.85640006 & -3.18035123 & $\mathrm{H}$ & -3.54799725 & 3.06024522 & 5.27953638 \\
\hline $\mathrm{H}$ & -9.07672663 & 1.73635413 & -2.55015519 & $\mathrm{H}$ & -2.90293421 & 3.29978624 & 3.65807226 \\
\hline $\mathrm{H}$ & -9.83949071 & 0.69704505 & -3.75486427 & $\mathrm{H}$ & -1.81022413 & 2.95026021 & 5.00618736 \\
\hline $\mathrm{H}$ & -8.12070956 & 1.07999508 & -3.88930328 & $\mathrm{C}$ & -4.38465031 & 1.00759507 & 3.70911526 \\
\hline $\mathrm{C}$ & -9.80494268 & -0.64678805 & -1.39431010 & $\mathrm{H}$ & -4.58873133 & -0.04516100 & 3.50861125 \\
\hline $\mathrm{H}$ & -9.67040868 & -1.55496811 & -0.80206006 & $\mathrm{H}$ & -4.51771533 & 1.55576411 & 2.77839320 \\
\hline $\mathrm{H}$ & -10.73300575 & -0.75578706 & -1.96532014 & $\mathrm{H}$ & -5.13108437 & 1.36870210 & 4.42589632 \\
\hline $\mathrm{H}$ & -9.92771773 & 0.18861401 & -0.69969605 & $\mathrm{C}$ & -3.82486327 & -4.26487031 & 2.68515319 \\
\hline $\mathrm{C}$ & -8.47425859 & -1.59429011 & -3.31457324 & $\mathrm{C}$ & -4.26957731 & -3.37648924 & 3.86665928 \\
\hline $\mathrm{H}$ & -7.64183553 & -1.43941210 & -4.00655829 & $\mathrm{H}$ & -3.68936126 & -2.45129618 & 3.91531028 \\
\hline $\mathrm{H}$ & -9.38946567 & -1.71705212 & -3.90322828 & $\mathrm{H}$ & -4.10756930 & -3.92127728 & 4.80260035 \\
\hline $\mathrm{H}$ & -8.29340661 & -2.53012818 & -2.78062020 & $\mathrm{H}$ & -5.32911939 & -3.11797622 & 3.81962328 \\
\hline $\mathrm{C}$ & -3.39354924 & 5.76449341 & -0.62722705 & $\mathrm{C}$ & -2.38654317 & -4.74178534 & 2.97782122 \\
\hline $\mathrm{C}$ & -2.04414115 & 6.07974546 & -1.31395509 & $\mathrm{H}$ & -2.01336515 & -5.43479741 & 2.22529416 \\
\hline $\mathrm{H}$ & -1.19760609 & 5.81693842 & -0.67555805 & $\mathrm{H}$ & -2.36973917 & -5.25907138 & 3.94359729 \\
\hline $\mathrm{H}$ & -1.96993714 & 7.14715450 & -1.54742111 & $\mathrm{H}$ & -1.68779212 & -3.90399528 & 3.02812822 \\
\hline $\mathrm{H}$ & -1.94620714 & 5.51868041 & -2.24771816 & $\mathrm{C}$ & -4.76984735 & -5.47322737 & 2.58805419 \\
\hline $\mathrm{C}$ & -3.50845925 & 6.59536746 & 0.67205005 & $\mathrm{H}$ & -5.79457443 & -5.17790137 & 2.34861717 \\
\hline $\mathrm{H}$ & -4.45756832 & 6.39466244 & 1.17651208 & $\mathrm{H}$ & -4.79709034 & -5.99175641 & 3.55350625 \\
\hline $\mathrm{H}$ & -3.46042625 & 7.66622256 & 0.44818803 & $\mathrm{H}$ & -4.43755832 & -6.19680545 & 1.84213813 \\
\hline $\mathrm{H}$ & -2.70310120 & 6.36614645 & 1.37339710 & $\mathrm{C}$ & -3.65829326 & -4.15165630 & -0.48009503 \\
\hline $\mathrm{C}$ & -4.52206833 & 6.21105344 & -1.57484611 & $\mathrm{C}$ & -2.49040218 & -5.15130237 & -0.39667403 \\
\hline $\mathrm{H}$ & -4.48118132 & 5.68284141 & -2.53131018 & $\mathrm{H}$ & -1.57021011 & -4.65861634 & -0.07522001 \\
\hline $\mathrm{H}$ & -4.42718532 & 7.28067253 & -1.78284813 & $\mathrm{H}$ & -2.31756217 & -5.57386439 & -1.39297510 \\
\hline $\mathrm{H}$ & -5.50926540 & 6.04529544 & -1.13517708 & $\mathrm{H}$ & -2.70548319 & -5.98602944 & 0.27353302 \\
\hline $\mathrm{C}$ & 0.00724000 & 0.26218002 & 3.93219428 & $\mathrm{C}$ & -3.33484924 & -3.13914623 & -1.60203411 \\
\hline $\mathrm{C}$ & -0.04409300 & -1.01578107 & 4.79679035 & $\mathrm{H}$ & -4.08957430 & -2.35647917 & -1.68727412 \\
\hline $\mathrm{H}$ & -0.71159605 & -0.93125007 & 5.65302439 & $\mathrm{H}$ & -3.29890524 & -3.67434127 & -2.55780518 \\
\hline
\end{tabular}




\begin{tabular}{|c|c|c|c|c|c|c|c|}
\hline $\mathrm{H}$ & -2.35927217 & -2.67616019 & -1.44783310 & $\mathrm{H}$ & 1.57174411 & 5.74905942 & 2.19738716 \\
\hline $\mathrm{C}$ & -4.96030236 & -4.88494035 & -0.84842606 & $\mathrm{C}$ & 3.58474626 & 6.62678445 & -0.50501304 \\
\hline $\mathrm{H}$ & -5.27504638 & -5.60160842 & -0.08937801 & $\mathrm{H}$ & 4.57677833 & 6.34856147 & -0.87152806 \\
\hline $\mathrm{H}$ & -4.80128835 & -5.44184139 & -1.77884513 & $\mathrm{H}$ & 3.58185826 & 7.70486054 & -0.31382702 \\
\hline $\mathrm{H}$ & -5.78051144 & -4.18765330 & -1.02763408 & $\mathrm{H}$ & 2.86577321 & 6.42965344 & -1.30351509 \\
\hline $\mathrm{C}$ & 4.28350931 & -0.17850201 & 0.64260705 & $\mathrm{C}$ & 4.26209731 & 6.24351047 & 1.86515513 \\
\hline $\mathrm{C}$ & 4.79765635 & -1.46411411 & 0.92288007 & $\mathrm{H}$ & 4.05397529 & 5.75426340 & 2.82050220 \\
\hline $\mathrm{C}$ & 5.79253641 & -1.55519011 & 1.89422314 & $\mathrm{H}$ & 4.22183430 & 7.32368454 & 2.03147515 \\
\hline $\mathrm{H}$ & 6.17821042 & -2.54467819 & 2.11862115 & $\mathrm{H}$ & 5.28371738 & 5.99200544 & 1.56753211 \\
\hline $\mathrm{C}$ & 6.30350647 & -0.45094203 & 2.61424419 & $\mathrm{C}$ & 0.27620902 & 0.60755605 & -4.26882931 \\
\hline $\mathrm{C}$ & 5.77058842 & 0.80396406 & 2.33530917 & $\mathrm{C}$ & 0.61001504 & 0.00830600 & -5.64695742 \\
\hline $\mathrm{H}$ & 6.11838646 & 1.68362712 & 2.86206521 & $\mathrm{H}$ & 1.09374508 & 0.72226305 & -6.31504148 \\
\hline $\mathrm{C}$ & 4.77226234 & 0.94263707 & 1.36490510 & $\mathrm{H}$ & -0.32336402 & -0.30393002 & -6.12678646 \\
\hline $\mathrm{C}$ & 4.04245629 & 2.08027115 & 0.86363306 & $\mathrm{H}$ & 1.24608209 & -0.87712807 & -5.56370742 \\
\hline $\mathrm{C}$ & 4.08239429 & 3.44420625 & 1.17174209 & $\mathrm{C}$ & -0.56195704 & 1.88812414 & -4.44803932 \\
\hline $\mathrm{H}$ & 4.76889735 & 3.79259627 & 1.93292914 & $\mathrm{H}$ & -0.93855207 & 2.26334417 & -3.49542325 \\
\hline $\mathrm{C}$ & 3.24945124 & 4.33321331 & 0.50019304 & $\mathrm{H}$ & -1.43574510 & 1.65048212 & -5.06431336 \\
\hline $\mathrm{C}$ & 2.38294517 & 3.80456827 & -0.48447903 & $\mathrm{H}$ & -0.01788400 & 2.69044419 & -4.94672935 \\
\hline $\mathrm{H}$ & 1.72300412 & 4.48411332 & -1.01408107 & $\mathrm{C}$ & -0.57563504 & -0.42277703 & -3.50044525 \\
\hline $\mathrm{C}$ & 2.31342317 & 2.45533417 & -0.82786306 & $\mathrm{H}$ & -0.07175301 & -1.38917010 & -3.42504924 \\
\hline $\mathrm{C}$ & 3.16600323 & 1.57156211 & -0.13143501 & $\mathrm{H}$ & -1.51308411 & -0.57602104 & -4.04607329 \\
\hline $\mathrm{C}$ & 1.30675709 & 2.02461115 & -1.87532613 & $\mathrm{H}$ & -0.82246206 & -0.09810401 & -2.48966118 \\
\hline $\mathrm{H}$ & 0.84730006 & 2.91263221 & -2.31242216 & $\mathrm{C}$ & 3.21590223 & 1.78083613 & -4.26667231 \\
\hline $\mathrm{H}$ & 0.51016604 & 1.44536810 & -1.39072610 & $\mathrm{C}$ & 3.86805128 & 0.76823106 & -5.23145337 \\
\hline $\mathrm{C}$ & 4.25430931 & -2.73352619 & 0.30262402 & $\mathrm{H}$ & 4.25754030 & -0.10007401 & -4.69451834 \\
\hline $\mathrm{H}$ & 3.21538223 & -2.86553921 & 0.61858405 & $\mathrm{H}$ & 4.71235634 & 1.25002209 & -5.73601541 \\
\hline $\mathrm{H}$ & 4.81661135 & -3.59398426 & 0.67364105 & $\mathrm{H}$ & 3.18658123 & 0.40988503 & -6.00132441 \\
\hline $\mathrm{C}$ & 7.40028554 & -0.68041005 & 3.67101026 & $\mathrm{C}$ & 2.68247319 & 2.98918622 & -5.05476037 \\
\hline $\mathrm{C}$ & 6.87465351 & -1.63011012 & 4.77286734 & $\mathrm{H}$ & 1.99038214 & 2.70311019 & -5.84767340 \\
\hline $\mathrm{H}$ & 5.99850543 & -1.20190909 & 5.26733138 & $\mathrm{H}$ & 3.52451925 & 3.50633725 & -5.52820539 \\
\hline $\mathrm{H}$ & 7.64522957 & -1.80161113 & 5.53166341 & $\mathrm{H}$ & 2.18369616 & 3.71072827 & -4.40363232 \\
\hline $\mathrm{H}$ & 6.58517149 & -2.60209719 & 4.36660231 & $\mathrm{C}$ & 4.31014131 & 2.28024917 & -3.29998924 \\
\hline $\mathrm{C}$ & 7.84080459 & 0.63121705 & 4.34658431 & $\mathrm{H}$ & 3.96903629 & 3.11143322 & -2.68558220 \\
\hline $\mathrm{H}$ & 8.25030459 & 1.34188610 & 3.62358926 & $\mathrm{H}$ & 5.16337737 & 2.62588419 & -3.89386028 \\
\hline $\mathrm{H}$ & 8.62100759 & 0.42191003 & 5.08383436 & $\mathrm{H}$ & 4.66513633 & 1.49787311 & -2.62823019 \\
\hline $\mathrm{H}$ & 7.01251048 & 1.11557008 & 4.87062135 & $\mathrm{C}$ & 3.46407225 & -4.56637433 & -1.91238814 \\
\hline $\mathrm{C}$ & 8.64504362 & -1.31330009 & 3.00614722 & $\mathrm{C}$ & 2.30498517 & -4.83987735 & -0.92810007 \\
\hline $\mathrm{H}$ & 8.41313963 & -2.27654916 & 2.54555918 & $\mathrm{H}$ & 1.57924511 & -4.02613229 & -0.89190807 \\
\hline $\mathrm{H}$ & 9.43317169 & -1.48027511 & 3.74767427 & $\mathrm{H}$ & 2.66950219 & -5.01931236 & 0.08569701 \\
\hline $\mathrm{H}$ & 9.04376963 & -0.65778005 & 2.22701216 & $\mathrm{H}$ & 1.78896613 & -5.75033940 & -1.25193609 \\
\hline $\mathrm{C}$ & 3.23872723 & 5.84694340 & 0.78511106 & $\mathrm{C}$ & 2.88654821 & -4.57140833 & -3.34314324 \\
\hline $\mathrm{C}$ & 1.83713713 & 6.27655844 & 1.27705209 & $\mathrm{H}$ & 2.45766318 & -5.55660141 & -3.55547325 \\
\hline $\mathrm{H}$ & 1.06519508 & 6.06231944 & 0.53461704 & $\mathrm{H}$ & 3.63883126 & -4.36815631 & -4.10661430 \\
\hline $\mathrm{H}$ & 1.81406913 & 7.35199054 & 1.48162611 & $\mathrm{H}$ & 2.08624415 & -3.83465427 & -3.44632925 \\
\hline
\end{tabular}




\begin{tabular}{|c|c|c|c|c|c|c|c|}
\hline $\mathrm{C}$ & 4.49673932 & -5.69646338 & -1.75872613 & $\mathrm{C}$ & 5.56965900 & -0.89076800 & 0.21482000 \\
\hline $\mathrm{H}$ & 4.94659236 & -5.70629243 & -0.76316006 & $\mathrm{C}$ & 4.69129000 & 0.21314700 & 0.26530100 \\
\hline $\mathrm{H}$ & 5.29678935 & -5.64951642 & -2.49824218 & $\mathrm{C}$ & 5.29560700 & -2.18248800 & 0.95643600 \\
\hline $\mathrm{H}$ & 3.98766729 & -6.65736248 & -1.89237014 & $\mathrm{H}$ & 5.24761200 & -1.95116400 & 2.02431300 \\
\hline $\mathrm{C}$ & 5.92838542 & -2.60537019 & -2.21811116 & $\mathrm{H}$ & 6.13028000 & -2.87368400 & 0.82068400 \\
\hline $\mathrm{C}$ & 6.00484545 & -3.04126422 & -3.69370726 & $\mathrm{C}$ & 0.87740700 & 1.56316800 & 2.14835000 \\
\hline $\mathrm{H}$ & 5.25846038 & -2.53060318 & -4.30824731 & $\mathrm{H}$ & 0.15899100 & 1.18464200 & 1.41342400 \\
\hline $\mathrm{H}$ & 5.87933445 & -4.11619729 & -3.82700428 & $\mathrm{H}$ & 0.33328400 & 2.27812600 & 2.77066500 \\
\hline $\mathrm{H}$ & 6.99098852 & -2.77665420 & -4.08985529 & $\mathrm{C}$ & 2.70010000 & 5.98615500 & 0.54296000 \\
\hline $\mathrm{C}$ & 6.26813648 & -1.10179608 & -2.15442116 & $\mathrm{C}$ & 3.83885900 & 6.67778600 & -0.22925200 \\
\hline $\mathrm{H}$ & 7.28378055 & -0.95401207 & -2.53780718 & $\mathrm{H}$ & 4.80916400 & 6.51947800 & 0.24913900 \\
\hline $\mathrm{H}$ & 6.23168643 & -0.70928305 & -1.13901408 & $\mathrm{H}$ & 3.65865500 & 7.75598600 & -0.26214200 \\
\hline $\mathrm{H}$ & 5.59279841 & -0.51118604 & -2.77486220 & $\mathrm{H}$ & 3.90406500 & 6.32104200 & -1.26078100 \\
\hline $\mathrm{C}$ & 6.97159952 & -3.37150124 & -1.38418910 & $\mathrm{C}$ & 2.66386900 & 6.58133400 & 1.97016600 \\
\hline $\mathrm{H}$ & 6.81441451 & -4.44930332 & -1.39101710 & $\mathrm{H}$ & 1.84421300 & 6.16755100 & 2.56199700 \\
\hline $\mathrm{H}$ & 6.98562648 & -3.02709622 & -0.34920102 & $\mathrm{H}$ & 2.52965800 & 7.66717800 & 1.92898200 \\
\hline $\mathrm{H}$ & 7.96652758 & -3.18198223 & -1.80241313 & $\mathrm{H}$ & 3.59709500 & 6.37364400 & 2.50088300 \\
\hline $\mathrm{C}$ & -0.04770000 & -1.46663710 & -0.14920401 & $\mathrm{C}$ & 1.37126800 & 6.31323000 & -0.17766400 \\
\hline & & & & $\mathrm{H}$ & 1.37943800 & 5.92019900 & -1.19825300 \\
\hline \multirow{2}{*}{\multicolumn{4}{|c|}{$\left.\left[{ }^{(\mathrm{Bu}}(\mathrm{PNP}) \mathrm{Fe}\right)_{2} \mathrm{~S}\right](8)$, 9et }} & $\mathrm{H}$ & 1.22013100 & 7.39639400 & -0.23110900 \\
\hline & & & & $\mathrm{H}$ & 0.51183300 & 5.88110400 & 0.33963500 \\
\hline $\mathrm{Fe}$ & 2.02077600 & -1.16251300 & 0.91473300 & $\mathrm{C}$ & 8.59969300 & 0.61821300 & -1.64917600 \\
\hline $\mathrm{Fe}$ & -1.73812200 & -0.91398900 & -1.30834300 & $\mathrm{C}$ & 8.66608000 & -0.38425600 & -2.82495000 \\
\hline S & 0.23051000 & -1.73175300 & -0.42781800 & $\mathrm{H}$ & 8.51830500 & -1.41334100 & -2.48921400 \\
\hline $\mathrm{P}$ & 3.67179400 & -3.03232600 & 0.59130300 & $\mathrm{H}$ & 9.64159200 & -0.33156600 & -3.31939100 \\
\hline $\mathrm{P}$ & 1.29341100 & 0.02730100 & 3.15513300 & $\mathrm{H}$ & 7.89527500 & -0.16220800 & -3.56808100 \\
\hline $\mathrm{P}$ & -1.23741400 & 0.91134400 & -3.06064500 & $\mathrm{C}$ & 9.71205200 & 0.28034100 & -0.62902500 \\
\hline $\mathrm{P}$ & -3.34931000 & -2.95866300 & -1.48817900 & $\mathrm{H}$ & 9.69441400 & 0.98188100 & 0.20945600 \\
\hline $\mathrm{N}$ & 3.41978300 & 0.29402500 & 0.81812100 & $\mathrm{H}$ & 10.69753400 & 0.33865300 & -1.10270400 \\
\hline $\mathrm{N}$ & -3.13113700 & 0.16519600 & -0.32820400 & $\mathrm{H}$ & 9.59754700 & -0.72717400 & -0.22246500 \\
\hline $\mathrm{C}$ & 3.09231900 & 1.64517600 & 0.80935600 & $\mathrm{C}$ & 8.88215100 & 2.02403900 & -2.20991400 \\
\hline $\mathrm{C}$ & 1.99388100 & 2.28702000 & 1.41731100 & $\mathrm{H}$ & 8.13809500 & 2.31911800 & -2.95479000 \\
\hline $\mathrm{C}$ & 1.92955200 & 3.67481900 & 1.29811300 & $\mathrm{H}$ & 9.86200300 & 2.03730900 & -2.69542800 \\
\hline $\mathrm{H}$ & 1.08682600 & 4.17441800 & 1.76482700 & $\mathrm{H}$ & 8.89476400 & 2.78069500 & -1.42091400 \\
\hline $\mathrm{C}$ & 2.88892600 & 4.45905500 & 0.61675500 & $\mathrm{C}$ & 3.61441100 & -4.47731000 & 1.85481800 \\
\hline $\mathrm{C}$ & 3.98302200 & 3.80530200 & 0.05692500 & $\mathrm{C}$ & 2.19028800 & -5.07052000 & 1.84485700 \\
\hline $\mathrm{H}$ & 4.76313200 & 4.36122800 & -0.44742200 & $\mathrm{H}$ & 1.43764700 & -4.30317500 & 2.03780200 \\
\hline $\mathrm{C}$ & 4.09722500 & 2.41537200 & 0.16583800 & $\mathrm{H}$ & 2.11163800 & -5.82799400 & 2.63261200 \\
\hline $\mathrm{C}$ & 5.13084200 & 1.47915000 & -0.20511800 & $\mathrm{H}$ & 1.94030000 & -5.54975300 & 0.89929500 \\
\hline $\mathrm{C}$ & 6.37373600 & 1.62558100 & -0.83201400 & $\mathrm{C}$ & 3.85345400 & -3.86922000 & 3.25319000 \\
\hline $\mathrm{H}$ & 6.66889000 & 2.60222300 & -1.19436400 & $\mathrm{H}$ & 4.88969600 & -3.56017400 & 3.40242900 \\
\hline C & 7.22096300 & 0.52994600 & -0.96788300 & $\mathrm{H}$ & 3.62633300 & -4.62565200 & 4.01173800 \\
\hline $\mathrm{C}$ & 6.79817800 & -0.70043500 & -0.41277100 & $\mathrm{H}$ & 3.20335400 & -3.01003100 & 3.43759500 \\
\hline $\mathrm{H}$ & 7.47916200 & -1.54516300 & -0.44148200 & $\mathrm{C}$ & 4.64630300 & -5.59447900 & 1.62396100 \\
\hline
\end{tabular}




\begin{tabular}{|c|c|c|c|c|c|c|c|}
\hline $\mathrm{H}$ & 4.45791800 & -6.14929600 & 0.70391000 & $\mathrm{C}$ & -5.54229400 & -1.86891600 & 1.69535100 \\
\hline $\mathrm{H}$ & 4.59144100 & -6.31110800 & 2.45148300 & $\mathrm{H}$ & -5.77077300 & -2.89436900 & 1.95823600 \\
\hline $\mathrm{H}$ & 5.67011900 & -5.21318200 & 1.59271500 & $\mathrm{C}$ & -6.34391300 & -0.83583000 & 2.21868500 \\
\hline $\mathrm{C}$ & 3.87539000 & -3.68214700 & -1.20428000 & $\mathrm{C}$ & -6.03200100 & 0.47065500 & 1.84384900 \\
\hline $\mathrm{C}$ & 3.62337100 & -2.47621200 & -2.13571100 & $\mathrm{H}$ & -6.62705500 & 1.30343500 & 2.20354800 \\
\hline $\mathrm{H}$ & 2.61138100 & -2.08817800 & -2.01232400 & $\mathrm{C}$ & -4.95193500 & 0.72101000 & 0.99808900 \\
\hline $\mathrm{H}$ & 3.73703000 & -2.80899300 & -3.17400400 & $\mathrm{C}$ & -4.42231200 & 1.93534900 & 0.43301300 \\
\hline $\mathrm{H}$ & 4.33529600 & -1.66753200 & -1.96577200 & $\mathrm{C}$ & -4.82255700 & 3.27112000 & 0.53789900 \\
\hline $\mathrm{C}$ & 2.80913900 & -4.74924500 & -1.51233300 & $\mathrm{H}$ & -5.68017500 & 3.51606000 & 1.15155300 \\
\hline $\mathrm{H}$ & 2.98805600 & -5.68639400 & -0.98135800 & $\mathrm{C}$ & -4.12527000 & 4.26139100 & -0.14427600 \\
\hline $\mathrm{H}$ & 2.83862900 & -4.97361300 & -2.58430300 & $\mathrm{C}$ & -3.00968000 & 3.85942400 & -0.91212000 \\
\hline $\mathrm{H}$ & 1.80489600 & -4.39056800 & -1.27728300 & $\mathrm{H}$ & -2.43464100 & 4.62258400 & -1.42608100 \\
\hline $\mathrm{C}$ & 5.27842800 & -4.24335700 & -1.50115200 & $\mathrm{C}$ & -2.56776800 & 2.54294300 & -1.03827500 \\
\hline $\mathrm{H}$ & 6.05005200 & -3.47796200 & -1.40491200 & $\mathrm{C}$ & -3.30823100 & 1.54679200 & -0.35811000 \\
\hline $\mathrm{H}$ & 5.30133100 & -4.59256200 & -2.53951100 & $\mathrm{C}$ & -1.27844800 & 2.29206700 & -1.79316300 \\
\hline $\mathrm{H}$ & 5.54714200 & -5.08903100 & -0.86767100 & $\mathrm{H}$ & -0.50514400 & 1.99584900 & -1.07809000 \\
\hline $\mathrm{C}$ & -0.41382500 & -0.42908900 & 3.92135700 & $\mathrm{H}$ & -0.93952100 & 3.22731700 & -2.24437100 \\
\hline $\mathrm{C}$ & -0.32516400 & -1.88053100 & 4.43903100 & $\mathrm{C}$ & -3.69035900 & -2.87967600 & 0.35588300 \\
\hline $\mathrm{H}$ & -0.06200300 & -2.56852500 & 3.63199700 & $\mathrm{H}$ & -4.20137100 & -3.78557700 & 0.69152100 \\
\hline $\mathrm{H}$ & -1.30443600 & -2.18201800 & 4.82631600 & $\mathrm{H}$ & -2.69104500 & -2.89429300 & 0.80269200 \\
\hline $\mathrm{H}$ & 0.39960300 & -2.00603400 & 5.24409800 & $\mathrm{C}$ & -7.53334600 & -1.10499900 & 3.16146800 \\
\hline $\mathrm{C}$ & -0.90745300 & 0.50160900 & 5.04353600 & $\mathrm{C}$ & -7.30950100 & -0.36824800 & 4.50251500 \\
\hline $\mathrm{H}$ & -0.29193300 & 0.45724700 & 5.94221800 & $\mathrm{H}$ & -7.21789700 & 0.71077500 & 4.35992200 \\
\hline $\mathrm{H}$ & -1.92152100 & 0.19956100 & 5.32770300 & $\mathrm{H}$ & -8.14833200 & -0.54636900 & 5.18342500 \\
\hline $\mathrm{H}$ & -0.96351400 & 1.54181900 & 4.71473200 & $\mathrm{H}$ & -6.39512300 & -0.71878500 & 4.98903700 \\
\hline $\mathrm{C}$ & -1.46159500 & -0.40108000 & 2.79185800 & $\mathrm{C}$ & -8.83840700 & -0.58940000 & 2.51105200 \\
\hline $\mathrm{H}$ & -1.71038000 & 0.61408900 & 2.47906000 & $\mathrm{H}$ & -8.79003400 & 0.48200500 & 2.30364800 \\
\hline $\mathrm{H}$ & -2.38677900 & -0.85653200 & 3.15875200 & $\mathrm{H}$ & -9.03002300 & -1.10283300 & 1.56469400 \\
\hline $\mathrm{H}$ & -1.13651000 & -0.95819500 & 1.91198700 & $\mathrm{H}$ & -9.69312400 & -0.76425600 & 3.17290200 \\
\hline $\mathrm{C}$ & 2.49063500 & 0.62765900 & 4.54884700 & $\mathrm{C}$ & -7.71741700 & -2.60233700 & 3.47032000 \\
\hline $\mathrm{C}$ & 3.92601900 & 0.66080000 & 3.98459000 & $\mathrm{H}$ & -7.91646400 & -3.18338200 & 2.56562100 \\
\hline $\mathrm{H}$ & 4.23331800 & -0.31192000 & 3.59835100 & $\mathrm{H}$ & -6.83885100 & -3.02682100 & 3.96402700 \\
\hline $\mathrm{H}$ & 4.60974000 & 0.93371000 & 4.79654900 & $\mathrm{H}$ & -8.57022200 & -2.73665000 & 4.14171400 \\
\hline $\mathrm{H}$ & 4.04458000 & 1.39099000 & 3.18661900 & $\mathrm{C}$ & -4.50422300 & 5.75261300 & -0.08188000 \\
\hline $\mathrm{C}$ & 2.14733600 & 2.03782600 & 5.06458100 & $\mathrm{C}$ & -4.79858600 & 6.27912700 & -1.50590400 \\
\hline $\mathrm{H}$ & 2.20438400 & 2.78114100 & 4.26744000 & $\mathrm{H}$ & -3.93220000 & 6.17651500 & -2.16350700 \\
\hline $\mathrm{H}$ & 2.88016800 & 2.32361000 & 5.82807000 & $\mathrm{H}$ & -5.06856300 & 7.33971700 & -1.47367900 \\
\hline $\mathrm{H}$ & 1.16021200 & 2.09561800 & 5.52196800 & $\mathrm{H}$ & -5.62845700 & 5.72981700 & -1.95894700 \\
\hline $\mathrm{C}$ & 2.48847600 & -0.37533900 & 5.71936900 & $\mathrm{C}$ & -3.33639500 & 6.56278000 & 0.52809400 \\
\hline $\mathrm{H}$ & 1.54192800 & -0.40277700 & 6.25837200 & $\mathrm{H}$ & -3.11174400 & 6.21537300 & 1.54032300 \\
\hline $\mathrm{H}$ & 3.26284300 & -0.07903200 & 6.43502500 & $\mathrm{H}$ & -3.59304800 & 7.62589000 & 0.58159300 \\
\hline $\mathrm{H}$ & 2.72430300 & -1.38984800 & 5.38700900 & $\mathrm{H}$ & -2.42451900 & 6.46980400 & -0.06618100 \\
\hline $\mathrm{C}$ & -4.13659900 & -0.33305900 & 0.49944900 & $\mathrm{C}$ & -5.75513600 & 5.99864800 & 0.78170900 \\
\hline $\mathrm{C}$ & -4.45398100 & -1.66502900 & 0.84094300 & $\mathrm{H}$ & -6.62835100 & 5.46978300 & 0.39002900 \\
\hline
\end{tabular}




$\begin{array}{lrrrllll}\mathrm{H} & -5.99140300 & 7.06649200 & 0.79175300 & \mathrm{H} & -6.59552200 & -1.54897300 & -2.83120800 \\ \mathrm{H} & -5.60155200 & 5.68480800 & 1.81774400 & \mathrm{H} & -5.06892600 & -0.79653300 & -2.35725200 \\ \mathrm{C} & 0.56701200 & 1.00227300 & -3.73207900 & \mathrm{C} & -6.06019100 & -4.04735700 & -1.75387200 \\ \mathrm{C} & 0.87040900 & -0.34386200 & -4.42193100 & \mathrm{H} & -5.77256400 & -5.07541200 & -1.95942300 \\ \mathrm{H} & 0.75588400 & -1.17460900 & -3.72292300 & \mathrm{H} & -7.04512800 & -3.88639600 & -2.20702400 \\ \mathrm{H} & 1.90789900 & -0.34170800 & -4.77339500 & \mathrm{H} & -6.18301500 & -3.92836600 & -0.67524400 \\ \mathrm{H} & 0.22995100 & -0.52860000 & -5.28569300 & \mathrm{C} & -4.87497100 & -3.24214800 & -3.83963200 \\ \mathrm{C} & 0.84678900 & 2.16389400 & -4.70269900 & \mathrm{H} & -4.20857200 & -2.49334500 & -4.27649200 \\ \mathrm{H} & 0.29654800 & 2.08689800 & -5.64072400 & \mathrm{H} & -5.84021300 & -3.16375600 & -4.35130100 \\ \mathrm{H} & 1.91369200 & 2.15560200 & -4.95171100 & \mathrm{H} & -4.46717800 & -4.22912500 & -4.06409200\end{array}$

H $\begin{array}{llll}0.63143500 & 3.13588000 & -4.25297200\end{array}$

$\begin{array}{llll}1.52793100 & 1.15780900 & -2.53628100\end{array}$

$\begin{array}{llll}1.47360200 & 2.15000600 & -2.08573800\end{array}$

$\begin{array}{llll}2.55350300 & 1.02554100 & -2.89594800\end{array}$

$\begin{array}{llll}1.34947400 & 0.40906400 & -1.76440200\end{array}$

$\begin{array}{llll}-2.46695500 & 1.44878200 & -4.43812700\end{array}$

$\begin{array}{llll}-2.44106500 & 2.95794300 & -4.74396600\end{array}$

$-2.72862500 \quad 3.54694600 \quad-3.87154200$

$\begin{array}{llll}-3.17369300 & 3.17007700 & -5.53128600\end{array}$

$\begin{array}{llll}-1.47306300 & 3.31008400 & -5.09664600\end{array}$

$\begin{array}{llll}-3.87849000 & 1.08473600 & -3.94184800\end{array}$

$\begin{array}{llll}-3.97763800 & 0.00739700 & -3.81483800\end{array}$

$\begin{array}{llll}-4.61372500 & 1.40093700 & -4.69055600\end{array}$

$\begin{array}{lll}-4.13148900 & 1.57019300 & -2.99890900\end{array}$

$\begin{array}{llll}-2.21174300 & 0.63803400 & -5.72349100\end{array}$

$\begin{array}{llll}-1.28104900 & 0.90816000 & -6.22252800\end{array}$

$\begin{array}{llll}-3.02780600 & 0.82820300 & -6.42925500\end{array}$

$-2.19405500 \quad-0.43696700 \quad-5.52422500$

$\begin{array}{llll}-2.44600500 & -4.65291800 & -1.73001700\end{array}$

$-3.35491400 \quad-5.88334600 \quad-1.89244500$

$\begin{array}{llll}-4.00637900 & -6.03075400 & -1.02865100\end{array}$

$-2.71816000 \quad-6.77161800-1.97293600$

$-3.96968700 \quad-5.84864600 \quad-2.79178000$

$\begin{array}{llll}-1.55998800 & -4.50239500 & -2.98519600\end{array}$

$\begin{array}{llll}-2.14293200 & -4.27990100 & -3.88198500\end{array}$

$\begin{array}{lll}-1.02146100 & -5.43978900 & -3.16466900\end{array}$

$-0.82025900 \quad-3.71070100-2.85142500$

$\begin{array}{lll}-1.53178800 & -4.92138600 & -0.51705900\end{array}$

$\begin{array}{lll}-0.86092000 & -4.08696800 & -0.30665000\end{array}$

$\begin{array}{lll}-0.91687400 & -5.80030900 & -0.74215400\end{array}$

$-2.10751200 \quad-5.15173000 \quad 0.38255500$

$\begin{array}{llll}-5.08153100 & -3.00539500 & -2.33007300\end{array}$

$\begin{array}{lll}-5.74441400 & -1.62166500 & -2.14475600\end{array}$

$\begin{array}{llll}-6.12074500 & -1.48826600 & -1.13212700\end{array}$

\section{$\left[\left({ }^{t \mathrm{Bu}}(\mathrm{PNP}) \mathrm{Fe}\right) \mathrm{S}\right](8)$, 1et}

$\mathrm{Fe}$

$\mathrm{Fe}$

S

$P$

P

$\mathrm{P}$

$\mathrm{P}$

$\mathrm{N}$

$\mathrm{N}$

$\mathrm{C}$

$\mathrm{C}$

C

$\mathrm{H}$

C

C

$\mathrm{H}$

$\mathrm{C}$

C

C

$\mathrm{H}$

C

C

$\mathrm{H}$

C

C

C

$\mathrm{H}$

$\mathrm{H}$

C

$\mathrm{H}$

$\mathrm{H}$

$\begin{array}{rrr}2.00534900 & -1.15472500 & 0.91743300 \\ -1.72367600 & -0.90252600 & -1.31042400 \\ 0.23248200 & -1.64679300 & -0.42106100 \\ 3.64141700 & -3.02559200 & 0.57161100 \\ 1.28770700 & 0.01811600 & 3.16289500 \\ -1.21800700 & 0.91669200 & -3.05425800 \\ -3.31743200 & -2.96036200 & -1.48042600 \\ 3.41052300 & 0.29676200 & 0.82560000 \\ -3.12465800 & 0.17244300 & -0.33473100 \\ 3.08933200 & 1.64926000 & 0.82763500 \\ 1.99664900 & 2.29181800 & 1.44516700 \\ 1.93824900 & 3.68071900 & 1.33636000 \\ 1.10006600 & 4.18088900 & 1.81061800 \\ 2.89801400 & 4.46548500 & 0.65614800 \\ 3.98690400 & 3.81091200 & 0.08717100 \\ 4.76738900 & 4.36698100 & -0.41645300 \\ 4.09539900 & 2.41973400 & 0.18577300 \\ 5.12356200 & 1.48146200 & -0.19521700 \\ 6.36570500 & 1.62548200 & -0.82428400 \\ 6.66501500 & 2.60282500 & -1.18133200 \\ 7.20742800 & 0.52655900 & -0.96851300 \\ 6.78031600 & -0.70521500 & -0.41952800 \\ 7.45737000 & -1.55289400 & -0.45444000 \\ 5.55200700 & -0.89317100 & 0.20905600 \\ 4.67926300 & 0.21450100 & 0.26806400 \\ 5.27015200 & -2.18743700 & 0.94303300 \\ 5.22284400 & -1.96213300 & 2.01220100 \\ 6.10058300 & -2.88295800 & 0.80368800 \\ 0.87925800 & 1.56856800 & 2.17584000 \\ 0.15257600 & 1.20518300 & 1.44167900 \\ 0.34623400 & 2.28088200 & 2.81074300 \\ \end{array}$

S46 


\begin{tabular}{|c|c|c|c|c|c|c|c|}
\hline $\mathrm{C}$ & 2.71492700 & 5.99374000 & 0.59295400 & $\mathrm{C}$ & 2.77737900 & -4.72471300 & -1.54634700 \\
\hline $\mathrm{C}$ & 3.85244000 & 6.68578400 & -0.18073100 & $\mathrm{H}$ & 2.95250700 & -5.66646200 & -1.02233500 \\
\hline $\mathrm{H}$ & 4.82450400 & 6.52133700 & 0.29200100 & $\mathrm{H}$ & 2.80622700 & -4.94094900 & -2.61998800 \\
\hline $\mathrm{H}$ & 3.67588300 & 7.76478600 & -0.20653100 & $\mathrm{H}$ & 1.77412900 & -4.36549700 & -1.30856700 \\
\hline $\mathrm{H}$ & 3.91142400 & 6.33478200 & -1.21460700 & $\mathrm{C}$ & 5.24779600 & -4.22877000 & -1.52804900 \\
\hline $\mathrm{C}$ & 2.68820400 & 6.58013800 & 2.02398700 & $\mathrm{H}$ & 6.02251400 & -3.46750200 & -1.42487800 \\
\hline $\mathrm{H}$ & 1.86997400 & 6.16571000 & 2.61736000 & $\mathrm{H}$ & 5.27057000 & -4.56984200 & -2.56912900 \\
\hline $\mathrm{H}$ & 2.55789600 & 7.66672700 & 1.99028800 & $\mathrm{H}$ & 5.51219500 & -5.08050400 & -0.90103400 \\
\hline $\mathrm{H}$ & 3.62331700 & 6.36553700 & 2.54860800 & $\mathrm{C}$ & -0.42016000 & -0.43311000 & 3.93111700 \\
\hline $\mathrm{C}$ & 1.38372700 & 6.33033200 & -0.11889300 & $\mathrm{C}$ & -0.34076300 & -1.89005100 & 4.43452300 \\
\hline $\mathrm{H}$ & 1.38439100 & 5.94168200 & -1.14118600 & $\mathrm{H}$ & -0.08375400 & -2.57178600 & 3.62016700 \\
\hline $\mathrm{H}$ & 1.23731200 & 7.41440500 & -0.16680100 & $\mathrm{H}$ & -1.32130800 & -2.18857400 & 4.82091100 \\
\hline $\mathrm{H}$ & 0.52507300 & 5.89998100 & 0.40116500 & $\mathrm{H}$ & 0.38484300 & -2.02857600 & 5.23667300 \\
\hline $\mathrm{C}$ & 8.58530000 & 0.61261000 & -1.65187900 & $\mathrm{C}$ & -0.90367800 & 0.49054400 & 5.06355700 \\
\hline $\mathrm{C}$ & 8.64687500 & -0.38608300 & -2.83110500 & $\mathrm{H}$ & -0.28616800 & 0.43306500 & 5.96007600 \\
\hline $\mathrm{H}$ & 8.49659900 & -1.41587800 & -2.49869300 & $\mathrm{H}$ & -1.91922600 & 0.19354900 & 5.34777600 \\
\hline $\mathrm{H}$ & 9.62177700 & -0.33460200 & -3.32687700 & $\mathrm{H}$ & -0.95268800 & 1.53424600 & 4.74483400 \\
\hline $\mathrm{H}$ & 7.87558700 & -0.15918100 & -3.57227300 & $\mathrm{C}$ & -1.47142800 & -0.38644100 & 2.80561100 \\
\hline $\mathrm{C}$ & 9.69813200 & 0.26780200 & -0.63454000 & $\mathrm{H}$ & -1.71582500 & 0.63351600 & 2.50520200 \\
\hline $\mathrm{H}$ & 9.68410200 & 0.96671900 & 0.20619900 & $\mathrm{H}$ & -2.39780000 & -0.84169600 & 3.16951700 \\
\hline $\mathrm{H}$ & 10.68314400 & 0.32425200 & -1.10946600 & $\mathrm{H}$ & -1.15152000 & -0.93413500 & 1.91827200 \\
\hline $\mathrm{H}$ & 9.58071200 & -0.74059600 & -0.23096600 & $\mathrm{C}$ & 2.49446900 & 0.59377700 & 4.55881000 \\
\hline $\mathrm{C}$ & 8.87140900 & 2.01932500 & -2.20854300 & $\mathrm{C}$ & 3.92871300 & 0.61996700 & 3.99149800 \\
\hline $\mathrm{H}$ & 8.12678600 & 2.31935200 & -2.95087900 & $\mathrm{H}$ & 4.22833100 & -0.35251000 & 3.59858600 \\
\hline $\mathrm{H}$ & 9.85032300 & 2.03090000 & -2.69600700 & $\mathrm{H}$ & 4.61608700 & 0.88274200 & 4.80373000 \\
\hline $\mathrm{H}$ & 8.88822500 & 2.77336900 & -1.41714200 & $\mathrm{H}$ & 4.05065500 & 1.35414100 & 3.19781200 \\
\hline $\mathrm{C}$ & 3.58259500 & -4.48194300 & 1.82363100 & $\mathrm{C}$ & 2.16506700 & 2.00208100 & 5.08870600 \\
\hline $\mathrm{C}$ & 2.15839200 & -5.07443300 & 1.80965800 & $\mathrm{H}$ & 2.23001800 & 2.75272900 & 4.29912400 \\
\hline $\mathrm{H}$ & 1.40663400 & -4.30819100 & 2.01031800 & $\mathrm{H}$ & 2.90055600 & 2.27271500 & 5.85513500 \\
\hline $\mathrm{H}$ & 2.07986200 & -5.83868600 & 2.59085900 & $\mathrm{H}$ & 1.17841900 & 2.06529900 & 5.54648600 \\
\hline $\mathrm{H}$ & 1.90757800 & -5.54561100 & 0.86023000 & $\mathrm{C}$ & 2.48633600 & -0.41994500 & 5.71998300 \\
\hline $\mathrm{C}$ & 3.82204200 & -3.88552200 & 3.22692900 & $\mathrm{H}$ & 1.54159800 & -0.44295000 & 6.26236000 \\
\hline $\mathrm{H}$ & 4.85830500 & -3.57771800 & 3.37850600 & $\mathrm{H}$ & 3.26620600 & -0.13798900 & 6.43546100 \\
\hline $\mathrm{H}$ & 3.59503100 & -4.64815500 & 3.97930400 & $\mathrm{H}$ & 2.71076400 & -1.43356400 & 5.37736400 \\
\hline $\mathrm{H}$ & 3.17196300 & -3.02789000 & 3.41844500 & $\mathrm{C}$ & -4.12787800 & -0.33028300 & 0.49248400 \\
\hline $\mathrm{C}$ & 4.61347500 & -5.59820600 & 1.58420700 & $\mathrm{C}$ & -4.43498100 & -1.66317500 & 0.84030300 \\
\hline $\mathrm{H}$ & 4.42445700 & -6.14576600 & 0.65999300 & $\mathrm{C}$ & -5.52337700 & -1.87245400 & 1.69329000 \\
\hline $\mathrm{H}$ & 4.55809700 & -6.32101700 & 2.40627900 & $\mathrm{H}$ & -5.74309000 & -2.89851000 & 1.96131200 \\
\hline $\mathrm{H}$ & 5.63762300 & -5.21766400 & 1.55570000 & $\mathrm{C}$ & -6.33566200 & -0.84387600 & 2.20903500 \\
\hline $\mathrm{C}$ & 3.84670100 & -3.66376000 & -1.22877500 & $\mathrm{C}$ & -6.03457200 & 0.46325400 & 1.82764600 \\
\hline $\mathrm{C}$ & 3.60238500 & -2.45053900 & -2.15251400 & $\mathrm{H}$ & -6.63809100 & 1.29253500 & 2.18128900 \\
\hline $\mathrm{H}$ & 2.59360900 & -2.05581400 & -2.02678800 & $\mathrm{C}$ & -4.95451600 & 0.71894900 & 0.98345400 \\
\hline $\mathrm{H}$ & 3.71450200 & -2.77691700 & -3.19297700 & $\mathrm{C}$ & -4.43436900 & 1.93488600 & 0.41341200 \\
\hline $\mathrm{H}$ & 4.31871500 & -1.64715300 & -1.97639300 & $\mathrm{C}$ & -4.84778200 & 3.26741800 & 0.50886900 \\
\hline
\end{tabular}




\begin{tabular}{|c|c|c|c|c|c|c|c|}
\hline $\mathrm{H}$ & -5.70973600 & 3.50784000 & 1.11824600 & $\mathrm{H}$ & 0.29906900 & 2.10338000 & -5.63890600 \\
\hline $\mathrm{C}$ & -4.15775300 & 4.26005200 & -0.17712700 & $\mathrm{H}$ & 1.91312000 & 2.20794100 & -4.94731600 \\
\hline $\mathrm{C}$ & -3.03542500 & 3.86403400 & -0.93836900 & $\mathrm{H}$ & 0.61073400 & 3.16778200 & -4.25755900 \\
\hline $\mathrm{H}$ & -2.46582200 & 4.62951200 & -1.45498300 & $\mathrm{C}$ & 1.54178400 & 1.21905700 & -2.52646300 \\
\hline $\mathrm{C}$ & -2.58025200 & 2.55123000 & -1.05463900 & $\mathrm{H}$ & 1.46801500 & 2.21355600 & -2.08378500 \\
\hline $\mathrm{C}$ & -3.31402800 & 1.55193900 & -0.37176700 & $\mathrm{H}$ & 2.57065100 & 1.10297700 & -2.88225300 \\
\hline $\mathrm{C}$ & -1.28444600 & 2.30889400 & -1.80097400 & $\mathrm{H}$ & 1.37540600 & 0.47437400 & -1.74850600 \\
\hline $\mathrm{H}$ & -0.51169300 & 2.02823700 & -1.07903100 & $\mathrm{C}$ & -2.45270300 & 1.41895300 & -4.44020900 \\
\hline $\mathrm{H}$ & -0.95454800 & 3.24416100 & -2.25902200 & $\mathrm{C}$ & -2.45607900 & 2.92534000 & -4.76038800 \\
\hline $\mathrm{C}$ & -3.65974400 & -2.87377200 & 0.36334900 & $\mathrm{H}$ & -2.75795200 & 3.51647700 & -3.89429100 \\
\hline $\mathrm{H}$ & -4.16318700 & -3.78223200 & 0.70361800 & $\mathrm{H}$ & -3.19052200 & 3.11527700 & -5.55171000 \\
\hline $\mathrm{H}$ & -2.66127300 & -2.87689100 & 0.81197700 & $\mathrm{H}$ & -1.49436800 & 3.29355500 & -5.11378700 \\
\hline $\mathrm{C}$ & -7.52437700 & -1.11843800 & 3.15117200 & $\mathrm{C}$ & -3.85773000 & 1.03233800 & -3.94311900 \\
\hline $\mathrm{C}$ & -7.30768000 & -0.37516600 & 4.48980800 & $\mathrm{H}$ & -3.93255100 & -0.04460000 & -3.79696500 \\
\hline $\mathrm{H}$ & -7.22330700 & 0.70389700 & 4.34315200 & $\mathrm{H}$ & -4.59710100 & 1.31871000 & -4.69972300 \\
\hline $\mathrm{H}$ & -8.14624700 & -0.55654500 & 5.17019700 & $\mathrm{H}$ & -4.12460400 & 1.52899800 & -3.00979700 \\
\hline $\mathrm{H}$ & -6.39160400 & -0.71748600 & 4.97898800 & $\mathrm{C}$ & -2.17884400 & 0.60122500 & -5.71730800 \\
\hline $\mathrm{C}$ & -8.83253200 & -0.61489300 & 2.49752800 & $\mathrm{H}$ & -1.25352100 & 0.88637700 & -6.21804900 \\
\hline $\mathrm{H}$ & -8.79194300 & 0.45606800 & 2.28621100 & $\mathrm{H}$ & -2.99782700 & 0.76697000 & -6.42587200 \\
\hline $\mathrm{H}$ & -9.01946100 & -1.13318000 & 1.55287500 & $\mathrm{H}$ & -2.13850400 & -0.47111300 & -5.50735200 \\
\hline $\mathrm{H}$ & -9.68658400 & -0.79359300 & 3.15921400 & $\mathrm{C}$ & -2.39571700 & -4.64558400 & -1.71148800 \\
\hline $\mathrm{C}$ & -7.69780400 & -2.61596900 & 3.46526000 & $\mathrm{C}$ & -3.28892300 & -5.88978900 & -1.85661200 \\
\hline $\mathrm{H}$ & -7.89086200 & -3.20186400 & 2.56238100 & $\mathrm{H}$ & -3.93682200 & -6.03543300 & -0.98984800 \\
\hline $\mathrm{H}$ & -6.81697400 & -3.03202800 & 3.96210900 & $\mathrm{H}$ & -2.64120700 & -6.77086100 & -1.92849600 \\
\hline $\mathrm{H}$ & -8.55073900 & -2.75419900 & 4.13570500 & $\mathrm{H}$ & -3.90603800 & -5.87314600 & -2.75495300 \\
\hline $\mathrm{C}$ & -4.55116000 & 5.74790400 & -0.12535600 & $\mathrm{C}$ & -1.51881400 & -4.49743800 & -2.97332900 \\
\hline $\mathrm{C}$ & -4.84382500 & 6.26331300 & -1.55377300 & $\mathrm{H}$ & -2.11048100 & -4.29788700 & -3.86971800 \\
\hline $\mathrm{H}$ & -3.97319400 & 6.16577100 & -2.20650800 & $\mathrm{H}$ & -0.96486100 & -5.42767500 & -3.14273000 \\
\hline $\mathrm{H}$ & -5.12478200 & 7.32125400 & -1.52895500 & $\mathrm{H}$ & -0.79313000 & -3.69032800 & -2.85608600 \\
\hline $\mathrm{H}$ & -5.66577600 & 5.70290700 & -2.00767900 & $\mathrm{C}$ & -1.47156000 & -4.88848900 & -0.50099400 \\
\hline $\mathrm{C}$ & -3.39400100 & 6.57276000 & 0.48538100 & $\mathrm{H}$ & -0.81411400 & -4.04111100 & -0.30100200 \\
\hline $\mathrm{H}$ & -3.17114500 & 6.23353700 & 1.50076000 & $\mathrm{H}$ & -0.84306700 & -5.75901300 & -0.72049300 \\
\hline $\mathrm{H}$ & -3.66084700 & 7.63373800 & 0.53114900 & $\mathrm{H}$ & -2.03891000 & -5.11922600 & 0.40372500 \\
\hline $\mathrm{H}$ & -2.47828700 & 6.48466500 & -0.10373900 & $\mathrm{C}$ & -5.05025300 & -3.03314500 & -2.31940600 \\
\hline $\mathrm{C}$ & -5.80846900 & 5.98691700 & 0.73089800 & $\mathrm{C}$ & -5.73036300 & -1.65655400 & -2.14279600 \\
\hline $\mathrm{H}$ & -6.67459600 & 5.44719200 & 0.33829200 & $\mathrm{H}$ & -6.10302100 & -1.51905000 & -1.12940200 \\
\hline $\mathrm{H}$ & -6.05517200 & 7.05244500 & 0.73347800 & $\mathrm{H}$ & -6.58605300 & -1.60180300 & -2.82522500 \\
\hline $\mathrm{H}$ & -5.65675700 & 5.68068500 & 1.76948400 & $\mathrm{H}$ & -5.06801800 & -0.82396500 & -2.36682200 \\
\hline $\mathrm{C}$ & 0.58640200 & 1.03645100 & -3.72299400 & $\mathrm{C}$ & -6.01513800 & -4.08253500 & -1.73330600 \\
\hline $\mathrm{C}$ & 0.91838900 & -0.30698300 & -4.40426800 & $\mathrm{H}$ & -5.71634400 & -5.10859500 & -1.93279700 \\
\hline $\mathrm{H}$ & 0.81723500 & -1.13619700 & -3.70161500 & $\mathrm{H}$ & -7.00309300 & -3.93611300 & -2.18476100 \\
\hline $\mathrm{H}$ & 1.95696900 & -0.28646700 & -4.75189500 & $\mathrm{H}$ & -6.13644300 & -3.95764500 & -0.65522000 \\
\hline $\mathrm{H}$ & 0.28485700 & -0.50901800 & -5.26922800 & $\mathrm{C}$ & -4.84411400 & -3.27921900 & -3.82740600 \\
\hline $\mathrm{C}$ & 0.84574100 & 2.19718900 & -4.70042200 & $\mathrm{H}$ & -4.18745100 & -2.52628200 & -4.27177100 \\
\hline
\end{tabular}




\begin{tabular}{|c|c|c|c|c|c|c|c|}
\hline $\mathrm{H}$ & -5.81128900 & -3.21603400 & -4.33750600 & $\mathrm{H}$ & 9.35634000 & -1.72923900 & -3.47110100 \\
\hline $\mathrm{H}$ & -4.42553000 & -4.26329700 & -4.04463200 & $\mathrm{H}$ & 7.61386000 & -1.47556000 & -3.67255100 \\
\hline \multirow{3}{*}{\multicolumn{4}{|c|}{$\left.\left[{ }^{\left({ }^{t \mathrm{Bu}}(\mathrm{PNP}) \mathrm{Fe}\right)}\right)_{2} \mathrm{Se}\right](9), 9 \mathrm{et}$}} & $\mathrm{C}$ & 3.18993900 & 5.85693000 & -0.79213100 \\
\hline & & & & $\mathrm{C}$ & 3.31517900 & 6.71609000 & 0.48802900 \\
\hline & & & & $\mathrm{H}$ & 2.50439900 & 6.51432000 & 1.19183900 \\
\hline $\mathrm{Se}$ & 0.00424000 & -1.89154000 & 0.01042900 & $\mathrm{H}$ & 3.28432900 & 7.78207000 & 0.23964900 \\
\hline $\mathrm{Fe}$ & 1.97284000 & -1.00060000 & 1.13319900 & $\mathrm{H}$ & 4.25970900 & 6.51292000 & 1.00014900 \\
\hline $\mathrm{P}$ & 1.37614000 & 0.72512000 & 3.04308900 & $\mathrm{C}$ & 1.85203900 & 6.18410000 & -1.49447100 \\
\hline $\mathrm{Fe}$ & -1.96631000 & -1.02531100 & -1.12983100 & $\mathrm{H}$ & 1.74400900 & 5.59871000 & -2.41191100 \\
\hline $\mathrm{P}$ & 3.50924000 & -3.00301000 & 1.27791900 & $\mathrm{H}$ & 1.80686900 & 7.24526000 & -1.76013100 \\
\hline $\mathrm{P}$ & -3.48806000 & -3.03914100 & -1.26768100 & $\mathrm{H}$ & 0.99515900 & 5.96512000 & -0.85347100 \\
\hline $\mathrm{N}$ & 3.44206000 & 0.30206000 & 0.65817900 & $\mathrm{C}$ & 4.33232900 & 6.25565000 & -1.74484100 \\
\hline $\mathrm{N}$ & -3.44555000 & 0.26941900 & -0.66575100 & $\mathrm{H}$ & 5.31404900 & 6.08911000 & -1.29362100 \\
\hline $\mathrm{C}$ & 4.67482000 & -0.00213000 & 0.09193900 & $\mathrm{H}$ & 4.25588900 & 7.31968000 & -1.98576100 \\
\hline $\mathrm{C}$ & 5.48222000 & -1.14574000 & 0.27552900 & $\mathrm{H}$ & 4.28909900 & 5.69875000 & -2.68485100 \\
\hline $\mathrm{C}$ & 6.68303000 & -1.19914000 & -0.42775100 & $\mathrm{C}$ & -0.34758000 & 0.59456000 & 3.90244900 \\
\hline $\mathrm{H}$ & 7.30839000 & -2.07362000 & -0.27864100 & $\mathrm{C}$ & -0.37103000 & -0.72889000 & 4.69509900 \\
\hline $\mathrm{C}$ & 7.15146000 & -0.16829000 & -1.27482100 & $\mathrm{H}$ & -0.20377000 & -1.58103000 & 4.03241900 \\
\hline $\mathrm{C}$ & 6.38691000 & 0.99079000 & -1.36331100 & $\mathrm{H}$ & -1.35670000 & -0.85449100 & 5.15610900 \\
\hline $\mathrm{H}$ & 6.72548000 & 1.83457000 & -1.95120100 & $\mathrm{H}$ & 0.37244000 & -0.76324000 & 5.49225900 \\
\hline $\mathrm{C}$ & 5.17295000 & 1.08527000 & -0.67307100 & $\mathrm{C}$ & -1.43082000 & 0.50261900 & 2.80824900 \\
\hline $\mathrm{C}$ & 4.23437000 & 2.16309000 & -0.47874100 & $\mathrm{H}$ & -1.58703000 & 1.45232900 & 2.29484900 \\
\hline $\mathrm{C}$ & 4.23150900 & 3.50346000 & -0.87668100 & $\mathrm{H}$ & -2.38132000 & 0.23672900 & 3.28284900 \\
\hline $\mathrm{H}$ & 5.02703900 & 3.86422000 & -1.51616100 & $\mathrm{H}$ & -1.20505000 & -0.26317100 & 2.06435900 \\
\hline $\mathrm{C}$ & 3.23528900 & 4.36233000 & -0.42158100 & $\mathrm{C}$ & -0.71492000 & 1.77170000 & 4.82360900 \\
\hline $\mathrm{C}$ & 2.25190900 & 3.82936000 & 0.44322900 & $\mathrm{H}$ & -0.07368000 & 1.85176000 & 5.70157900 \\
\hline $\mathrm{H}$ & 1.48414900 & 4.49204000 & 0.82937900 & $\mathrm{H}$ & -1.73984000 & 1.62632900 & 5.18281900 \\
\hline $\mathrm{C}$ & 2.20283000 & 2.49540000 & 0.84946900 & $\mathrm{H}$ & -0.69521100 & 2.72652000 & 4.29300900 \\
\hline $\mathrm{C}$ & 3.21233000 & 1.64134000 & 0.35858900 & $\mathrm{C}$ & 2.65698000 & 1.49845000 & 4.26768900 \\
\hline $\mathrm{C}$ & 1.06283000 & 2.05040000 & 1.74674900 & $\mathrm{C}$ & 2.45226900 & 3.01046000 & 4.47789900 \\
\hline $\mathrm{C}$ & 5.19033000 & -2.19034000 & 1.33307900 & $\mathrm{H}$ & 2.56490900 & 3.56305000 & 3.54353900 \\
\hline $\mathrm{H}$ & 5.22171000 & -1.69342000 & 2.30658900 & $\mathrm{H}$ & 3.22076900 & 3.37900000 & 5.16722900 \\
\hline $\mathrm{H}$ & 5.98165000 & -2.94271000 & 1.33717900 & $\mathrm{H}$ & 1.48265900 & 3.25493000 & 4.91004900 \\
\hline $\mathrm{C}$ & 8.49040000 & -0.34797900 & -2.01456100 & $\mathrm{C}$ & 4.07128000 & 1.28563000 & 3.68906900 \\
\hline $\mathrm{C}$ & 8.83903000 & 0.86909100 & -2.89089100 & $\mathrm{H}$ & 4.28748000 & 0.22923000 & 3.52527900 \\
\hline $\mathrm{H}$ & 8.07472000 & 1.05313000 & -3.65075100 & $\mathrm{H}$ & 4.80138000 & 1.67216000 & 4.40926900 \\
\hline $\mathrm{H}$ & 9.78573000 & 0.69108100 & -3.40876100 & $\mathrm{H}$ & 4.22037000 & 1.80889000 & 2.74637900 \\
\hline $\mathrm{H}$ & 8.95506000 & 1.77825100 & -2.29485100 & $\mathrm{C}$ & 2.60687000 & 0.76699000 & 5.62342900 \\
\hline $\mathrm{C}$ & 9.63540000 & -0.54265900 & -0.99325100 & $\mathrm{H}$ & 1.68118000 & 0.94041000 & 6.17123900 \\
\hline $\mathrm{H}$ & 9.71760000 & 0.32550100 & -0.33371100 & $\mathrm{H}$ & 3.42832000 & 1.13195000 & 6.24950900 \\
\hline $\mathrm{H}$ & 10.59225000 & -0.67116900 & -1.50975100 & $\mathrm{H}$ & 2.73982000 & -0.31173000 & 5.50988900 \\
\hline $\mathrm{H}$ & 9.47530000 & -1.42312900 & -0.36662100 & $\mathrm{C}$ & 3.43153000 & -4.01137000 & 2.91163900 \\
\hline $\mathrm{C}$ & 8.41238000 & -1.58915000 & -2.93418100 & $\mathrm{C}$ & 4.39633000 & -5.20612000 & 2.99711900 \\
\hline $\mathrm{H}$ & 8.21344000 & -2.50127000 & -2.36664100 & $\mathrm{H}$ & 4.14457000 & -5.99421000 & 2.28618900 \\
\hline
\end{tabular}




\begin{tabular}{|c|c|c|c|c|c|c|c|}
\hline $\mathrm{H}$ & 4.33747000 & -5.64409000 & 4.00011900 & $\mathrm{H}$ & -5.96017000 & -2.99384100 & -1.33647100 \\
\hline $\mathrm{H}$ & 5.43568000 & -4.91108000 & 2.83224900 & $\mathrm{C}$ & -8.47986000 & -0.34010100 & 2.04166900 \\
\hline $\mathrm{C}$ & 3.75580000 & -3.03666000 & 4.06296900 & $\mathrm{C}$ & -9.45420000 & 0.76735900 & 1.57656900 \\
\hline $\mathrm{H}$ & 4.81232000 & -2.76386000 & 4.09578900 & $\mathrm{H}$ & -9.04250000 & 1.76505900 & 1.74474900 \\
\hline $\mathrm{H}$ & 3.51468000 & -3.52069000 & 5.01510900 & $\mathrm{H}$ & -10.40093000 & 0.70001900 & 2.12271900 \\
\hline $\mathrm{H}$ & 3.16193000 & -2.12187000 & 3.99654900 & $\mathrm{H}$ & -9.66884000 & 0.67195900 & 0.50862900 \\
\hline $\mathrm{C}$ & 1.98008000 & -4.49546000 & 3.11103900 & $\mathrm{C}$ & -9.17373000 & -1.69912100 & 1.83380900 \\
\hline $\mathrm{H}$ & 1.27322000 & -3.66372000 & 3.07757900 & $\mathrm{H}$ & -9.44444000 & -1.86319100 & 0.78709900 \\
\hline $\mathrm{H}$ & 1.89435000 & -4.97478000 & 4.09258900 & $\mathrm{H}$ & -10.09581000 & -1.73390100 & 2.42071900 \\
\hline $\mathrm{H}$ & 1.67152000 & -5.22208000 & 2.36065900 & $\mathrm{H}$ & -8.54269000 & -2.53077100 & 2.15936900 \\
\hline $\mathrm{C}$ & 3.60341000 & -4.16094000 & -0.25355100 & $\mathrm{C}$ & -8.20789000 & -0.17405100 & 3.55499900 \\
\hline $\mathrm{C}$ & 3.39434000 & -3.26795000 & -1.49671100 & $\mathrm{H}$ & -7.53175000 & -0.95412100 & 3.91569900 \\
\hline $\mathrm{H}$ & 2.40671000 & -2.80465000 & -1.49215100 & $\mathrm{H}$ & -9.14199000 & -0.24240100 & 4.12236900 \\
\hline $\mathrm{H}$ & 3.47040000 & -3.89650000 & -2.39164100 & $\mathrm{H}$ & -7.75039000 & 0.79223900 & 3.77898900 \\
\hline $\mathrm{H}$ & 4.15145000 & -2.48639000 & -1.57596100 & $\mathrm{C}$ & -3.23947100 & 5.83482900 & 0.75343900 \\
\hline $\mathrm{C}$ & 4.95837000 & -4.87903000 & -0.39607100 & $\mathrm{C}$ & -3.36907100 & 6.68522900 & -0.53213100 \\
\hline $\mathrm{H}$ & 5.77689000 & -4.17408000 & -0.55062100 & $\mathrm{H}$ & -2.55532100 & 6.48580900 & -1.23315100 \\
\hline $\mathrm{H}$ & 4.91911000 & -5.52424000 & -1.28075100 & $\mathrm{H}$ & -3.34726100 & 7.75288900 & -0.29016100 \\
\hline $\mathrm{H}$ & 5.20207000 & -5.51093000 & 0.45779900 & $\mathrm{H}$ & -4.31093100 & 6.47141900 & -1.04483100 \\
\hline $\mathrm{C}$ & 2.46479000 & -5.19578000 & -0.21197100 & $\mathrm{C}$ & -4.38698100 & 6.23010900 & 1.70142900 \\
\hline $\mathrm{H}$ & 2.61270000 & -5.95034000 & 0.56328900 & $\mathrm{H}$ & -5.36639100 & 6.05246900 & 1.24937900 \\
\hline $\mathrm{H}$ & 2.43399000 & -5.72108000 & -1.17303100 & $\mathrm{H}$ & -4.31989100 & 7.29625900 & 1.93555900 \\
\hline $\mathrm{H}$ & 1.49406000 & -4.71698000 & -0.06560100 & $\mathrm{H}$ & -4.34092100 & 5.67973900 & 2.64515900 \\
\hline $\mathrm{C}$ & -4.67727000 & -0.04275100 & -0.10058100 & $\mathrm{C}$ & -1.90557100 & 6.17676900 & 1.45632900 \\
\hline $\mathrm{C}$ & -5.47421000 & -1.18989100 & -0.27988100 & $\mathrm{H}$ & -1.79452100 & 5.59756900 & 2.37732900 \\
\hline $\mathrm{C}$ & -6.67901000 & -1.25431100 & 0.42478900 & $\mathrm{H}$ & -1.86932100 & 7.23977900 & 1.71588900 \\
\hline $\mathrm{H}$ & -7.29056000 & -2.13568100 & 0.27581900 & $\mathrm{H}$ & -1.04581100 & 5.96092900 & 0.81812900 \\
\hline $\mathrm{C}$ & -7.15266000 & -0.22748100 & 1.26556900 & $P$ & -1.37914000 & 0.69358900 & -3.04871100 \\
\hline $\mathrm{C}$ & -6.39431000 & 0.94136900 & 1.34830900 & $\mathrm{C}$ & 0.34722000 & 0.57172000 & -3.90337100 \\
\hline $\mathrm{H}$ & -6.74693000 & 1.78323900 & 1.93473900 & $\mathrm{C}$ & 0.38268000 & -0.75517000 & -4.68980100 \\
\hline $\mathrm{C}$ & -5.18455000 & 1.04631900 & 0.66147900 & $\mathrm{H}$ & 0.21976000 & -1.60562000 & -4.02384100 \\
\hline $\mathrm{C}$ & -4.25332000 & 2.13095900 & 0.46116900 & $\mathrm{H}$ & 1.37071000 & -0.87553000 & -5.14715100 \\
\hline $\mathrm{C}$ & -4.26180100 & 3.47304900 & 0.85128900 & $\mathrm{H}$ & -0.35795000 & -0.79878000 & -5.48916100 \\
\hline $\mathrm{H}$ & -5.06076100 & 3.83108900 & 1.48800900 & $\mathrm{C}$ & 0.70811000 & 1.74729000 & -4.82900100 \\
\hline $\mathrm{C}$ & -3.27216100 & 4.33771900 & 0.39186900 & $\mathrm{H}$ & 0.06855000 & 1.81867000 & -5.70896100 \\
\hline $\mathrm{C}$ & -2.28408100 & 3.80736900 & -0.46860100 & $\mathrm{H}$ & 1.73502000 & 1.60807000 & -5.18495100 \\
\hline $\mathrm{H}$ & -1.52089100 & 4.47368900 & -0.85757100 & $\mathrm{H}$ & 0.67985900 & 2.70425000 & -4.30271100 \\
\hline $\mathrm{C}$ & -2.22424000 & 2.47144900 & -0.86750100 & $\mathrm{C}$ & 1.42839000 & 0.49279000 & -2.80612100 \\
\hline $\mathrm{C}$ & -3.22744000 & 1.61233900 & -0.37276100 & $\mathrm{H}$ & 1.57779000 & 1.44618000 & -2.29748100 \\
\hline $\mathrm{C}$ & -1.07948000 & 2.03011900 & -1.76060100 & $\mathrm{H}$ & 2.38164000 & 0.23014000 & -3.27694100 \\
\hline $\mathrm{H}$ & -0.29314000 & 1.59231000 & -1.13841100 & $\mathrm{H}$ & 1.20557000 & -0.27009000 & -2.05837100 \\
\hline $\mathrm{H}$ & -0.63459100 & 2.90843000 & -2.23423100 & $\mathrm{C}$ & -2.66317000 & 1.44924900 & -4.28121100 \\
\hline $\mathrm{C}$ & -5.17352000 & -2.23657100 & -1.33273100 & $\mathrm{C}$ & -2.47001100 & 2.96168900 & -4.49956100 \\
\hline $\mathrm{H}$ & -5.20399000 & -1.74447100 & -2.30877100 & $\mathrm{H}$ & -2.58607100 & 3.51847900 & -3.56812100 \\
\hline
\end{tabular}




\begin{tabular}{|c|c|c|c|c|c|c|c|}
\hline $\mathrm{H}$ & -3.24180100 & 3.32067900 & -5.19024100 & $\mathrm{Fe}$ & -1.95150000 & -1.00865000 & -1.16125000 \\
\hline $\mathrm{H}$ & -1.50266100 & 3.21125900 & -4.93390100 & $\mathrm{P}$ & 3.44800000 & -3.00196000 & 1.30556000 \\
\hline $\mathrm{C}$ & -4.07719000 & 1.22852900 & -3.70485100 & $\mathrm{P}$ & -3.43167000 & -3.03883000 & -1.28894000 \\
\hline $\mathrm{H}$ & -4.28628000 & 0.17124900 & -3.53756100 & $\mathrm{~N}$ & 3.43777000 & 0.29828000 & 0.67101000 \\
\hline $\mathrm{H}$ & -4.80840000 & 1.60690900 & -4.42826100 & $\mathrm{~N}$ & -3.44183000 & 0.26547000 & -0.68083000 \\
\hline $\mathrm{H}$ & -4.23239000 & 1.75433900 & -2.76458100 & $\mathrm{C}$ & 4.66169000 & -0.02707000 & 0.09940000 \\
\hline $\mathrm{C}$ & -2.60432000 & 0.71071900 & -5.63272100 & $\mathrm{C}$ & 5.45014000 & -1.18378000 & 0.28328000 \\
\hline $\mathrm{H}$ & -1.67857000 & 0.88780900 & -6.17925100 & $\mathrm{C}$ & 6.64596000 & -1.26053000 & -0.42612000 \\
\hline $\mathrm{H}$ & -3.42690000 & 1.06618900 & -6.26276100 & $\mathrm{H}$ & 7.25743000 & -2.14486000 & -0.27731000 \\
\hline $\mathrm{H}$ & -2.72982000 & -0.36830100 & -5.51353100 & $\mathrm{C}$ & 7.12686000 & -0.24080000 & -1.27998000 \\
\hline $\mathrm{C}$ & -3.39889000 & -4.05625100 & -2.89530100 & $\mathrm{C}$ & 6.38143000 & 0.93066000 & -1.36963000 \\
\hline $\mathrm{C}$ & -3.72759000 & -3.09042100 & -4.05296100 & $\mathrm{H}$ & 6.73122000 & 1.76564000 & -1.96347000 \\
\hline $\mathrm{H}$ & -4.78651000 & -2.82782100 & -4.09089100 & $\mathrm{C}$ & 5.17277000 & 1.04878000 & -0.67354000 \\
\hline $\mathrm{H}$ & -3.47841000 & -3.57707100 & -5.00169100 & $\mathrm{C}$ & 4.25086000 & 2.14136000 & -0.48045000 \\
\hline $\mathrm{H}$ & -3.14283000 & -2.16957100 & -3.98923100 & $\mathrm{C}$ & 4.26269000 & 3.47925000 & -0.88713000 \\
\hline $\mathrm{C}$ & -4.35488000 & -5.25833100 & -2.97697100 & $\mathrm{H}$ & 5.05992000 & 3.82649000 & -1.53192000 \\
\hline $\mathrm{H}$ & -4.09975000 & -6.04063100 & -2.26090100 & $\mathrm{C}$ & 3.27787000 & 4.35277000 & -0.43492000 \\
\hline $\mathrm{H}$ & -4.28972000 & -5.70147100 & -3.97732100 & $\mathrm{C}$ & 2.29024000 & 3.83732000 & 0.43569000 \\
\hline $\mathrm{H}$ & -5.39685000 & -4.96995100 & -2.81696100 & $\mathrm{H}$ & 1.53053000 & 4.51157000 & 0.81764000 \\
\hline $\mathrm{C}$ & -1.94354000 & -4.53123100 & -3.08827100 & $\mathrm{C}$ & 2.22666000 & 2.50700000 & 0.85102000 \\
\hline $\mathrm{H}$ & -1.24242000 & -3.69454100 & -3.05674100 & $\mathrm{C}$ & 3.22513000 & 1.63803000 & 0.36393000 \\
\hline $\mathrm{H}$ & -1.85158000 & -5.01477100 & -4.06719100 & $\mathrm{C}$ & 1.08030000 & 2.08001000 & 1.74905000 \\
\hline $\mathrm{H}$ & -1.63229000 & -5.25208100 & -2.33341100 & $\mathrm{C}$ & 5.14375000 & -2.21795000 & 1.34702000 \\
\hline $\mathrm{C}$ & -3.57996000 & -4.18940100 & 0.26978900 & $\mathrm{H}$ & 5.18861000 & -1.71619000 & 2.31751000 \\
\hline $\mathrm{C}$ & -3.37828000 & -3.28948100 & 1.50914900 & $\mathrm{H}$ & 5.92116000 & -2.98469000 & 1.35242000 \\
\hline $\mathrm{H}$ & -2.39321000 & -2.82074100 & 1.50464900 & $\mathrm{C}$ & 8.45859000 & -0.44574000 & -2.02625000 \\
\hline $\mathrm{H}$ & -3.45291000 & -3.91425100 & 2.40688900 & $\mathrm{C}$ & 8.82209000 & 0.76120000 & -2.91062000 \\
\hline $\mathrm{H}$ & -4.13971000 & -2.51158100 & 1.58264900 & $\mathrm{H}$ & 8.05667000 & 0.95368000 & -3.66726000 \\
\hline $\mathrm{C}$ & -4.93186000 & -4.91328100 & 0.41215900 & $\mathrm{H}$ & 9.76295000 & 0.56532000 & -3.43267000 \\
\hline $\mathrm{H}$ & -5.75409000 & -4.21172100 & 0.56248900 & $\mathrm{H}$ & 8.95592000 & 1.67144000 & -2.31999000 \\
\hline $\mathrm{H}$ & -4.89131000 & -5.55546100 & 1.29900900 & $\mathrm{C}$ & 9.60584000 & -0.65406000 & -1.01016000 \\
\hline $\mathrm{H}$ & -5.17081000 & -5.54906100 & -0.44016100 & $\mathrm{H}$ & 9.70542000 & 0.21570000 & -0.35512000 \\
\hline $\mathrm{C}$ & -2.43622000 & -5.21899100 & 0.23597900 & $\mathrm{H}$ & 10.55783000 & -0.80032000 & -1.53093000 \\
\hline $\mathrm{H}$ & -2.57842000 & -5.97821100 & -0.53584100 & $\mathrm{H}$ & 9.43476000 & -1.52893000 & -0.37861000 \\
\hline $\mathrm{H}$ & -2.40512000 & -5.73938100 & 1.19970900 & $\mathrm{C}$ & 8.35577000 & -1.68989000 & -2.93940000 \\
\hline $\mathrm{H}$ & -1.46738000 & -4.73637100 & 0.08954900 & $\mathrm{H}$ & 8.14543000 & -2.59605000 & -2.36645000 \\
\hline $\mathrm{H}$ & 0.27949000 & 1.60151000 & 1.12877900 & $\mathrm{H}$ & 9.29444000 & -1.84757000 & -3.48073000 \\
\hline $\mathrm{H}$ & 0.61119900 & 2.92809000 & 2.21507900 & $\mathrm{H}$ & 7.55521000 & -1.56713000 & -3.67410000 \\
\hline & & & & $\mathrm{C}$ & 3.24781000 & 5.84525000 & -0.81552000 \\
\hline \multirow{2}{*}{\multicolumn{4}{|c|}{$\left.\left[{ }^{(\mathrm{Bu}}(\mathrm{PNP}) \mathrm{Fe}\right)_{2} \mathrm{Se}\right](9)$, let }} & $\mathrm{C}$ & 3.38809000 & 6.71162000 & 0.45820000 \\
\hline & & & & $\mathrm{H}$ & 2.57775000 & 6.52438000 & 1.16659000 \\
\hline se & 0.00000000 & -1.76824000 & 0.01779000 & $\mathrm{H}$ & 3.36896000 & 7.77617000 & 0.20258000 \\
\hline $\mathrm{Fe}$ & 1.95614000 & -0.98052000 & 1.16811000 & $\mathrm{H}$ & 4.33209000 & 6.50071000 & 0.96815000 \\
\hline $\mathrm{P}$ & 1.37283000 & 0.75981000 & 3.05537000 & $\mathrm{C}$ & 1.91066000 & 6.18282000 & -1.51456000 \\
\hline
\end{tabular}




\begin{tabular}{|c|c|c|c|c|c|c|c|}
\hline $\mathrm{H}$ & 1.79263000 & 5.59282000 & -2.42782000 & $\mathrm{H}$ & 1.82393000 & -4.93840000 & 4.14051000 \\
\hline $\mathrm{H}$ & 1.87625000 & 7.24271000 & -1.78692000 & $\mathrm{H}$ & 1.58089000 & -5.18210000 & 2.41084000 \\
\hline $\mathrm{H}$ & 1.05370000 & 5.97744000 & -0.86905000 & $\mathrm{C}$ & 3.51766000 & -4.17152000 & -0.21968000 \\
\hline $\mathrm{C}$ & 4.39058000 & 6.22457000 & -1.77566000 & $\mathrm{C}$ & 3.32102000 & -3.28458000 & -1.46902000 \\
\hline $\mathrm{H}$ & 5.37225000 & 6.05056000 & -1.32713000 & $\mathrm{H}$ & 2.34187000 & -2.80413000 & -1.46652000 \\
\hline $\mathrm{H}$ & 4.32486000 & 7.28757000 & -2.02418000 & $\mathrm{H}$ & 3.38565000 & -3.92096000 & -2.35931000 \\
\hline $\mathrm{H}$ & 4.33750000 & 5.66124000 & -2.71134000 & $\mathrm{H}$ & 4.09071000 & -2.51623000 & -1.55485000 \\
\hline $\mathrm{C}$ & -0.35283000 & 0.66050000 & 3.91518000 & $\mathrm{C}$ & 4.86014000 & -4.91279000 & -0.36189000 \\
\hline $\mathrm{C}$ & -0.39793000 & -0.65977000 & 4.71199000 & $\mathrm{H}$ & 5.68912000 & -4.22199000 & -0.52424000 \\
\hline $\mathrm{H}$ & -0.24179000 & -1.51645000 & 4.05239000 & $\mathrm{H}$ & 4.80744000 & -5.56333000 & -1.24196000 \\
\hline $\mathrm{H}$ & -1.38626000 & -0.76911000 & 5.17142000 & $\mathrm{H}$ & 5.09713000 & -5.54234000 & 0.49536000 \\
\hline $\mathrm{H}$ & 0.34350000 & -0.70289000 & 5.51072000 & $\mathrm{C}$ & 2.36226000 & -5.18734000 & -0.16822000 \\
\hline $\mathrm{C}$ & -1.43930000 & 0.58484000 & 2.82301000 & $\mathrm{H}$ & 2.50007000 & -5.94057000 & 0.61022000 \\
\hline $\mathrm{H}$ & -1.57784000 & 1.53500000 & 2.30533000 & $\mathrm{H}$ & 2.31843000 & -5.71687000 & -1.12643000 \\
\hline $\mathrm{H}$ & -2.39412000 & 0.33944000 & 3.29987000 & $\mathrm{H}$ & 1.39986000 & -4.69238000 & -0.01987000 \\
\hline $\mathrm{H}$ & -1.23016000 & -0.18758000 & 2.08162000 & $\mathrm{C}$ & -4.66599000 & -0.06440000 & -0.11139000 \\
\hline $\mathrm{C}$ & -0.69928000 & 1.84650000 & 4.83311000 & $\mathrm{C}$ & -5.44594000 & -1.22325000 & -0.29004000 \\
\hline $\mathrm{H}$ & -0.05604000 & 1.91848000 & 5.71022000 & $\mathrm{C}$ & -6.64710000 & -1.30710000 & 0.41866000 \\
\hline $\mathrm{H}$ & -1.72618000 & 1.71974000 & 5.19361000 & $\mathrm{H}$ & -7.24634000 & -2.19704000 & 0.27040000 \\
\hline $\mathrm{H}$ & -0.66353000 & 2.79905000 & 4.29936000 & $\mathrm{C}$ & -7.13238000 & -0.28879000 & 1.26353000 \\
\hline $\mathrm{C}$ & 2.66513000 & 1.51990000 & 4.27586000 & $\mathrm{C}$ & -6.39059000 & 0.89067000 & 1.34706000 \\
\hline $\mathrm{C}$ & 2.48179000 & 3.03545000 & 4.48115000 & $\mathrm{H}$ & -6.75324000 & 1.72522000 & 1.93778000 \\
\hline $\mathrm{H}$ & 2.59474000 & 3.58284000 & 3.54375000 & $\mathrm{C}$ & -5.18492000 & 1.01535000 & 0.65622000 \\
\hline $\mathrm{H}$ & 3.26040000 & 3.39637000 & 5.16311000 & $\mathrm{C}$ & -4.26766000 & 2.11229000 & 0.45710000 \\
\hline $\mathrm{H}$ & 1.51904000 & 3.29492000 & 4.91989000 & $\mathrm{C}$ & -4.28806000 & 3.45226000 & 0.85461000 \\
\hline $\mathrm{C}$ & 4.07600000 & 1.28623000 & 3.69672000 & $\mathrm{H}$ & -5.08859000 & 3.79939000 & 1.49538000 \\
\hline $\mathrm{H}$ & 4.27964000 & 0.22636000 & 3.53940000 & $\mathrm{C}$ & -3.30708000 & 4.32862000 & 0.39854000 \\
\hline $\mathrm{H}$ & 4.81147000 & 1.66832000 & 4.41382000 & $\mathrm{C}$ & -2.31511000 & 3.81255000 & -0.46619000 \\
\hline $\mathrm{H}$ & 4.23046000 & 1.80152000 & 2.75055000 & $\mathrm{H}$ & -1.55794000 & 4.48816000 & -0.85081000 \\
\hline $\mathrm{C}$ & 2.60677000 & 0.79346000 & 5.63394000 & $\mathrm{C}$ & -2.24348000 & 2.47972000 & -0.87269000 \\
\hline $\mathrm{H}$ & 1.68462000 & 0.98158000 & 6.18287000 & $\mathrm{C}$ & -3.23820000 & 1.60867000 & -0.38187000 \\
\hline $\mathrm{H}$ & 3.43443000 & 1.14837000 & 6.25760000 & $\mathrm{C}$ & -1.09228000 & 2.05280000 & -1.76469000 \\
\hline $\mathrm{H}$ & 2.72407000 & -0.28737000 & 5.52324000 & $\mathrm{H}$ & -0.30543000 & 1.61762000 & -1.14122000 \\
\hline $\mathrm{C}$ & 3.36722000 & -4.00274000 & 2.94484000 & $\mathrm{H}$ & -0.65299000 & 2.93715000 & -2.23243000 \\
\hline $\mathrm{C}$ & 4.31155000 & -5.21379000 & 3.02939000 & $\mathrm{C}$ & -5.13039000 & -2.26231000 & -1.34618000 \\
\hline $\mathrm{H}$ & 4.04000000 & -6.00166000 & 2.32556000 & $\mathrm{H}$ & -5.17122000 & -1.76756000 & -2.32050000 \\
\hline $\mathrm{H}$ & 4.25333000 & -5.64470000 & 4.03549000 & $\mathrm{H}$ & -5.90431000 & -3.03257000 & -1.35117000 \\
\hline $\mathrm{H}$ & 5.35462000 & -4.93849000 & 2.85431000 & $\mathrm{C}$ & -8.45477000 & -0.42176000 & 2.04470000 \\
\hline $\mathrm{C}$ & 3.71671000 & -3.02908000 & 4.08964000 & $\mathrm{C}$ & -9.44550000 & 0.67463000 & 1.58806000 \\
\hline $\mathrm{H}$ & 4.77787000 & -2.77406000 & 4.11323000 & $\mathrm{H}$ & -9.04645000 & 1.67701000 & 1.75895000 \\
\hline $\mathrm{H}$ & 3.47478000 & -3.50516000 & 5.04561000 & $\mathrm{H}$ & -10.38916000 & 0.59232000 & 2.13749000 \\
\hline $\mathrm{H}$ & 3.13774000 & -2.10472000 & 4.02409000 & $\mathrm{H}$ & -9.66289000 & 0.58096000 & 0.52053000 \\
\hline $\mathrm{C}$ & 1.90941000 & -4.46094000 & 3.15804000 & $\mathrm{C}$ & -9.13192000 & -1.78891000 & 1.83472000 \\
\hline $\mathrm{H}$ & 1.21791000 & -3.61614000 & 3.13124000 & $\mathrm{H}$ & -9.40441000 & -1.95301000 & 0.78847000 \\
\hline
\end{tabular}




\begin{tabular}{|c|c|c|c|c|c|c|c|}
\hline $\mathrm{H}$ & -10.05123000 & -1.83743000 & 2.42501000 & $\mathrm{H}$ & -1.52842000 & 3.24179000 & -4.94415000 \\
\hline $\mathrm{H}$ & -8.48906000 & -2.61347000 & 2.15519000 & $\mathrm{C}$ & -4.07656000 & 1.22420000 & -3.71708000 \\
\hline $\mathrm{C}$ & -8.17873000 & -0.25785000 & 3.55758000 & $\mathrm{H}$ & -4.27461000 & 0.16383000 & -3.55601000 \\
\hline $\mathrm{H}$ & -7.49160000 & -1.03084000 & 3.91273000 & $\mathrm{H}$ & -4.81143000 & 1.59865000 & -4.43882000 \\
\hline $\mathrm{H}$ & -9.10956000 & -0.33963000 & 4.12857000 & $\mathrm{H}$ & -4.23799000 & 1.74271000 & -2.77387000 \\
\hline $\mathrm{H}$ & -7.73215000 & 0.71318000 & 3.78315000 & $\mathrm{C}$ & -2.59685000 & 0.72959000 & -5.64543000 \\
\hline $\mathrm{C}$ & -3.28601000 & 5.82368000 & 0.76933000 & $\mathrm{H}$ & -1.67344000 & 0.91952000 & -6.19161000 \\
\hline $\mathrm{C}$ & -3.42680000 & 6.68100000 & -0.51044000 & $\mathrm{H}$ & -3.42384000 & 1.07649000 & -6.27445000 \\
\hline $\mathrm{H}$ & -2.61341000 & 6.49320000 & -1.21517000 & $\mathrm{H}$ & -2.70898000 & -0.35121000 & -5.52902000 \\
\hline $\mathrm{H}$ & -3.41369000 & 7.74727000 & -0.26177000 & $\mathrm{C}$ & -3.33568000 & -4.05123000 & -2.92004000 \\
\hline $\mathrm{H}$ & -4.36821000 & 6.46208000 & -1.02179000 & $\mathrm{C}$ & -3.68429000 & -3.08786000 & -4.07392000 \\
\hline $\mathrm{C}$ & -4.43377000 & 6.20327000 & 1.72338000 & $\mathrm{H}$ & -4.74731000 & -2.84171000 & -4.10648000 \\
\hline $\mathrm{H}$ & -5.41308000 & 6.02062000 & 1.27310000 & $\mathrm{H}$ & -3.43182000 & -3.56832000 & -5.02496000 \\
\hline $\mathrm{H}$ & -4.37477000 & 7.26834000 & 1.96456000 & $\mathrm{H}$ & -3.11363000 & -2.15817000 & -4.01068000 \\
\hline $\mathrm{H}$ & -4.38036000 & 5.64691000 & 2.66321000 & $\mathrm{C}$ & -4.27274000 & -5.26811000 & -3.00201000 \\
\hline $\mathrm{C}$ & -1.95274000 & 6.17254000 & 1.47025000 & $\mathrm{H}$ & -4.00176000 & -6.04933000 & -2.29061000 \\
\hline $\mathrm{H}$ & -1.83424000 & 5.58857000 & 2.38731000 & $\mathrm{H}$ & -4.20534000 & -5.70607000 & -4.00449000 \\
\hline $\mathrm{H}$ & -1.92465000 & 7.23420000 & 1.73635000 & $\mathrm{H}$ & -5.31848000 & -4.99739000 & -2.83597000 \\
\hline $\mathrm{H}$ & -1.09287000 & 5.96779000 & 0.82840000 & $\mathrm{C}$ & -1.87399000 & -4.50294000 & -3.12088000 \\
\hline$P$ & -1.37236000 & 0.72002000 & -3.06108000 & $\mathrm{H}$ & -1.18645000 & -3.65494000 & -3.09063000 \\
\hline $\mathrm{C}$ & 0.35636000 & 0.62484000 & -3.91452000 & $\mathrm{H}$ & -1.77847000 & -4.98274000 & -4.10128000 \\
\hline $\mathrm{C}$ & 0.41109000 & -0.69848000 & -4.70573000 & $\mathrm{H}$ & -1.54817000 & -5.22062000 & -2.36911000 \\
\hline $\mathrm{H}$ & 0.25628000 & -1.55357000 & -4.04374000 & $\mathrm{C}$ & -3.50469000 & -4.19783000 & 0.24416000 \\
\hline $\mathrm{H}$ & 1.40202000 & -0.80476000 & -5.16024000 & $\mathrm{C}$ & -3.31639000 & -3.30227000 & 1.48856000 \\
\hline $\mathrm{H}$ & -0.32637000 & -0.74852000 & -5.50768000 & $\mathrm{H}$ & -2.33934000 & -2.81752000 & 1.48692000 \\
\hline $\mathrm{C}$ & 0.70047000 & 1.80858000 & -4.83620000 & $\mathrm{H}$ & -3.38197000 & -3.93320000 & 2.38269000 \\
\hline $\mathrm{H}$ & 0.06031000 & 1.87355000 & -5.71614000 & $\mathrm{H}$ & -4.08950000 & -2.53645000 & 1.56595000 \\
\hline $\mathrm{H}$ & 1.72943000 & 1.68561000 & -5.19211000 & $\mathrm{C}$ & -4.84584000 & -4.94192000 & 0.38444000 \\
\hline $\mathrm{H}$ & 0.65776000 & 2.76317000 & -4.30667000 & $\mathrm{H}$ & -5.67736000 & -4.25270000 & 0.54042000 \\
\hline $\mathrm{C}$ & 1.43903000 & 0.55882000 & -2.81798000 & $\mathrm{H}$ & -4.79484000 & -5.58872000 & 1.26739000 \\
\hline $\mathrm{H}$ & 1.57283000 & 1.51210000 & -2.30481000 & $\mathrm{H}$ & -5.07772000 & -5.57560000 & -0.47118000 \\
\hline $\mathrm{H}$ & 2.39642000 & 0.31396000 & -3.28989000 & $\mathrm{C}$ & -2.34650000 & -5.21110000 & 0.20439000 \\
\hline $\mathrm{H}$ & 1.22963000 & -0.20992000 & -2.07285000 & $\mathrm{H}$ & -2.47915000 & -5.96995000 & -0.56951000 \\
\hline $\mathrm{C}$ & -2.66487000 & 1.46352000 & -4.29187000 & $\mathrm{H}$ & -2.30537000 & -5.73410000 & 1.16631000 \\
\hline $\mathrm{C}$ & -2.49038000 & 2.97901000 & -4.50562000 & $\mathrm{H}$ & -1.38448000 & -4.71527000 & 0.05665000 \\
\hline $\mathrm{H}$ & -2.60834000 & 3.53121000 & -3.57167000 & $\mathrm{H}$ & 0.29489000 & 1.63416000 & 1.13145000 \\
\hline $\mathrm{H}$ & -3.26986000 & 3.33114000 & -5.19115000 & $\mathrm{H}$ & 0.63627000 & 2.96526000 & 2.21054000 \\
\hline
\end{tabular}




\section{S7 CRYstalographic Data}

\section{S7.1 X-Ray Crystal STRUCTURE Determinations}

Crystal data and details of the structure determinations are compiled in Tables S7-S9. Full shells of intensity data were collected at low temperature with a Bruker AXS Smart 1000 CCD diffractometer (Mo- $K_{\alpha}$ radiation, sealed X-ray tube, graphite monochromator; compound $\mathbf{3}$ ) or an Agilent Technologies Supernova-E CCD diffractometer (Mo- or $\mathrm{Cu}-K_{\alpha}$ radiation, microfocus Xray tubes, multilayer mirror optics; all other compounds). Detector frames (typically $\omega$-, occasionally $\varphi$-scans, scan width $0.4 \ldots 1^{\circ}$ ) were integrated by profile fitting. ${ }^{17-19}$ Data were corrected for air and detector absorption, Lorentz and polarization effects ${ }^{18,19}$ and scaled essentially by application of appropriate spherical harmonic functions. ${ }^{20-22}$ Absorption by the crystal was treated with a semiempirical multiscan method (as part of the scaling procedure) and augmented by a spherical correction, ${ }^{20-22}$ or numerically (Gaussian grid) ${ }^{22,23}$ For datasets collected with the microfocus tubes an illumination correction was performed as part of the numerical absorption correction..$^{22}$

The structures were solved by the charge flip procedure,,${ }^{24}$ by the heavy atom method combined with structure expansion by direct methods applied to difference structure factors ${ }^{25}$ or by ab initio dual space methods involving difference Fourier syntheses (VLD procedure) ${ }^{26}$ and refined by fullmatrix least squares methods based on $F^{2}$ against all unique reflections. ${ }^{27}$ All non-hydrogen atoms were given anisotropic displacement parameters. Hydrogen atoms were generally input at calculated positions and refined with a riding model. ${ }^{28}$ The positions of some hydrogen atoms in $\mathbf{4}$ - $0.5 n$-hexane were taken from a difference Fourier synthesis. These hydrogen atoms (H40 and H90) were then fully refined. Split atom models were used to refine disordered groups and/or solvent molecules. When found necessary, suitable geometry and adp restraints or constraints were applied. ${ }^{28,29}$ Due to severe disorder, electron density attributed to solvent of crystallization was removed from the structures of $4 \cdot 0.5 n$-hexane ( $n$-hexane), $8 \cdot 2 n$-pentane $\cdot 0.5 \mathrm{SPMe}_{3}(n$-pentane) and $9 \cdot 0.5 n$-hexane ( $n$-hexane) with the BYPASS procedure, ${ }^{30}$ as implemented in PLATON (squeeze/hybrid) ${ }^{31}$ Partial structure factors from the solvent masks were included in the refinement as separate contributions to $F_{\text {calc. }}$.

The final difference Fourier map of $9 \cdot 0.5 n$-hexane, calculated with the contributions to $F_{\text {calc }}$ from the solvent mask, showed a maximum (approx. $3.65 \mathrm{e} \cdot \AA^{-3}$ ) $2.07 \AA$ from P3. This feature is attributed to be due to the presence of a minor amount (ca. 8\%) of a complex where P3 has been oxidized by Se to give a ${ }^{t} \mathrm{Bu} 2 \mathrm{P}=\mathrm{Se}$ moiety $(\mathbf{9 a})$ in the crystal. This complex has been included in the final model.

In several attempts, compound $\left[{ }^{[\mathrm{Bu}}(\mathrm{PNP}) \mathrm{Fe}(\mathrm{CO})_{2}\right]$ (3) was found to co-crystallize in an isomorphous way with a small amount $(4-6 \%)$ of the monocarbonyl complex $\left[{ }^{i \mathrm{Bu}}(\mathrm{PNP}) \mathrm{Fe}(\mathrm{CO})\right]$ (6), as evident by a second position of the $\mathrm{Fe}$ atom associated with a corresponding reduction of 
the population of the ("axial") carbonyl ligand (C41 O41). All other atoms including the "equatorial" carbonyl ligand (C40 O40) essentially occupy the same positions in both complexes (Figure S33). Conversely, in the structure of $\mathbf{6}$ weak residual density peaks for a second $\mathrm{Fe}(\mathrm{CO})$ group also indicate the presence of a minute amount of $\mathbf{3}$.

CCDC 1967923 - 1967924, 2050707 - 2050708 and 2051083 - 2051087 contains the supplementary crystallographic data for this paper. These data can be obtained free of charge from the Cambridge Crystallographic Data Centre's and FIZ Karlsruhe's joint Access Service via https://www.ccdc.cam.ac.uk/structures/?. 


\section{S7.2 STRUCTURE PARAMETERS}

Table S7. Crystallographic data for complexes $2 \cdot n$-pentane, 3 and $4 \cdot 0.5 n$-hexane.

\begin{tabular}{|c|c|c|c|}
\hline & $\begin{array}{l}{\left[{ }^{[\mathrm{Bu}}(\mathrm{PNP}) \mathrm{Fe}(\mathrm{TEMPO})\right]} \\
(\mathbf{2}) \cdot n \text {-pentane }\end{array}$ & $\begin{array}{l}{\left[^{[\mathrm{Bu}}(\mathrm{PNP}) \mathrm{Fe}(\mathrm{CO})_{2}\right]_{0.96} \cdot} \\
\left.{ }^{[\mathrm{Bu}}(\mathrm{PNP}) \mathrm{Fe}(\mathrm{CO})\right]_{0.04} \\
\left([3]_{0.96} \cdot[\mathbf{6}]_{0.04}\right)\end{array}$ & $\begin{array}{l}\left.{ }^{[\mathrm{Bu}}(\mathrm{PNP}) \mathrm{Fe}(\mathrm{HCCPh})\right] \\
(\mathbf{4}) \cdot 0.5 n \text {-hexane }\end{array}$ \\
\hline formula & $\mathrm{C}_{52} \mathrm{H}_{92} \mathrm{FeN}_{2} \mathrm{OP}_{2}$ & $\mathrm{C}_{39.96} \mathrm{H}_{62} \mathrm{FeNO}_{1.96} \mathrm{P}_{2}$ & $\mathrm{C}_{49} \mathrm{H}_{75} \mathrm{FeNP}_{2}$ \\
\hline crystal system & triclinic & orthorhombic & triclinic \\
\hline space group & $P-1$ & $P b c a$ & $P-1$ \\
\hline$a / \AA$ & $11.5121(3)$ & $23.571(10)^{b}$ & $14.9109(3)$ \\
\hline$b / \AA$ & $12.9446(3)$ & $12.071(5)^{\mathrm{b}}$ & $15.2842(3)$ \\
\hline$c / \AA$ & $18.9389(5)$ & $27.200(13)^{b}$ & $23.1396(4)$ \\
\hline$\alpha /^{\circ}$ & $71.295(3)$ & & $88.7490(14)$ \\
\hline$\beta 1^{\circ}$ & $78.879(2)$ & & $71.4412(16)$ \\
\hline$\gamma /{ }^{\circ}$ & $80.800(2)$ & & $70.2810(16)$ \\
\hline$V / \AA^{3}$ & $2608.37(13)$ & $7739(6)^{b}$ & $4684.46(16)$ \\
\hline$Z$ & 2 & 8 & 4 \\
\hline$M_{\mathrm{r}}$ & 879.06 & 705.64 & 795.89 \\
\hline$F_{000}$ & 964 & 3044 & 1728 \\
\hline$d_{\mathrm{c}} / \mathrm{Mg} \cdot \mathrm{m}^{-3}$ & 1.119 & 1.211 & 1.128 \\
\hline$\mu / \mathrm{mm}^{-1}$ & 3.115 & 0.506 & 3.449 \\
\hline max., min. transmission factors & $1.000,0.549^{\mathrm{a}}$ & $0.746,0.673^{\mathrm{c}}$ & $1.000,0.629^{\mathrm{a}}$ \\
\hline $\mathrm{X}$-radiation, $\lambda / \AA$ & $\mathrm{Cu}-K_{\alpha}, 1.54184$ & Мо- $K_{\alpha}, 0.71073$ & $\mathrm{Cu}-K_{\alpha}, 1.54184$ \\
\hline data collect. temperat. /K & $120(1)$ & $100(1)$ & $120(1)$ \\
\hline$\theta$ range $1^{\circ}$ & 2.5 to 70.9 & 1.5 to 32.6 & 3.1 to 71.2 \\
\hline index ranges $h, k, l$ & $\pm 14,-15 \ldots 12, \pm 23$ & $\pm 35,-17 \ldots 18, \pm 39$ & $-18 \ldots 16, \pm 18, \pm 28$ \\
\hline \multirow{3}{*}{$\begin{array}{l}\text { reflections measured } \\
\qquad \begin{array}{l}\text { unique }\left[R_{\text {int }}\right] \\
\text { observed }[I \geq 2 \sigma(I)]\end{array}\end{array}$} & 66504 & 189969 & 128988 \\
\hline & $9943[0.057]$ & $13519[0.052]$ & $17944[0.059]$ \\
\hline & 8205 & 11123 & 14733 \\
\hline Phasing & charge flip & vld & vld \\
\hline data / restraints /parameters & 9943 / 48 / 547 & $13519 / 0 / 437$ & $17944 / 0$ / 945 \\
\hline GooF on $F^{2}$ & 1.020 & 1.059 & 1.025 \\
\hline$R$ indices $[F>4 \sigma(F)] R(F), w R\left(F^{2}\right)$ & $0.0525,0.1304$ & $0.0376,0.0861$ & $0.0382,0.0870$ \\
\hline$R$ indices (all data) $R(F), w R\left(F^{2}\right)$ & $0.0662,0.1400$ & $0.0529,0.0935$ & $0.0523,0.0932$ \\
\hline largest residual peaks $/ \mathrm{e} \cdot \AA^{-3}$ & $1.083,-0.316$ & $0.708,-0.271$ & $0.714,-0.328$ \\
\hline CCDC deposition number & 2051083 & 2050707 & 2050708 \\
\hline
\end{tabular}

${ }^{a}$ numerical absorption correction; ${ }^{b}$ standard uncertainties include systematic error contributions from Monte Carlo simulations;

${ }^{\mathrm{c}}$ semi-empirical absorption correction. 
Table S8. Crystallographic data for complexes $5 \cdot n$-hexane, 6 and $7 \cdot n$-hexane.

\begin{tabular}{|c|c|c|c|}
\hline & $\begin{array}{l}{ }_{t \mathrm{Bu}}(\mathrm{PNP}) \mathrm{Fe}\left(\mathrm{N}_{2} \mathrm{CPh}_{2}\right) \\
(\mathbf{5}) \cdot n \text {-hexane }\end{array}$ & $\begin{array}{l}{ }_{t \mathrm{Bu}}(\mathrm{PNP}) \mathrm{Fe}(\mathrm{CO}) \\
(\mathbf{6})\end{array}$ & $\begin{array}{l}\left({ }^{t \mathrm{Bu}}(\mathrm{PNP}) \mathrm{Fe}\right)_{2}\left(\mathrm{CO}_{3}\right) \\
(7) \cdot n \text {-hexane }\end{array}$ \\
\hline $\begin{array}{l}\text { formula } \\
\text { crystal system }\end{array}$ & $\begin{array}{l}\mathrm{C}_{57} \mathrm{H}_{86} \mathrm{FeN}_{3} \mathrm{P}_{2} \\
\text { monoclinic }\end{array}$ & $\begin{array}{l}\mathrm{C}_{39} \mathrm{H}_{62} \mathrm{FeNOP}_{2} \\
\text { orthorhombic }\end{array}$ & $\begin{array}{l}\mathrm{C}_{83} \mathrm{H}_{138} \mathrm{Fe}_{2} \mathrm{~N}_{2} \mathrm{O}_{3} \mathrm{P}_{4} \\
\text { monoclinic }\end{array}$ \\
\hline space group & $P 2{ }_{1} / n$ & $P b c a$ & $P 2{ }_{1} / c$ \\
\hline$a / \AA ̊$ & $16.7834(2)$ & $23.5272(2)$ & $15.7470(5)$ \\
\hline$b / \AA$ & $16.3422(2)$ & $12.10640(13)$ & $41.6751(12)$ \\
\hline$c / \AA$ & $19.9541(2)$ & $26.9186(3)$ & $13.8044(5)$ \\
\hline \multicolumn{4}{|l|}{$\alpha /^{\circ}$} \\
\hline$\beta /^{\circ}$ & $96.4451(11)$ & & $111.990(4)$ \\
\hline$V / \AA^{3}$ & $5438.37(12)$ & 7667.21(15) & $8400.2(5)$ \\
\hline$Z$ & 4 & 8 & 4 \\
\hline$M_{\mathrm{r}}$ & 931.07 & 678.68 & 1447.53 \\
\hline$F_{000}$ & 2020 & 2936 & 3144 \\
\hline$d_{\mathrm{c}} / \mathrm{Mg} \cdot \mathrm{m}^{-3}$ & 1.137 & 1.176 & 1.145 \\
\hline$\mu / \mathrm{mm}^{-1}$ & 3.049 & 4.151 & 3.825 \\
\hline max., min. transmission factors & $1.000,0.557^{\mathrm{a}}$ & $1.000,0.741^{\mathrm{b}}$ & $1.000,0.541^{\mathrm{a}}$ \\
\hline X-radiation, $\lambda / \AA$ & $\mathrm{Cu}-K_{\alpha}, 1.54184$ & $\mathrm{Cu}-K_{\alpha}, 1.54184$ & $\mathrm{Cu}-K_{\alpha}, 1.54184$ \\
\hline data collect. temperat. /K & $120(1)$ & $120(1)$ & $120(1)$ \\
\hline$\theta$ range $1^{\circ}$ & 3.3 to 71.6 & 3.3 to 71.0 & 3.0 to 71.4 \\
\hline index ranges $h, k, l$ & $\pm 20,-20 \ldots 18, \pm 24$ & $\pm 28, \pm 14,-32 \ldots 28$ & $-18 \ldots 19, \pm 51, \pm 16$ \\
\hline reflections measured & 243720 & 200041 & 202764 \\
\hline unique $\left[R_{\text {int }}\right]$ & $10486[0.078]$ & $7382[0.080]$ & $16168[0.180]$ \\
\hline observed $[I \geq 2 \sigma(I)]$ & 8411 & 6142 & 10423 \\
\hline phasing & heavy atom & charge flip & vld \\
\hline data / restraints /parameters & $10486 / 145$ / 586 & $7382 / 36 / 415$ & $16168 / 124$ / 885 \\
\hline GooF on $F^{2}$ & 1.026 & 1.027 & 1.055 \\
\hline$R$ indices $[F>4 \sigma(F)] R(F), w R\left(F^{2}\right)$ & $0.0532,0.1261$ & $0.0571,0.1459$ & $0.0810,0.1816$ \\
\hline$R$ indices (all data) $R(F), w R\left(F^{2}\right)$ & $0.0709,0.1359$ & $0.0710,0.1560$ & $0.1302,0.2051$ \\
\hline largest residual peaks $/ \mathrm{e} \cdot \AA^{-3}$ & $0.979,-0.578$ & $1.300,-0.830$ & $1.294,-0.566$ \\
\hline CCDC deposition number & 2051084 & 2051085 & 2051086 \\
\hline
\end{tabular}

${ }^{\mathrm{a}}$ numerical absorption correction; ${ }^{\mathrm{b}}$ semi-empirical absorption correction. 
Table S9. Crystallographic data for complexes 8 $2 n$-pentane $\cdot 0.5 \mathrm{SPMe}_{3}, 9 \cdot 0.5 n$-hexane and ${ }^{t \mathrm{Bu}}(\mathrm{PNP}) \mathrm{Fe}(\mathrm{CCPh})$.

\begin{tabular}{|c|c|c|c|}
\hline & $\begin{array}{l}\left({ }^{t \mathrm{Bu}}(\mathrm{PNP}) \mathrm{Fe}\right)_{2} \mathrm{~S} \\
(8) \cdot 1.75 n \text {-pentane } \\
\cdot 0.25 \mathrm{SPMe}_{3}\end{array}$ & $\begin{array}{l}\left.\left[{ }^{t \mathrm{tBu}}(\mathrm{PNP}) \mathrm{Fe}\right)_{2} \mathrm{Se}\right]_{0.92} \\
\left.\left[{ }^{\mathrm{tBu}}(\mathrm{PNP}) \mathrm{Fe}\right)_{2} \mathrm{Se}_{2}\right]_{0.08} \\
\left([\mathbf{9}]_{0.92}[\mathbf{9 a}]_{0.08}\right) \cdot 0.5 n- \\
\text { hexane }\end{array}$ & ${ }^{t \mathrm{Bu}}(\mathrm{PNP}) \mathrm{Fe}(\mathrm{CCPh})$ \\
\hline formula & $\mathrm{C}_{85.5} \mathrm{H}_{147.25} \mathrm{Fe}_{2} \mathrm{~N}_{2} \mathrm{P}_{4.15} \mathrm{~S}_{1.25}$ & $\mathrm{C}_{79} \mathrm{H}_{131} \mathrm{Fe}_{2} \mathrm{~N}_{2} \mathrm{P}_{4} \mathrm{Se}_{1.08}$ & $\mathrm{C}_{46} \mathrm{H}_{67} \mathrm{FeNP}_{2}$ \\
\hline crystal system & triclinic & monoclinic & orthorhombic \\
\hline space group & $P-1$ & $P 2{ }_{1} / c$ & $P b c a$ \\
\hline$a / \AA$ & $15.5117(3)$ & $25.5604(3)$ & $16.16337(11)$ \\
\hline$b / \AA$ & $23.6419(5)$ & $13.07217(14)$ & 21.95399(15) \\
\hline$c / \AA$ & $26.1320(6)$ & $24.3250(2)$ & 23.91722(19) \\
\hline$\alpha /^{\circ}$ & $70.130(2)$ & & \\
\hline$\beta /^{\circ}$ & $82.8010(19)$ & $103.0541(10)$ & \\
\hline$\gamma /{ }^{\circ}$ & $88.9118(16)$ & & \\
\hline$V / \AA^{3}$ & $8938.9(4)$ & 7917.68(14) & $8487.03(10)$ \\
\hline$Z$ & 4 & 4 & 8 \\
\hline$M_{\mathrm{r}}$ & 1486.69 & 1429.51 & 751.79 \\
\hline$F_{000}$ & 3340 & 3071 & 3248 \\
\hline$d_{\mathrm{c}} / \mathrm{Mg} \cdot \mathrm{m}^{-3}$ & 1.105 & 1.199 & 1.177 \\
\hline$\mu / \mathrm{mm}^{-1}$ & 3.886 & 4.565 & 3.781 \\
\hline max., min. transmission factors & $1.000,0.844^{\mathrm{a}}$ & $1.000,0.528^{b}$ & $1.000,0.549^{b}$ \\
\hline $\mathrm{X}$-radiation, $\lambda / \AA$ & $\mathrm{Cu}-K_{\alpha}, 1.54184$ & $\mathrm{Cu}-K_{\alpha}, 1.54184$ & $\mathrm{Cu}-K_{\alpha}, 1.54184$ \\
\hline data collect. temperat. /K & $120(1)$ & $120(1)$ & $120(1)$ \\
\hline$\theta$ range $l^{\circ}$ & 2.2 to 67.3 & 3.5 to 71.6 & 3.7 to 70.9 \\
\hline index ranges $h, k, l$ & $\pm 18, \pm 28,-30 \ldots 31$ & $\pm 31,-14 \ldots 15, \pm 29$ & $\pm 19, \pm 26,-29 \ldots 28$ \\
\hline reflections measured & 194214 & 290294 & 275231 \\
\hline unique $\left[R_{\mathrm{int}}\right]$ & $31939[0.141]$ & $15176[0.079]$ & $8161[0.069]$ \\
\hline observed $[I \geq 2 \sigma(I)]$ & 18256 & 11865 & 7139 \\
\hline phasing & charge flip & charge flip & vld \\
\hline data / restraints / parameters & $31939 / 740 / 1910$ & $15176 / 325$ / 870 & $8161 / 15$ / 469 \\
\hline GooF on $F^{2}$ & 1.018 & 1.022 & 1.035 \\
\hline$R$ indices $[F>4 \sigma(F)] R(F), w R\left(F^{2}\right)$ & $0.0748,0.1731$ & $0.0516,0.1202$ & $0.0353,0.0938$ \\
\hline$R$ indices (all data) $R(F), w R\left(F^{2}\right)$ & $0.1539,0.2065$ & $0.0721,0.1313$ & $0.0431,0.0991$ \\
\hline largest residual peaks $/ \mathrm{e} \cdot \AA^{-3}$ & $1.009,-0.669$ & $1.241,-0.836$ & $0.383,-0.557$ \\
\hline CCDC deposition number & 1967923 & 1967924 & 2051087 \\
\hline
\end{tabular}

${ }^{\mathrm{a}}$ semi-empirical absorption correction; ${ }^{\mathrm{b}}$ numerical absorption correction. 


\section{S7.3 MOLECULAR STRUCTURES}

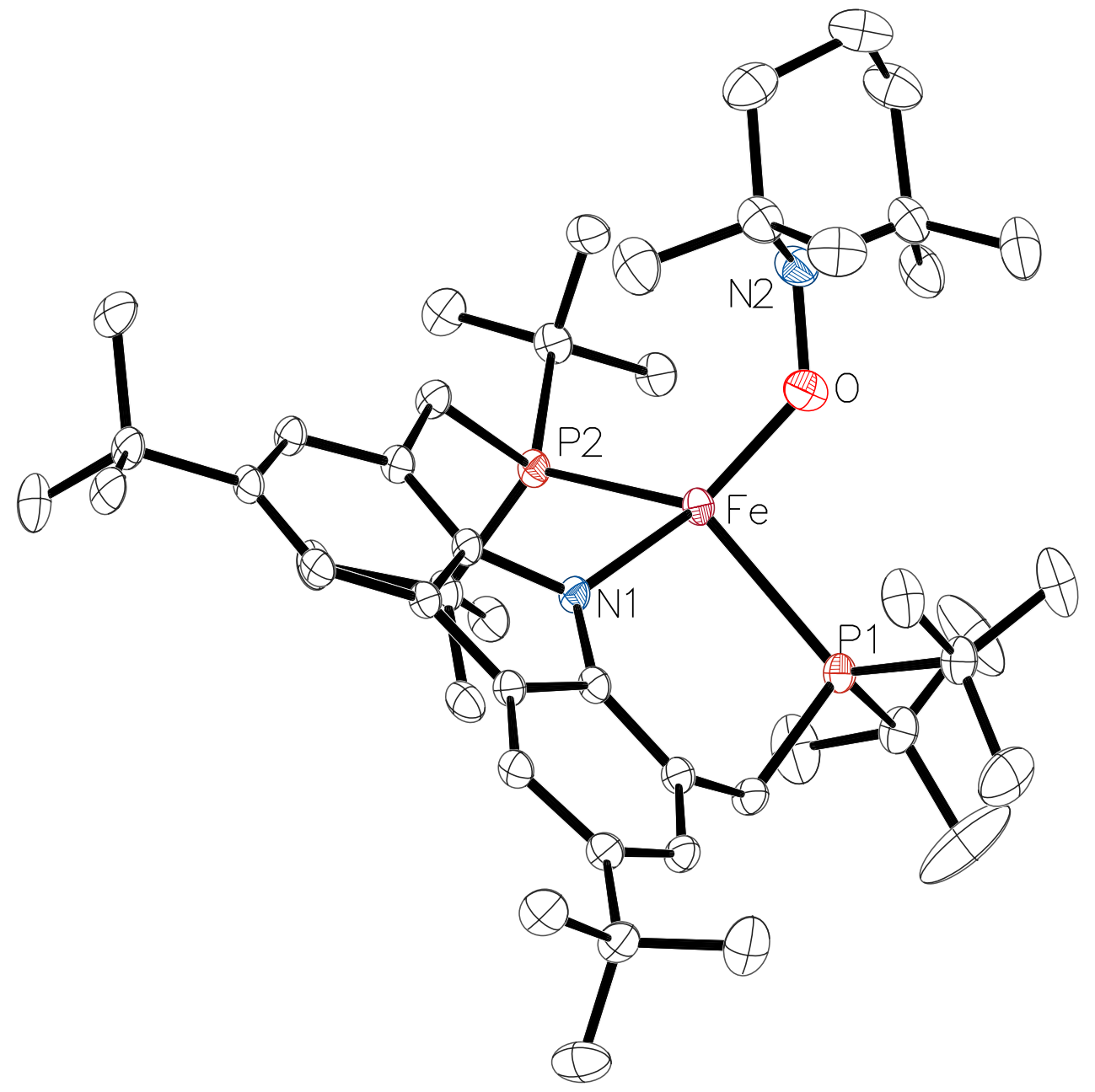

Figure S37. Molecular structure of $\left[{ }^{[\mathrm{Bu}}(\mathrm{PNP}) \mathrm{Fe}(\mathrm{TEMPO})\right](2)$ with displacement ellipsoids drawn at $30 \%$ probability. Hydrogen atoms are omitted for clarity. Selected bond lengths $[\AA \AA]$ and angles [deg]: $\mathrm{Fe}-\mathrm{N} 1$ 2.024(2), Fe-P1 2.4918(7), Fe-P2 2.5771(7), Fe-O 1.872(2), O-N2 1.423(3), P1-Fe-P2 127.37(3), N1Fe-P1 93.91(6), N1-Fe-P2 85.02(6), N1-Fe-O 132.22(9), O-Fe-P1 96.69(7), O-Fe-P2 122.24(7), Fe-ON2 130.13(17). 


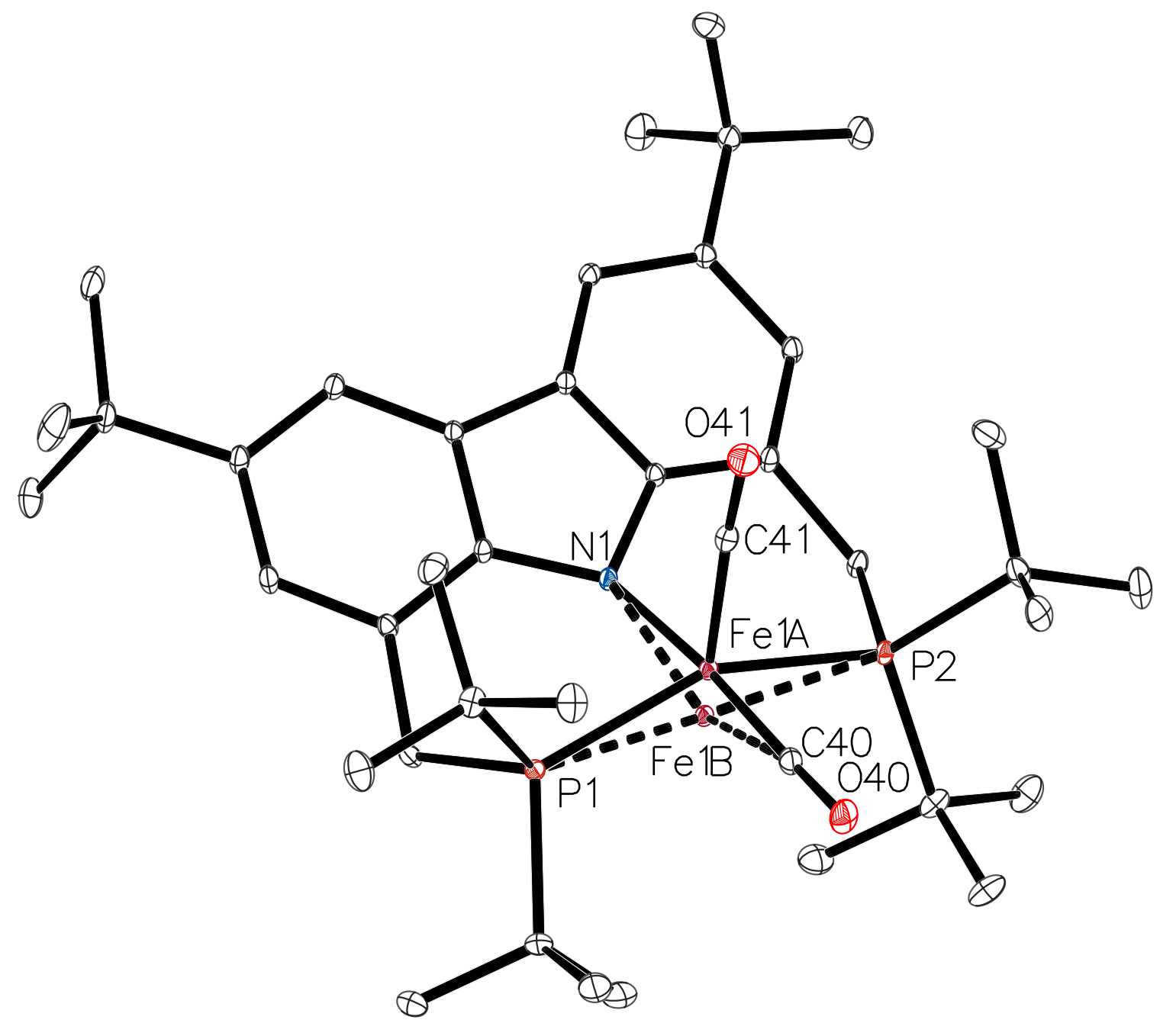

Figure S38. Molecular structure of $\left[{ }^{t B u}(\mathrm{PNP}) \mathrm{Fe}(\mathrm{CO})_{2}\right](3)$ with displacement ellipsoids drawn at $30 \%$ probability. Hydrogen atoms are omitted for clarity. Selected bond lengths $[\AA]$ and angles [deg]: Fe1A-N1 2.0497(12), Fe1B-N1 2.085(5), Fe1A-P1 2.3450(9), Fe1B-P1 2.313(5), Fe1A-P2 2.3220(8), Fe1B-P2 2.261(5), Fe1A-C40 1.7435(14), Fe1B-C40 1.862(5), Fe1A-C41 1.8178(15), C40-O40 1.1602(16), C41O41 1.1460(17), N1-Fe1A-C40 173.86(5), P1-Fe1A-P2 154.896(15), P1-Fe1B-P2 169.7(3), C40-Fe1AC41 90.52(6). 


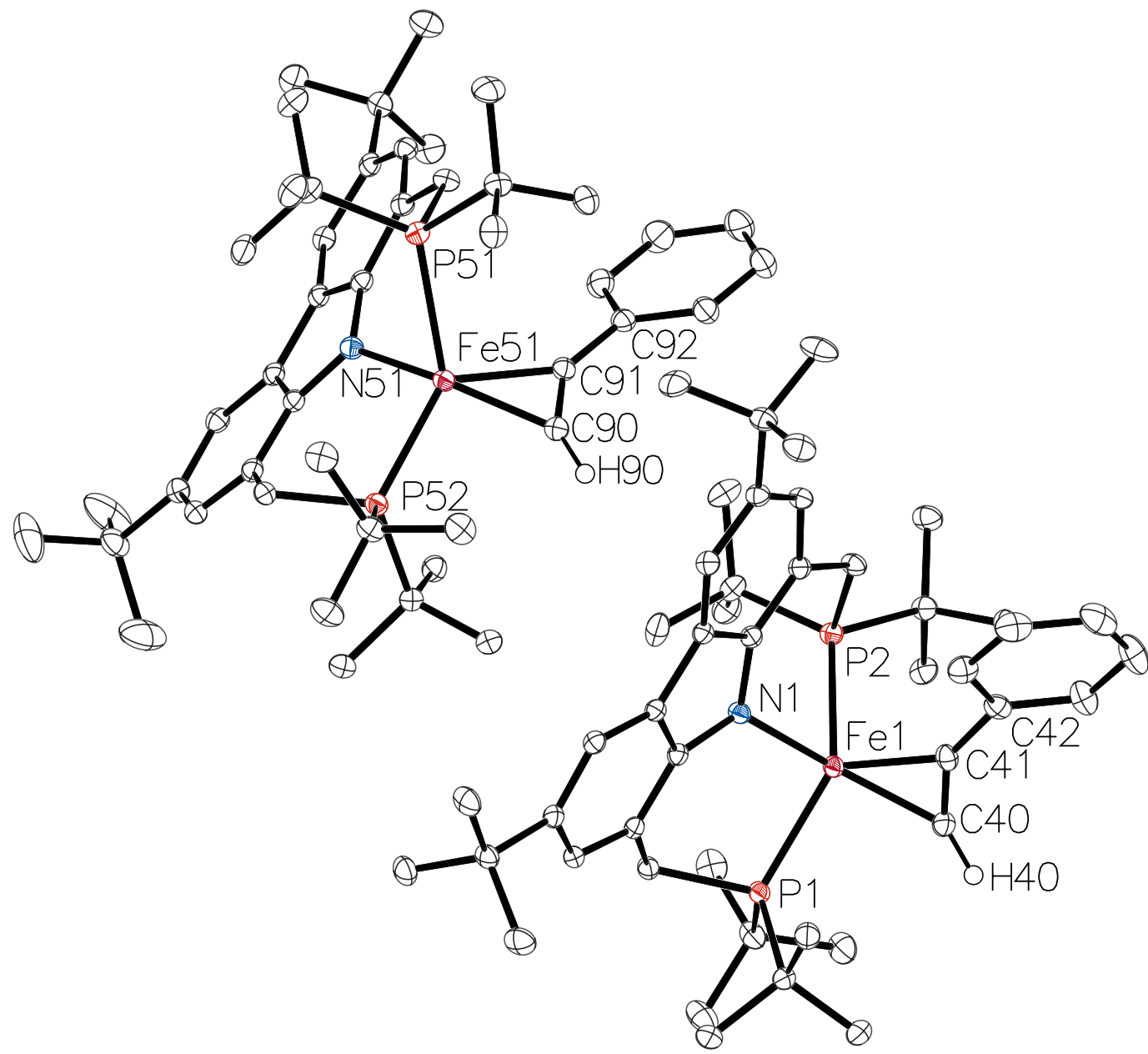

Figure S39. Molecular structure of $\left[{ }^{[\mathrm{Bu}}(\mathrm{PNP}) \mathrm{Fe}(\mathrm{HCCPh})\right](4)$ with displacement ellipsoids drawn at $30 \%$ probability. Hydrogen atoms except $\mathrm{H} 40$ and $\mathrm{H} 90$ are omitted for clarity. Values in squared brackets correspond to second independent molecule. Selected bond lengths $[\AA \AA]$ and angles [deg]: Fe1-N1 1.9817(15) [1.9824(16)], Fe1-P1 2.3593(5) [2.5791(5)], Fe1-P2 2.5662(6) [2.3458(5)], Fe1-C40 1.981(2) [1.978(2)], Fe1-C41 1.9705(19) [1.9759(19)], C40-C41 1.265(3) [1.269(3)], P1-Fe1-P2 126.34(2) [125.21(2)], N1-Fe1-C40 147.75(8) [151.59(8)], N1-Fe1-C41 117.73(7) [122.57(8)], N1-Fe1-P1 94.16(4) [83.16(5)], N1-Fe1-P2 85.11(5) [93.35(5)], P1-Fe1-C40 93.48(6) [115.70(6)], P1-Fe1-C41 126.24(6) [99.73(6)], P2-Fe1-C40 114.71(6) [92.15(6)], P2-Fe1-C41 100.04(6) [126.23(6)], C41-C40H40 144.3(15) [146.0(15)], C40-C41-C42 145.4(2) [144.4(2)]. 


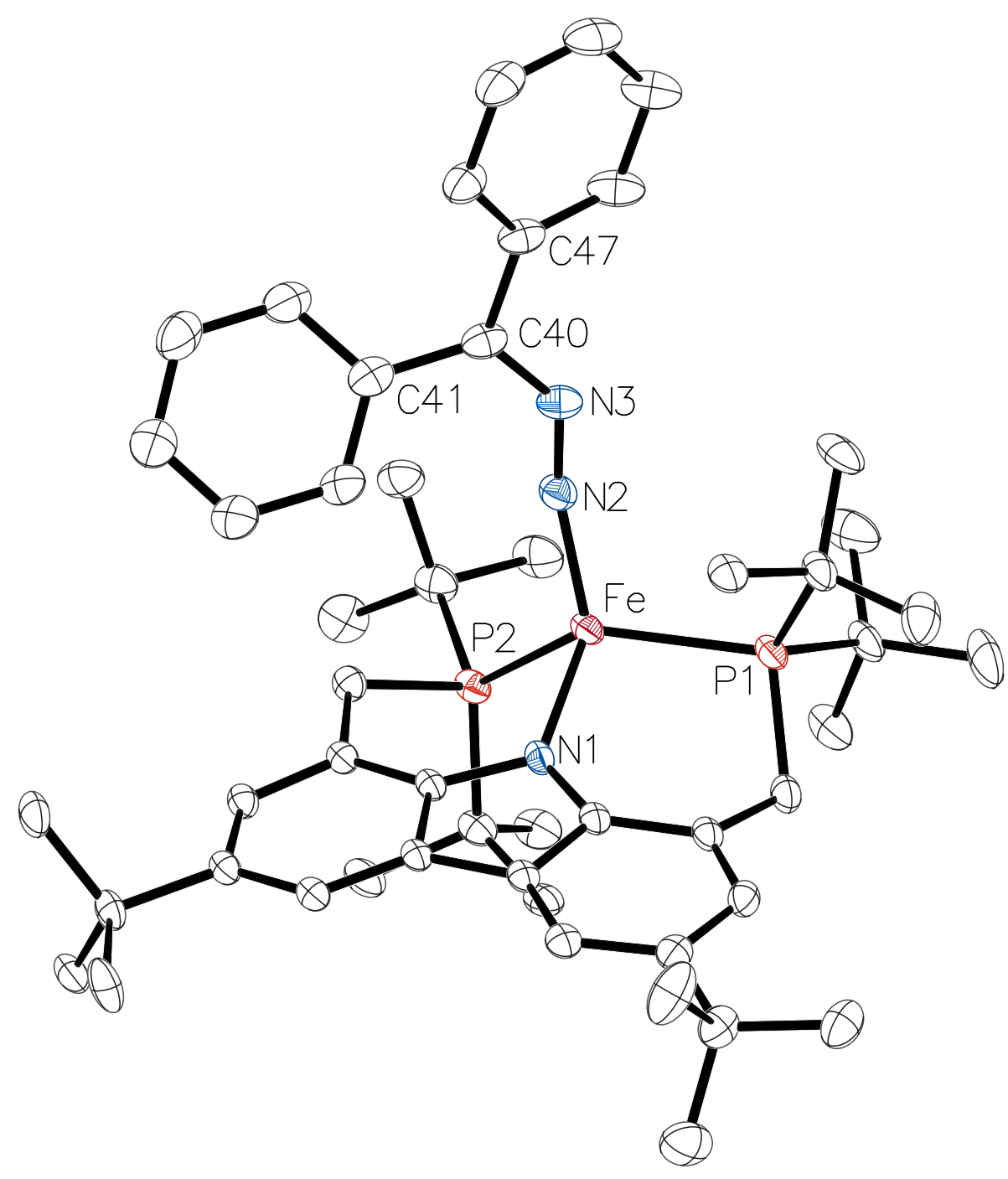

Figure S40. Molecular structure of $\left[{ }^{t \mathrm{Bu}}(\mathrm{PNP}) \mathrm{Fe}\left(\mathrm{N}_{2} \mathrm{CPh}_{2}\right)\right](5)$ with displacement ellipsoids drawn at $30 \%$ probability. Hydrogen atoms are omitted for clarity. Selected bond lengths $[\AA \AA]$ and angles [deg]: $\mathrm{Fe}-\mathrm{N} 1$ 1.969(2), Fe-P1 2.3376(8), Fe-P2 2.4723(8), Fe-N2 1.765(2), N2-N3 1.241(3), N3-C40 1.328(4), C40C41 1.469(4), C40-C47 1.469(4), P1-Fe-P2 132.32(3), N1-Fe-P1 95.19(6), N1-Fe-P2 89.01(6), N1-FeN2 127.13(10), P1-Fe-N2 106.07(8). P2-Fe-N2 108.71(8), Fe-N2-N3 161.2(2), N2-N3-C40 133.7(3), N3-C40-C41 120.7(3), N3-C40-C47 115.9(3). 


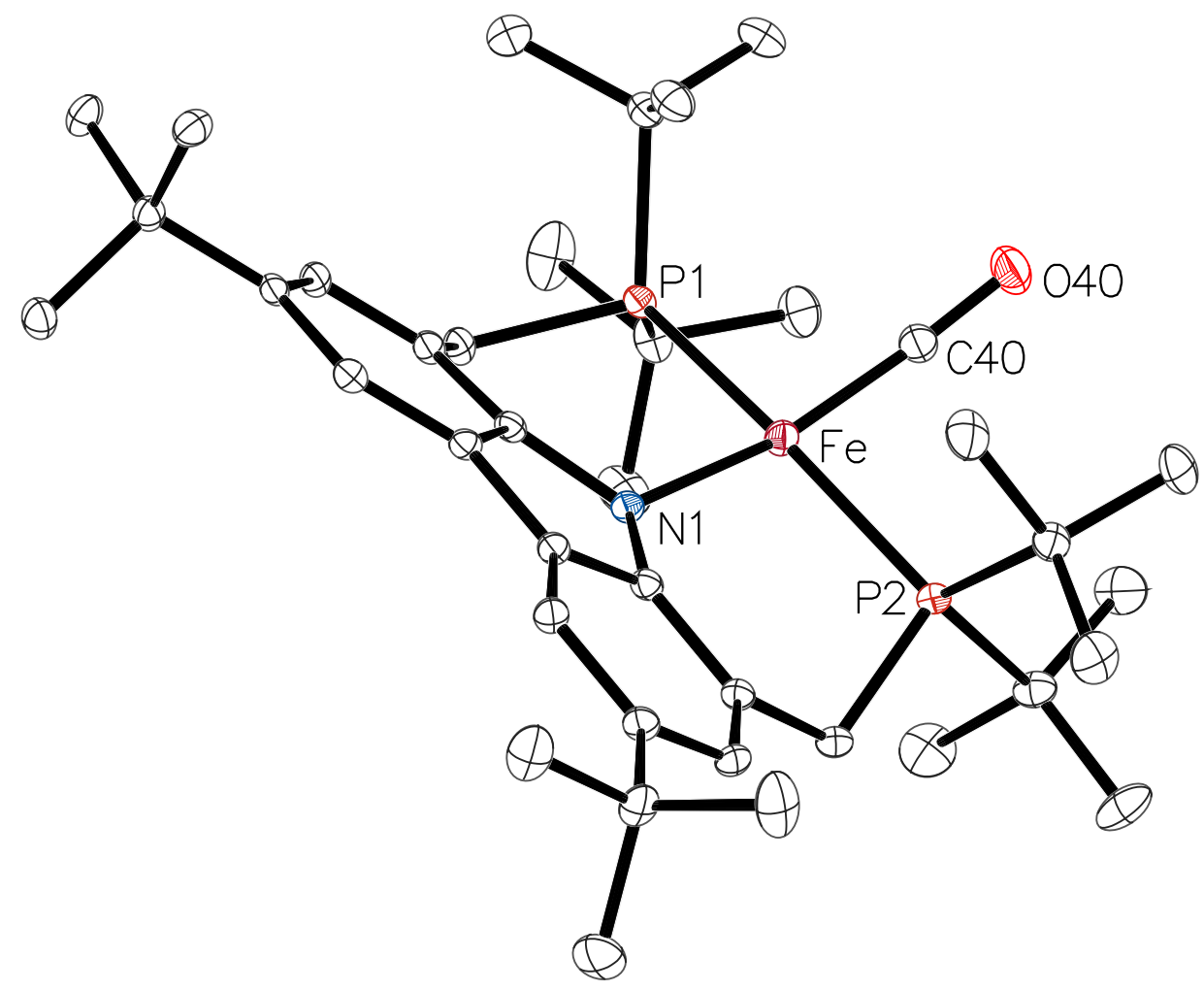

Figure S41. Molecular structure of $\left[{ }^{t \mathrm{Bu}}(\mathrm{PNP}) \mathrm{Fe}(\mathrm{CO})\right](6)$ with displacement ellipsoids drawn at $30 \%$ probability. Hydrogen atoms are omitted for clarity. Selected bond lengths $[\AA]$ and angles [deg]: $\mathrm{Fe}-\mathrm{N} 1$ 2.009(3), Fe-P1 2.2783(9), Fe-P2 2.2830(9), Fe-C40 1.708(4), C40-O40 1.176(4), P1-Fe-P2 166.65(4), N1-Fe-P1 93.26(8), N1-Fe-P2 93.47(8), N1-Fe-C40 161.26(16), C40-Fe-P1 88.84(12), C40-Fe-P2 88.48(12), Fe-C40-O40 173.0(4). 


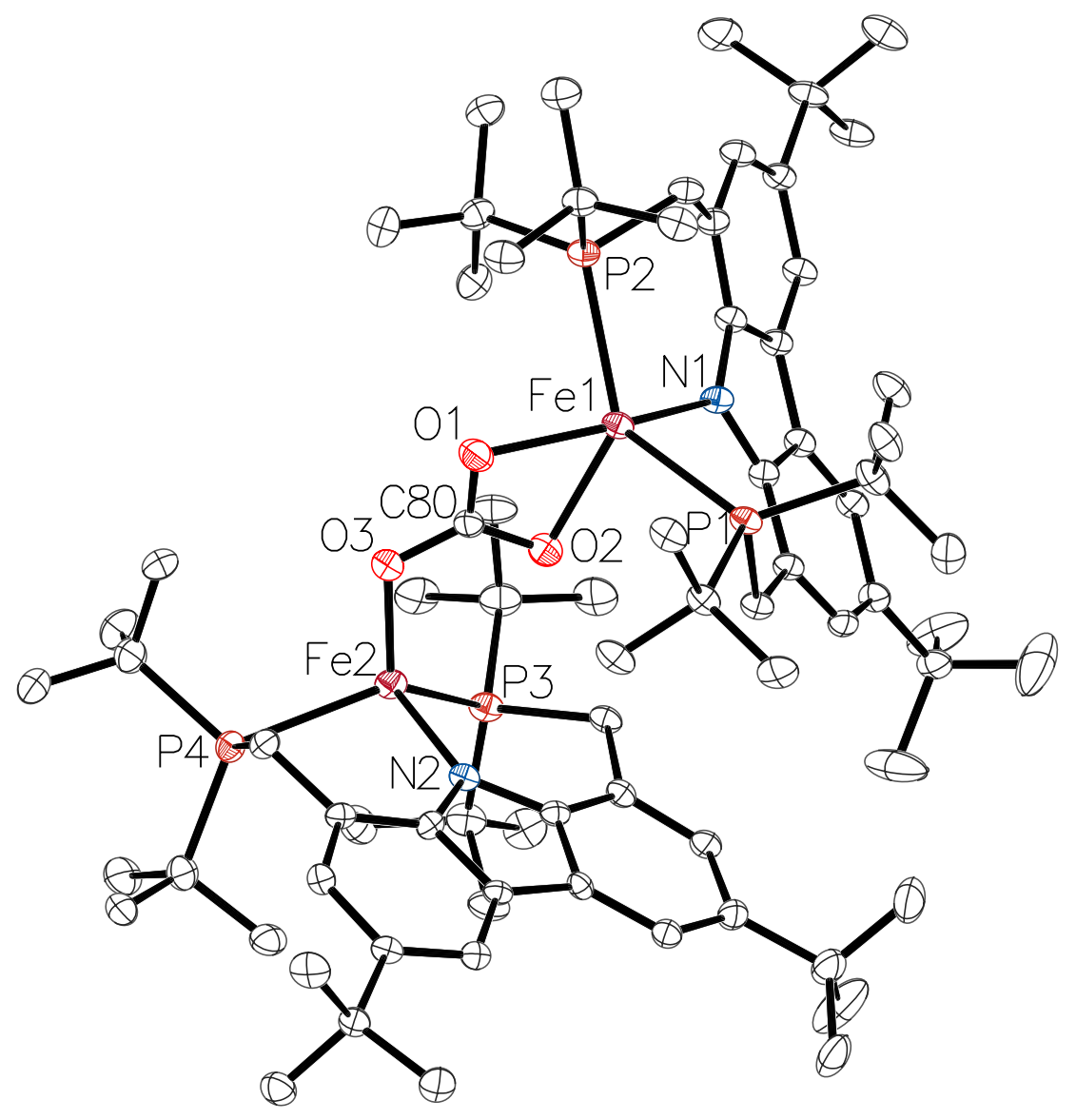

Figure S42. Molecular structure of $\left.\left[{ }^{t \mathrm{Bu}}(\mathrm{PNP}) \mathrm{Fe}\right)_{2}\left(\mathrm{CO}_{3}\right)\right](7)$ with displacement ellipsoids drawn at $30 \%$ probability. Hydrogen atoms are omitted for clarity. Selected bond lengths $[\AA]$ and angles [deg]: Fe1-N1 2.018(4), Fe1-P1 2.4950(15), Fe1-P2 2.4548(15), Fe1-O1 2.109(4), Fe1-O2 2.173(4), Fe2-N2 1.991(4), Fe2-P3 2.4450(16), Fe2-P4 2.4902(16), Fe2-O3 1.953(4), O1-C80 1.276(6), O2-C80 1.279(6), O3-C80 1.292(6), P1-Fe1-P2 127.20(5), N1-Fe1-O1 151.92(17), N1-Fe1-P1 85.90(12), N1-Fe1-P2 92.31(12), N1-Fe1-O2 100.51(15), O1-Fe1-P1 115.29(12), O1-Fe1-P2 88.90(11), O1-Fe1-O2 61.42(14), P3-Fe2P4 131.78(6), N2-Fe2-O3 105.32(16), N2-Fe2-P3 95.88(12), N2-Fe2-P4 94.64(12), O3-Fe2-P3 124.37(12), O3-Fe2-P4 97.47(12), O1-C80-O2 117.8(5), O1-C80-O3 121.6(5), O2-C80-O3 120.6(5). 


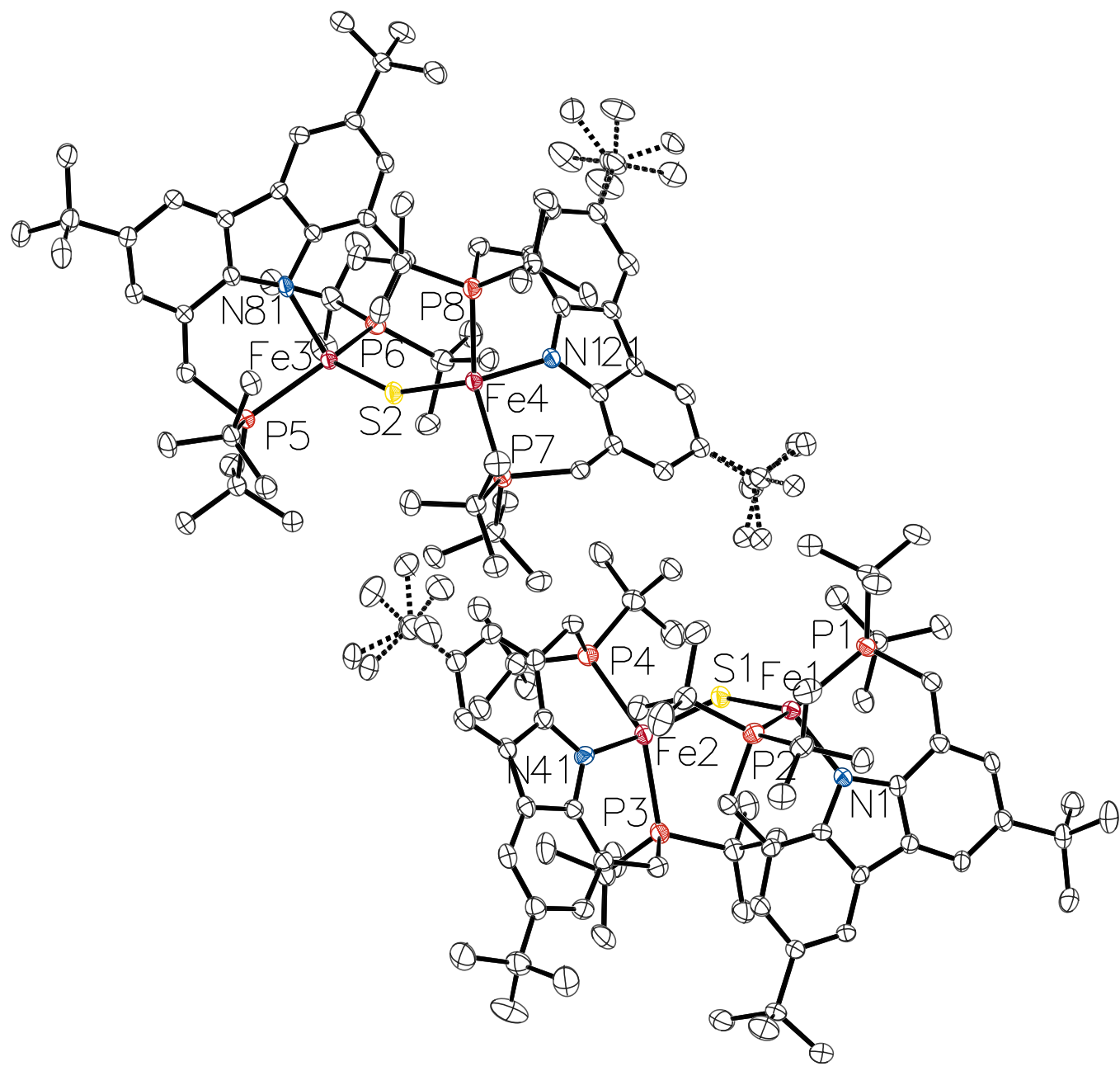

Figure S43. Molecular structure of $\left.\left[{ }^{t \mathrm{Bu}}(\mathrm{PNP}) \mathrm{Fe}\right)_{2} \mathrm{~S}\right](\mathbf{8})$ with displacement ellipsoids drawn at $30 \%$ probability. Hydrogen atoms are omitted for clarity. Values in squared brackets correspond to second independent molecule. Selected bond lengths [Å] and angles [deg]: Fe1-N1 1.986(4) [1.981(4)], Fe1-P1 2.4022(17) [2.4100(16)], Fe1-P2 2.4982(18) [2.4795(17)], Fe1-S1 2.1961(17) [2.1925(16)], Fe2-N41 1.978(4) [1.985(4)], Fe2-P3 2.4380(18) [2.3963(16)], Fe2-P4 2.4308(17) [2.4928(18)], Fe2-S1 2.2113(17) [2.1897(16)], P1-Fe1-P2 130.90(7) [133.03(6)], N1-Fe1-S1 130.15(15) [130.76(14)], N1-Fe1-P1 94.02(13) [94.91(13)], N1-Fe1-P2 86.35(14) [86.35(13)], P3-Fe2-P4 132.53(7) [133.50(6)], N41-Fe2-S1 112.44(15) [130.22(15)], N41-Fe2-P3 95.01(14) [94.74(13)], N41-Fe2-P4 93.72(13) [86.31(14)], Fe1S1-Fe2 145.87(8) [150.16(8)]. 


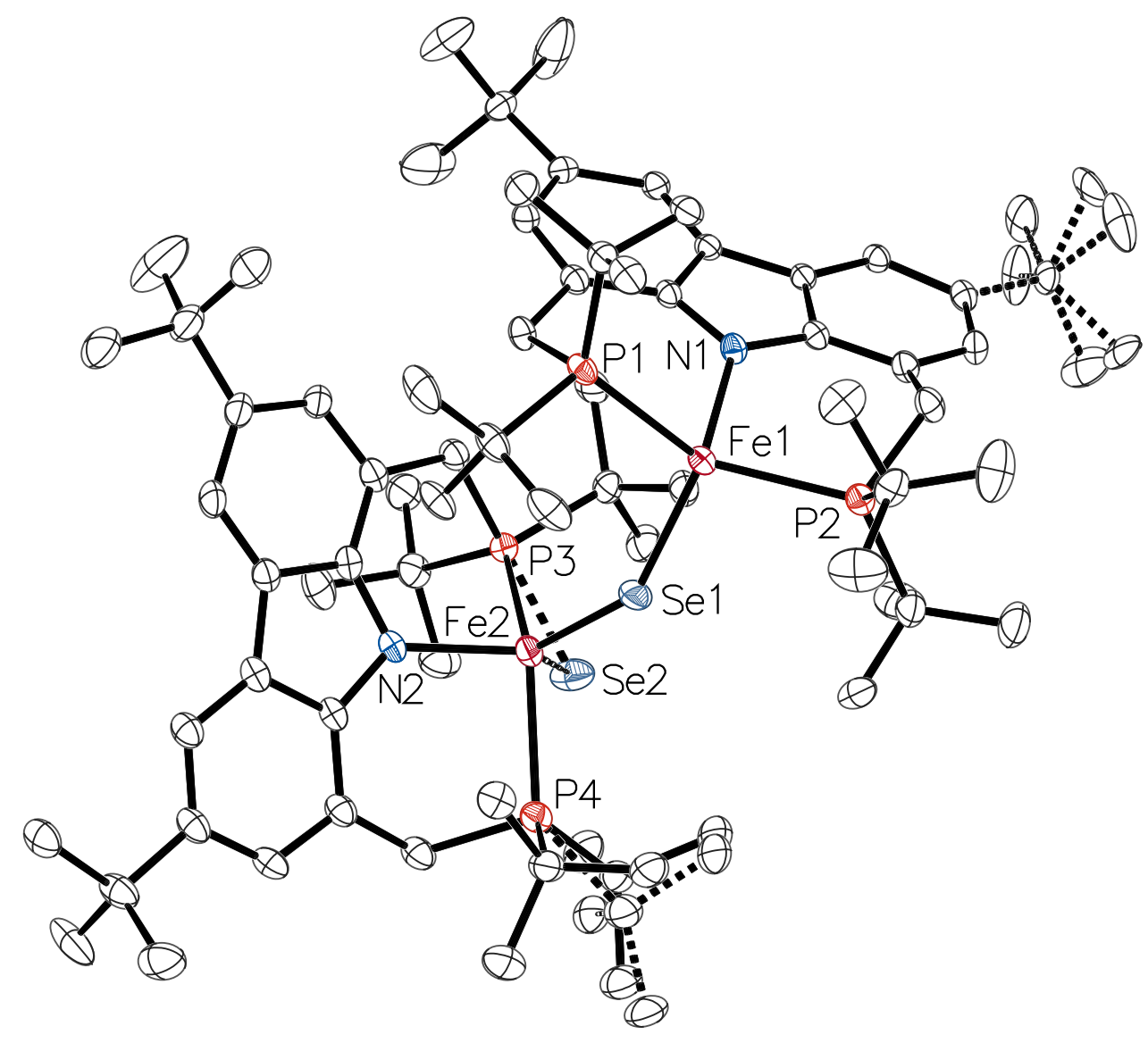

Figure S44. Molecular structure of $\left[\left(^{t \mathrm{Bu}}(\mathrm{PNP}) \mathrm{Fe}\right)_{2} \mathrm{Se}\right](\mathbf{9})$ with displacement ellipsoids drawn at $30 \%$ probability. Hydrogen atoms are omitted for clarity. Selected bond lengths $[\AA]$ and angles [deg]: Fe1-N1 1.987(3), Fe1-P1 2.4958(12), Fe1-P2 2.4373(12), Fe1-Se1 2.3277(7), Fe2-N2 1.984(3), Fe2-P3 2.5512(12), Fe2-P4 2.4100(12), Fe2-Se1 2.3375(7), Fe2-Se2 2.303(5), P3-Se2 2.092(5), P1-Fe1-P2 129.59(4), N1-Fe1-Se1 125.42(9), N1-Fe1-P1 89.58(9), N1-Fe1-P2 96.74(9), P3-Fe2-P4 132.14(4), N2Fe2-Se1 125.86(10), N2-Fe2-P3 84.44(10), N2-Fe2-P4 93.81(10), Fe1-Se1-Fe2 143.55(3), P3-Se2-Fe2 70.83(16). 


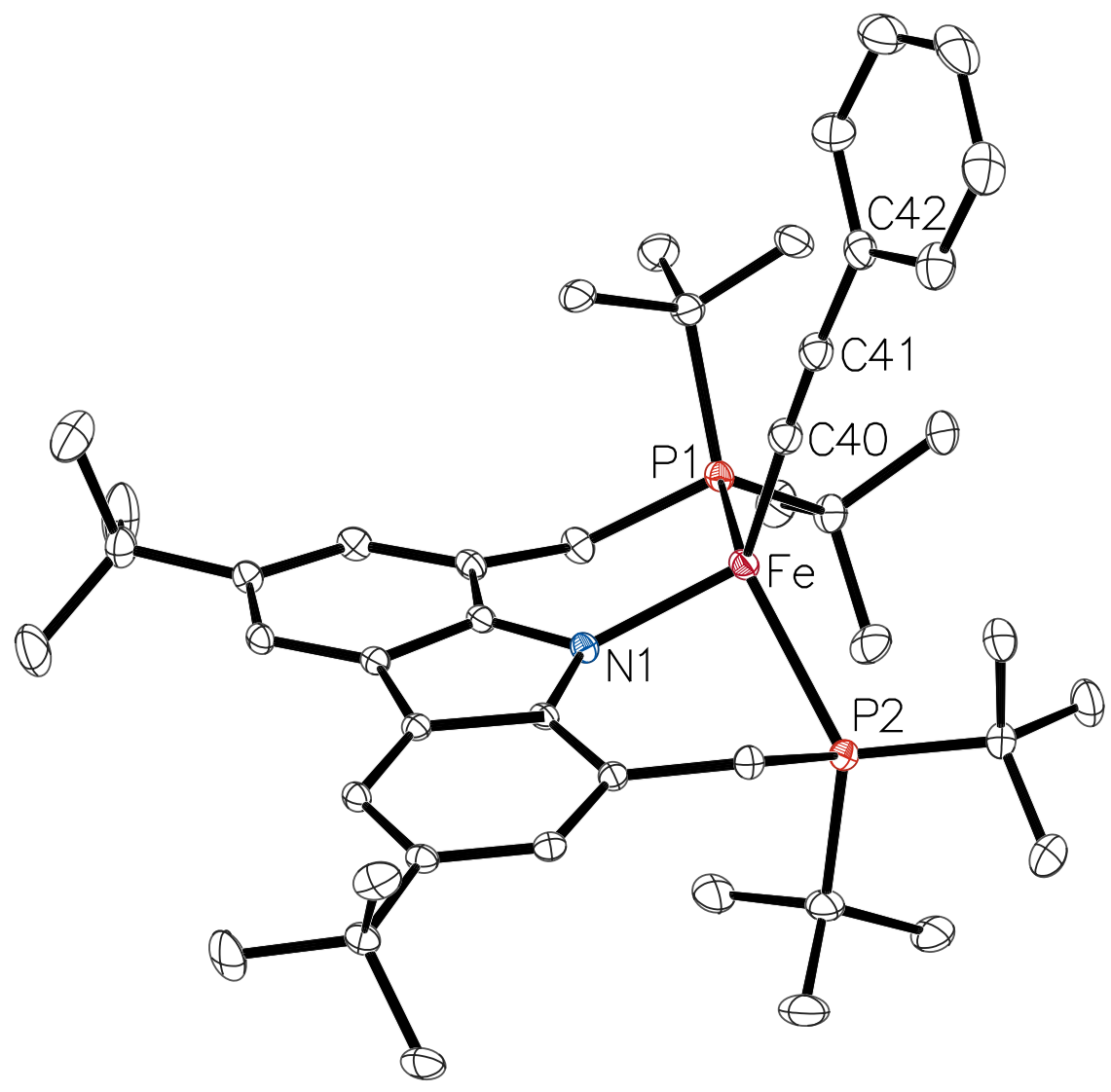

Figure S45. Molecular structure of $\left[{ }^{t \mathrm{Bu}}(\mathrm{PNP}) \mathrm{Fe}(\mathrm{CCPh})\right]$ with displacement ellipsoids drawn at $30 \%$ probability. Hydrogen atoms are omitted for clarity. Selected bond lengths $[\AA \AA]$ and angles [deg]: Fe-N1 1.9856(14), Fe-P1 2.4358(5), Fe-P2 2.4964(5), Fe-C40 2.003(2), C40-C41 1.215(3), C41-C42 1.436(3), P1-Fe-P2 131.518(19), N1-Fe-C40 120.43(7), N1-Fe-P1 96.84(4), N1-Fe-P2 89.55(4), Fe-C40-C41 173.04(18), C40-C41-C42 175.2(2). 


\section{S8 LITERATURE}

(1) Higuchi, J.; Kuriyama, S.; Eizawa, A.; Arashiba, K.; Nakajima, K.; Nishibayashi, Y. Preparation and Reactivity of Iron Complexes Bearing Anionic Carbazole-Based PNPType Pincer Ligands toward Catalytic Nitrogen Fixation. Dalt. Trans. 2018, 47 (4), 11171121.

(2) Kaupp, M.; Bühl, M.; Malkin, V. G. Calculation of NMR and EPR Parameters; WileyVCH: Weinheim, 2004.

(3) Köhler, F. H. Paramagnetic Complexes in Solution: The NMR Approach. In Encyclopedia of Magnetic Resonance; John Wiley \& Sons, Ltd: Chichester, UK, 2011.

(4) Kaupp, M.; Köhler, F. H. Combining NMR Spectroscopy and Quantum Chemistry as Tools to Quantify Spin Density Distributions in Molecular Magnetic Compounds. Coord. Chem. Rev. 2009, 253 (19-20), 2376-2386.

(5) Chilton, N. F.; Anderson, R. P.; Turner, L. D.; Soncini, A.; Murray, K. S. PHI: A Powerful New Program for the Analysis of Anisotropic Monomeric and Exchange-Coupled Polynuclear d- and f-Block Complexes. J. Comput. Chem. 2013, 34 (13), 1164-1175.

(6) Stoll, S.; Schweiger, A. EasySpin, a Comprehensive Software Package for Spectral Simulation and Analysis in EPR. J. Magn. Reson. 2006, 178 (1), 42-55.

(7) Becke, A. D. A New Mixing of Hartree-Fock and Local Density-Functional Theories. J. Chem. Phys. 1993, 98 (2), 1372-1377.

(8) Becke, A. D. Density-Functional Thermochemistry. III. The Role of Exact Exchange. J. Chem. Phys. 1993, 98 (7), 5648-5652.

(9) Schäfer, A.; Huber, C.; Ahlrichs, R. Fully Optimized Contracted Gaussian Basis Sets of Triple Zeta Valence Quality for Atoms Li to Kr. J. Chem. Phys. 1994, 100 (8), 5829-5835.

(10) Weigend, F.; Ahlrichs, R. Balanced Basis Sets of Split Valence, Triple Zeta Valence and Quadruple Zeta Valence Quality for H to Rn: Design and Assessment of Accuracy. Phys. Chem. Chem. Phys. 2005, 7 (18), 3297.

(11) Hehre, W. J.; Ditchfield, R.; Pople, J. A. Self-Consistent Molecular Orbital Methods. XII. Further Extensions of Gaussian-Type Basis Sets for Use in Molecular Orbital Studies of Organic Molecules. J. Chem. Phys. 1972, 56 (5), 2257-2261.

(12) Francl, M. M.; Pietro, W. J.; Hehre, W. J.; Binkley, J. S.; Gordon, M. S.; DeFrees, D. J.; Pople, J. A. Self-Consistent Molecular Orbital Methods. XXIII. A Polarization-Type Basis Set for Second-Row Elements. J. Chem. Phys. 1982, 77 (7), 3654-3665.

(13) Hariharan, P. C.; Pople, J. A. The Influence of Polarization Functions on Molecular Orbital Hydrogenation Energies. Theor. Chim. Acta 1973, 28 (3), 213-222.

(14) Frisch, M. J.; Trucks, G. W.; Schlegel, H. B.; Scuseria, G. E.; Robb, M. A.; Cheeseman, J. R.; Scalmani, G.; Barone, V.; Petersson, G. A.; Nakatsuji, H.; et al. Gaussian 16, Revision C.01. Wallingford CT 2016.

(15) Kirchner, B.; Wennmohs, F.; Ye, S.; Neese, F. Theoretical Bioinorganic Chemistry: The Electronic Structure Makes a Difference. Curr. Opin. Chem. Biol. 2007, 11 (2), 134-141. 
(16) Noodleman, L. Valence Bond Description of Antiferromagnetic Coupling in Transition Metal Dimers. J. Chem. Phys. 1981, 74 (10), 5737-5743.

(17) Kabsch, K. in: Rossmann, M. G.; Arnold, E. (eds.), "International Tables for Crystallography" Vol. F, Ch. 11.3, Kluwer Academic Publishers, Dordrecht, The Netherlands, 2001.

(18) SAINT, Bruker AXS GmbH, Karlsruhe, Germany 1997-2019.

(19) CrysAlisPro, Agilent Technologies UK Ltd., Oxford, UK 2011-2014 and Rigaku Oxford Diffraction, Rigaku Polska Sp.z o.o., Wrocław, Poland 2015-2020.

(20) Blessing, R. H. An Empirical Correction for Absorption Anisotropy. Acta Cryst. 1995, A51, $33-38$.

(21) (a) Sheldrick, G. M. SADABS, Bruker AXS GmbH, Karlsruhe, Germany 2004-2016; (b) Krause, L.; Herbst-Irmer, R.; Sheldrick, G. M.; Stalke, D. Comparison of Silver and Molybdenum Microfocus X-ray Sources for Single-Crystal Structure Determination. J. Appl. Cryst. 2015, 48, 3-10.

(22) SCALE3 ABSPACK, CrysAlisPro, Agilent Technologies UK Ltd., Oxford, UK 2011-2014 and Rigaku Oxford Diffraction, Rigaku Polska Sp.z o.o., Wrocław, Poland 2015-2020.

(23) Busing, W. R.; Levy, H. A. High-speed Computation of the Absorption Correction for Single-crystal Diffraction Measurements Acta. Acta Cryst. 1957, 10, 180-2.

(24) (a) Palatinus, L. SUPERFLIP, EPF Lausanne, Switzerland and Fyzikální ústav AV ČR, v. v. i., Prague, Czech Republic, 2007-2014; (b) Palatinus, L.; Chapuis, G. SUPERFLIP. A Computer Program for the Solution of Crystal Structures by Charge Flipping in Arbitrary Dimensions. J. Appl. Cryst. 2007, 40, 786-790; (c) Palatinus, L. The Ccharge-Flipping Algorithm in Crystallography. Acta. Cryst. 2013, B69, 1-16.

(25) (a) Beurskens, P. T.; Beurskens, G.; de Gelder, R.; Smits, J. M. M.; Garcia-Granda, S.; Gould, R. O. DIRDIF-2008, Radboud University Nijmegen, The Netherlands, 2008; (b) Beurskens, P. T. in: Sheldrick, G. M.; Krüger, C.; Goddard, R. (eds.), Crystallographic Computing 3, Clarendon Press, Oxford, UK, 1985, p. 216.

(26) (a) Burla, M. C.; Caliandro, R.; Carrozzini, B.; Cascarano, G. L.; Cuocci, C.; Giacovazzo, C.; Mallamo, M.; Mazzone, A.; Polidori, G.; Siliqi, D. SIR2019, CNR IC, Bari, Italy, 2019; (b) Burla, M. C.; Caliandro, R.; Carrozzini, B.; Cascarano, G. L.; Cuocci, C.; Giacovazzo, C.; Mallamo, M.; Mazzone, A.; Polidori, G. Crystal Structure Determination and Refinement via SIR2014. J. Appl. Cryst. 2015, 48, 306-309.

(27) (a) Sheldrick, G. M. SHELXL-20xx, University of Göttingen and Bruker AXS GmbH, Karlsruhe, Germany 2012-2018; (b) Robinson, W.; Sheldrick, G. M. in: Isaaks, N. W.; Taylor, M. R. (eds.) „Crystallographic Computing 4“, Ch. 22, IUCr and Oxford University Press, Oxford, UK, 1988; (c) Sheldrick, G. M. A Short History of SHELX. Acta Cryst. 2008, 
A64, 112-122; (d) Sheldrick, G. M. Crystal structure refinement with SHELXL. Acta Cryst. 2015, $C 71,3-8$.

(28) (a) Rollett, J. S. in: Ahmed, F. R.; Hall, S. R.; Huber, C. P. (eds.) „Crystallographic Computing “ p. 167, Munksgaard, Copenhagen, Denmark, 1970; (b) Watkin, D. in: Isaaks, N. W.; Taylor, M. R. (eds.) „Crystallographic Computing 4“, Ch. 8, IUCr and Oxford University Press, Oxford, UK, 1988; (c) Müller, P.; Herbst-Irmer, R.; Spek, A. L.; Schneider, T. R.; Sawaya, M. R. in: Müller, P. (ed.) "Crystal Structure Refinement”, Ch. 5, Oxford University Press, Oxford, UK, 2006; (d) Watkin, D. Structure Refinement: Some Background Theory and Practical Strategies. J. Appl. Cryst. 2008, 41, 491-522.

(29) Thorn, A.; Dittrich, B.; Sheldrick, G. M. Enhanced Rigid-Bond Restraints. Acta Cryst. 2012, A68, 448-451.

(30) v. d. Sluis, P.; Spek, A. L. BYPASS: An Effective Method for the Refinement of Crystal Structures Containing Disordered Solvent Regions. Acta Cryst. 1990, A46, 194-201; (b) Spek, A. L. PLATON SQUEEZE: A Tool for the Calculation of the Disordered Solvent Contribution to the Calculated Structure Factors. Acta Cryst. 2015, C71, 9-18.

(31) Spek, A. L. PLATON, Utrecht University, The Netherlands; (b) Spek, A. L. Single-Crystal Structure Validation with the Program PLATON. J. Appl. Cryst. 2003, 36, 7-13. 